\title{
River Devices to Recover Energy with Advanced Materials
}

\author{
River DREAM
}

US Department of Energy grant (DE-EE0004571)

May 2013 


\section{TABLE of CONTENTS}

- ABSTRACT

- INTRODUCTION

- EXPERIMENTAL

$>$ Galloping Oscillator

o Describing-function description of fluid-structure interaction:

o A simple model of energy conversion:

$>$ Computational Fluid Dynamics

o Force Coefficients:

$>$ GHEED Generations

o Database Development:

o GHEED Design Evolution:

$>$ Morphing

$>$ Dielectric Elastomer Generator testing

$>$ DEG modeling

- RESULTS AND DISCUSSION

$>$ Galloping Oscillator

$>$ Computational Fluid Dynamics

$>$ GHEED Generations

o Database Development:

o GHEED Design Evolution:

$>$ Morphing

$>$ DEG Testing

o Single DEG Simulation and Calibration to Data:

o Coupled DEG Simulation:

$>$ DEG Modeling

o Two-phase DEG Model and Best Fit Parameters:

o Scaling Equations:

o Combined System Modeling

o Coupling a DEG to Hydrodynamic Model of Galloping Bluff Body:

o Sizing a 1 Kilowatt Demonstration Unit:

- LESSONS LEARNED

$>$ Galloping Body

o Future Experiments:

o Other Structural Oscillation Modes:

$>$ Shape Morphing

$>$ DEG

$>$ Total System

$>$ Larger Prototype

- COST REDUCTION PATHWAYS

- CONCLUSION 


\section{ABSTRACT}

This report describes the development and testing of a Galloping Hydro Electric Energy Device (GHEED) which is intended to utilize a Dielectric Elastomer Generator (DEG). The mechanical side of the GHEED includes a shape morphing element, and has been designed to convert the kinetic energy of low head and/or turbulent flows into repeating, oscillating motion. The intent is to couple this motion to a DEG, which will convert the oscillating motion into electrical energy. In this exploratory program, the experimental elements of the mechanical and electrical sides of the program were conducted in parallel; a unifying model has been created to project the potential for a combined device. The mechanical side of the GHEED was developed at the University of Pittsburgh and included: GHEED prototype design and construction, design and implementation of performance enhancing shape morphing elements, and computational fluid dynamics studies facilitating creation of the unifying model. Also described is the testing of a dielectric elastomer generator developed by Stanford Research Institute (SRI), and calibration of a model to data from that generator. In modeling, it was found that the spring rate of a dielectric elastomer generator could be linearized by the addition of an opposed mechanical phase. This arrangement simplifies analysis, and so this additional configuration was modeled. Analysis also showed that viscoelastic losses in the acrylic dielectric used in the SRI generator were undesirably large, and so additional material tests on an alternative silicone dielectric were undertaken at Artificial Muscle Inc. Generator performance based on this new material was also modeled, and the simulation was coupled to a model of a small galloping bluff body in hydrokinetic flow, calibrated to flume data. The representation was then used to size a dielectric elastomer generator suitable for a 1 kilowatt demonstration generator.

\section{INTRODUCTION}

The United States is populated with a vast network of bodies of water including lakes, ponds, rivers and streams. A published study $\{\mathrm{Hall}\}$ was conducted to evaluate the water energy potential of the stream segment portion throughout the nation to determine what really may be available from this power generation source. This study found that the total available potential power is estimated to be $170,000 \mathrm{MW}$, and the low head/low power potential makes up $12 \%$ (21,000 $\mathrm{MW})$ of the total. These resources presently lack a technology to effectively harvest their energy, which is the basis for the River DREAM project we undertook. An essential feature of the project is that the energy generation concept being explored is low profile, largely non-invasive and is expected to leave rivers useable, aesthetically pleasing, and ecologically viable.

The generator we propose to develop is termed a Galloping Hydroelectric Energy Extraction Device (GHEED) which uses a galloping prism to convert kinetic energy from flowing water into linear motion electrical power. The energy is converted into electricity via a dielectric elastomer generator (DEG) which is combined with the galloping mechanism to create a system to effectively generate electricity. 
A phenomenon called galloping has been observed in electrical power lines in the presence of ice and/or rain. Wind blowing across ice-covered power lines creates lift causing the lines to oscillate. Galloping occurs when these oscillations increase to a sustained limit cycle where the oscillations are consistent and powerful. For power lines it is desirable to limit these oscillations but we propose to exploit them to create oscillations in a rigid prism mounted on an elastic support. Through proper design of prism shape, dynamic interaction with the flowing water will be controlled to create a galloping oscillator. This control will be further enhanced by controlling the shape of the prism. The prism will be designed such that its shape changes with the amount of water flow, ensuring that the proper conditions are maintained to encourage the galloping behavior.

The controlled oscillation of the galloping device will be combined with a DEG which was chosen because it is well suited to converting linear motion into electricity. For energy generation the DEG acts as an elastic capacitor. It will be charged while in a strained state, i.e., when the opposing electrodes have maximum surface area and minimum separation distance. Once charged, the linear motion of the oscillator will allow the DEG to relax, reducing the surface area and increasing the separation of the electrodes. This action does work on the charges by separating unlike charges and concentrating like charges. The excess energy is harvested from the DEG in its relaxed state. The linear motion of the oscillator once again strains the DEG and the cycle begins again.

The project has three research objectives:

1. Oscillator development and design

2. DEG characterization and modeling

3. GHEED system modeling and integration

Accomplishing these three objectives will result in the creation of a model that can be used to fully define the operating parameters and performance capabilities of a generator based on the GHEED design. This information will be used in the next phase of product development, the creation of an integrated laboratory scale generator to confirm model predictions.

\section{EXPERIMENTAL}

This section describes the methodologies employed in six key, but separate GHEED development areas; the section concludes with description of the methodologies employed in a seventh development area intended to merge the separate efforts into a unifying projection. 


\section{Galloping Oscillator}

In this research, we are interested in the fluid-structure interaction of certain bluff body shapes, typically right prism shapes that we will refer to as prisms. For certain prism shapes fluid-elastic phenomenon known as galloping can occur; see Figure: A; gallop below:

$L$
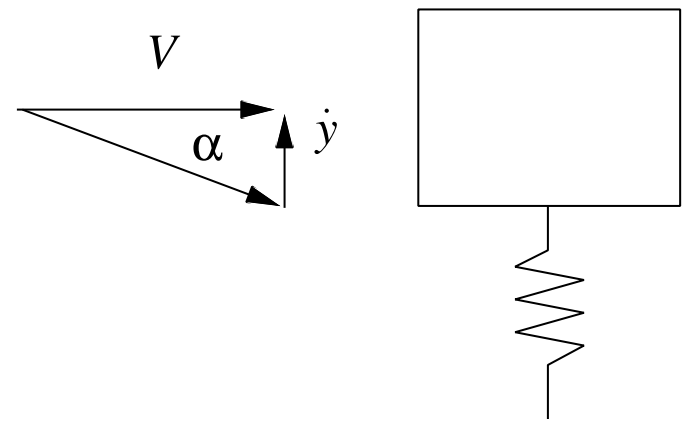

Figure A - Gallop

The oscillation of the device is a single mode. The hydrodynamic interaction with the device occurs through the lift

$$
L(\alpha)=q S C L(\alpha)
$$

where the coefficient of lift may be a non-linear function of the angle of attack

$$
\alpha=\frac{\dot{y}}{V}
$$

The structural transfer function relating force $f$ and velocity $\dot{y}$ is

$$
\frac{y}{f}=G(s)=\frac{s}{m s^{2}+c_{m} s+k}
$$

The force $f$ has two contributions: one is from an equivalent damper that describes the harvesting of energy, the other is from the lift resulting from flow across the device:

$$
f=f_{a}-f_{e}=q S C_{L}(\alpha)-c \dot{y}
$$

The interaction of the hydrodynamics and structural dynamics is a feedback connection as shown in Figure B; feedback below: 


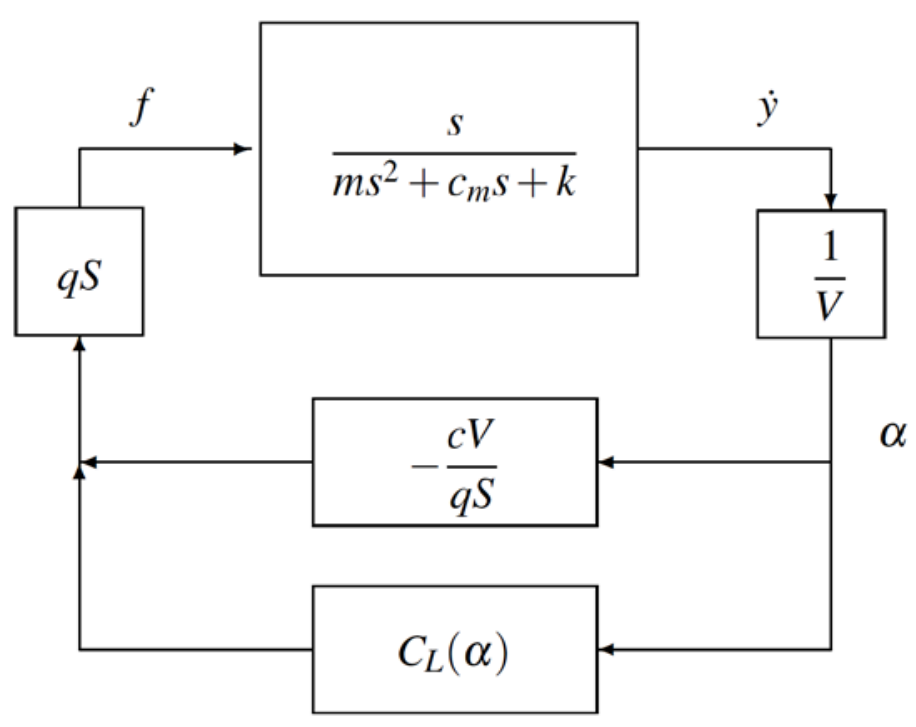

Figure B - Feedback

Analyzing the motion of the device including non-linear effects of the lift is difficult, and often can only be accomplished using numerical simulations. However, we can use the technique of describing functions (also called harmonic balance) to determine characteristics of the oscillation.

\section{Describing-function description of fluid-structure interaction:}

The coefficient of lift, $C_{L}(\alpha)$, is an odd function of $\alpha$ due to symmetry, which can be expanded as

$$
C_{L}(\alpha)=a_{1} \alpha+a_{3} \alpha^{3}+a_{5} \alpha^{5}+a_{7} \alpha^{7}
$$

including enough terms to characterize the changes in curvature over the operating range of interest. Figure C, Coefficient of lift below shows $C_{L}(\alpha)$ for a square prism. 


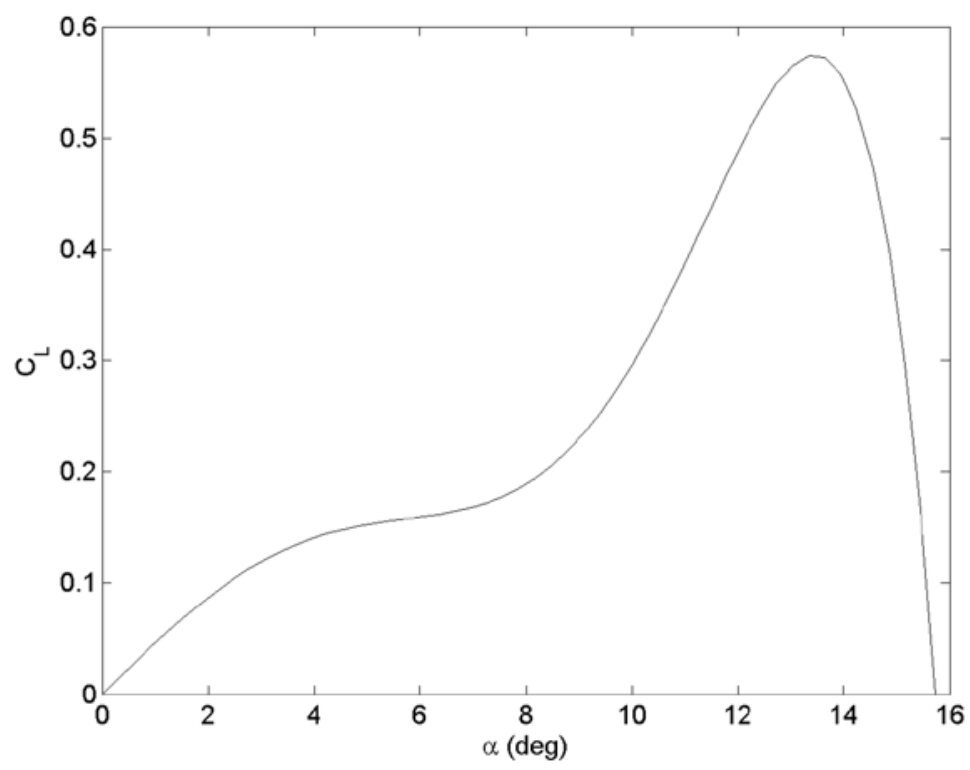

Figure C - Coefficient of Lift

Consider the device to be undergoing sinusoidal motion at frequency $\omega$, so that

$$
\alpha(t)=A \sin (\omega t)=\frac{\omega Y}{V} \sin (\omega t) .
$$

The describing function from $\alpha$ to $f /(q S)$, the feedback path in Figure $B$ is

where

$$
N(\bar{Y}, \beta)=\left(a_{1}-\beta\right)+\frac{3}{4} a_{3} \bar{Y}^{2}+\frac{5}{8} a_{5} \bar{Y}^{4}+\frac{35}{64} a_{5} \bar{Y}^{6}
$$

$$
\beta=\frac{c V}{q S} \text { and } \bar{Y}=\frac{\omega Y}{V}
$$

The stability of the aero elastic system is determined by the characteristic equation

$$
1-N(\bar{Y}, \beta) G(j \omega)=0
$$

and the system will oscillate at $\omega=\omega_{n}=\sqrt{\mathrm{k} / \mathrm{m}}$.

Stability about equilibrium is determined by the linear term (note that $a_{1}=C_{L \alpha}$ ), where for the condition,

$$
C_{L \alpha}=a_{1}>\beta=\frac{c V}{q S}
$$

the linear term of $N(\bar{Y}, \beta)$ is positive and the equilibrium point is unstable. The system response will grow; however, it does not grow without bound since the lift falls off at high angles of attack (the device stalls), and the motion may reach a steady limit cycle oscillation.

The roots of $N(\bar{Y}, \beta)=0$ determine those amplitudes at which the fluid-elastic system operates. The coefficients of the equation $N(\bar{Y}, \beta)=0$ are functions of $\beta$ (that is, functions of the free stream velocity $V$ and the equivalent damping coefficient $c$.) Figure D; Ymax-vs.-beta shows the magnitude of the limit-cycle oscillation for a square prism. 


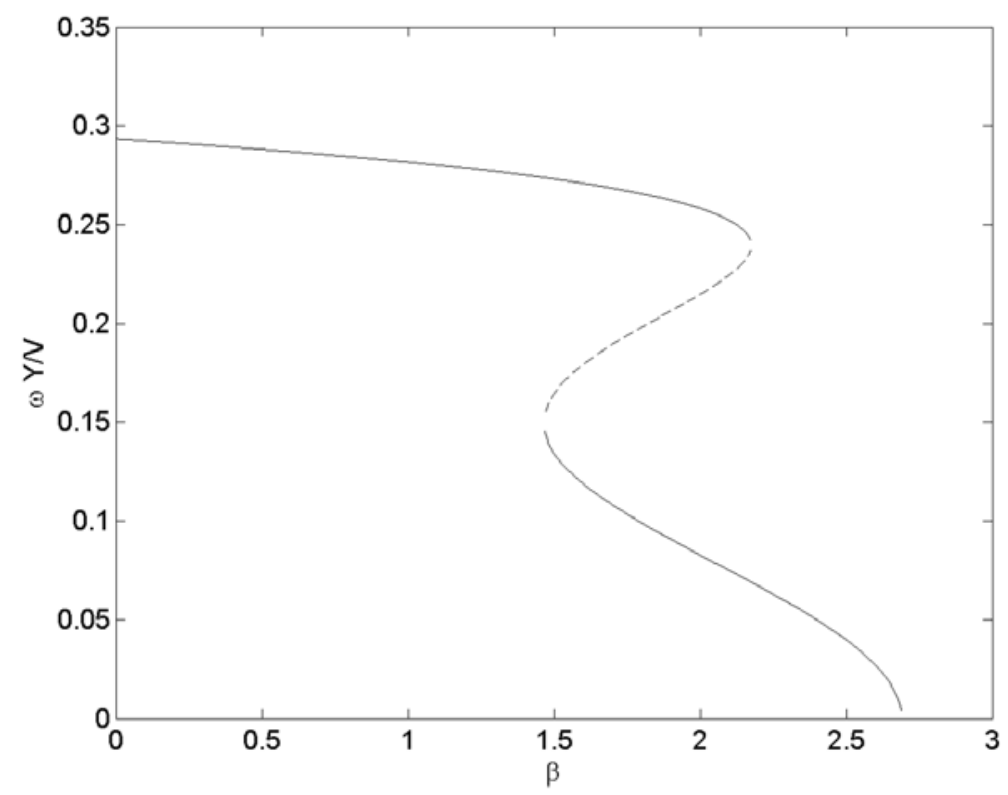

Figure D - Ymax vs. Beta

Note that the magnitude of the limit-cycle oscillation is relatively insensitive to the value of $\beta$ until it nears the critical value of $\beta_{\text {crit }}=a_{1}=2.69$ at which point the device is stabilized by the power generation process.

\section{A simple model of energy conversion:}

The conversion of mechanical energy to electrical energy is made using a generalized transformer with the transformation of force/velocity, $f$ and $\dot{y}$, to voltage/current, $v$ and $i$, described by

$$
f=\theta v \text { and } i=\theta \dot{y}
$$

where $O$ is the electromechanical coupling coefficient. This model describes a large variety of piezoelectric materials. The storage or distribution of electrical energy can most simply be modeled as a resistive load;

$$
v=R i
$$

When combined with the gyrator equations the force/velocity relationship seen from the mechanical side is

which is an equivalent damper with coefficient

$$
f_{e}=\theta^{2} R \dot{y}
$$

$$
c=\theta^{2} R \text {. }
$$

A similar model can be made for a gyrator, which models electro-mechanical inductance, with the result that the equivalent damper with

$$
c=\frac{\kappa^{2}}{R}
$$

where $\kappa$ is the torque or back-emf constant. Which equivalent damper is used depends upon the transduction mode. From the standpoint of the analysis given here both are equivalent when viewed as an equivalent damper. 
For a structure undergoing sinusoidal motion at frequency $\omega$, so that

$$
y(t)=Y \sin (\omega t)
$$

the average power generated per cycle is the average power "dissipated" by this damper;

$$
P_{\text {gen }}=\frac{1}{2} c \omega^{2} Y^{2} \text {. }
$$

The non-dimensional coefficient of performance is

$$
C_{P}=\frac{P_{\text {gen }}}{q S V}=\frac{\frac{1}{2} c \omega^{2} Y^{2}}{q S V}
$$

where $q=\frac{1}{2} \rho V^{2}$ is the dynamic pressure, and $V$ is the flow velocity. The area $S$ is a characteristic area. From this it appears that the equivalent damper should be made as large as possible; however, it should be realized that the amplitude of vibration, $Y$, depends upon the damper's coefficient and the frequency of excitation.

In order to evaluate the power generated by galloping, the describing function equation can be written in terms of the coefficient of performance:

$$
\frac{35}{64} a_{7} C_{P}^{3}+\frac{5}{16} a_{5} \beta C_{P}^{7}+\frac{35}{64} a_{3} \beta^{2} C_{p}+\frac{1}{8} \beta^{3}\left(a_{1}-\beta\right)=0
$$

The roots of this polynomial determine the power generation, where the locus of roots for the coefficient of performance depend upon $\beta$.

It is interesting to note that a single curve describes the operation of this device for varying load and flow velocity. Figure $\mathrm{E}: C_{P}$-vs.- $\beta$ below shows $C_{P}$ versus $\beta$ for a square prism.

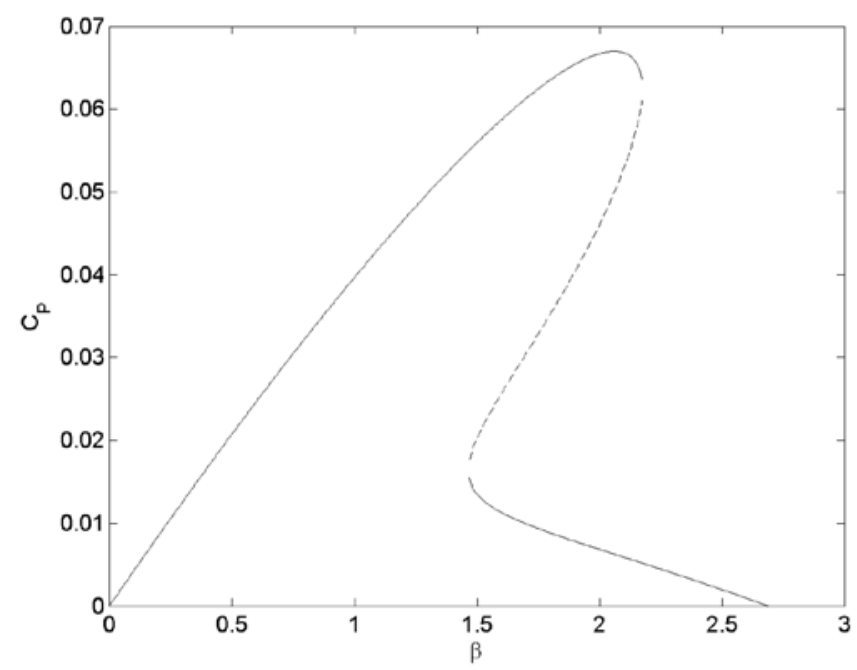

Figure E - Cp vs. Beta

This device is interesting because for certain values of $\beta$, it has multiple stable limitcycles, separated, of course, by unstable limit cycles, where these different limit cycles correspond to the multiple roots of the above polynomial. When considering the use of such a device for hydrokinetic energy harvesting, one would clearly like to operate at a high value of coefficient of performance. 


\section{$\underline{\text { Computational Fluid Dynamics }}$}

For this research, bluff bodies in two-dimensional cross flow are examined. Experimental data sets for the lift force coefficients for both the square \{Parkinson1964\} and the D-shape \{Novak1972\} bluff body geometries exist in the engineering literature; data for other geometries could not be found. These values are used as a standard for comparison to evaluate the predictive ability of a particular Computational Fluid Dynamics (CFD) model. Based upon suggestive results in the flume, trapezoid and equilateral triangle are also considered.

Calculations were performed using double precision. Unless specified otherwise, SIMPLE is employed as the pressure-velocity coupling algorithm. This is a pressurebased segregated algorithm that "uses a relationship between velocity and pressure correction to enforce mass conservation and to obtain the pressure field" \{FLUTHY2010\}. The least-squares cell based method is used for gradient and derivative evaluations in all simulations. The standard pressure discretization is used in all simulations. The momentum equation uses second-order discretization in the earlier simulations. In subsequent simulations, first-order discretization is used for the computational time-savings, as the difference between first and second order does not make a significant difference in result. The turbulent kinetic energy equation, specific dissipation rate equation, and transient formulation all use first order discretization in all simulations. Second order and other temporal schemes are not explored because of computation-time considerations. Under-relaxation factors remain at the default ANSYS settings because convergence is not an issue in any of the simulations. Finally, residuals for all simulations are set to a minimum of $10^{-5}$ (typically $10^{-6}$ ).

There are three boundary conditions:

1. The inlet condition employed is the FLUENT velocity-inlet condition to set specific velocity conditions, turbulent kinetic energy, and specific dissipation rate values for a particular boundary.

2. There are two different outlet conditions used:

a. In earlier simulations, the pressure-outlet condition is used, allowing the user to set a gauge pressure at the boundary and define backflow turbulent kinetic energy and specific dissipation rate values.

b. In later simulations, the outflow condition is used, and is used when the boundary's characteristics are not known; ANSYS extrapolates them from the interior.

3. The wall-boundary condition imposes a no-slip condition on the bluff body surface.

\section{Force Coefficients:}

Square-prism results are displayed with both experimental data and simulation data, thus, including a benchmark to evaluate the result.

Figure $\mathrm{F}$ is the first trial in a series of tests to determine mesh independence. 


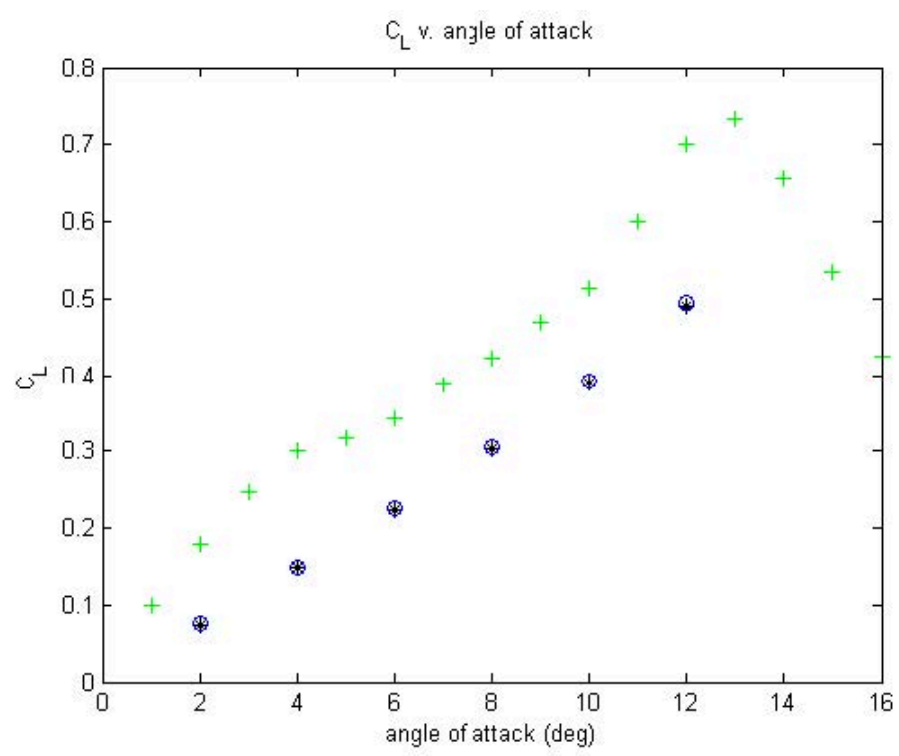

Figure: F - The first in a series of mesh independence tests: 7440 cells.

Lab frame coefficient of lift is the benchmarking value. Conceptually, if a subsequent doubling in detail does not provide significant change in the output, then the mesh is not the primary source of error. As noted in Figure G, there is a change in the output that is less than $10 \%$ compared to the result from Figure F.

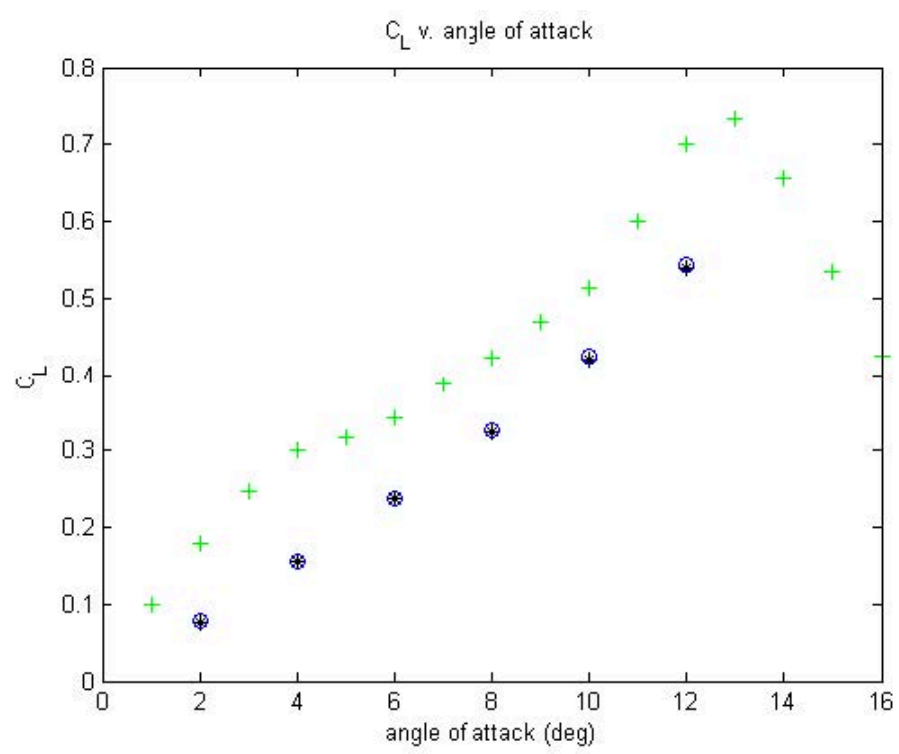

Figure G - The second trial in a series of mesh independence tests: 30000 cells.

In Figure $\mathrm{H}$ the output shifts back to approximately the same as that of what's displayed in Figure F trial. 


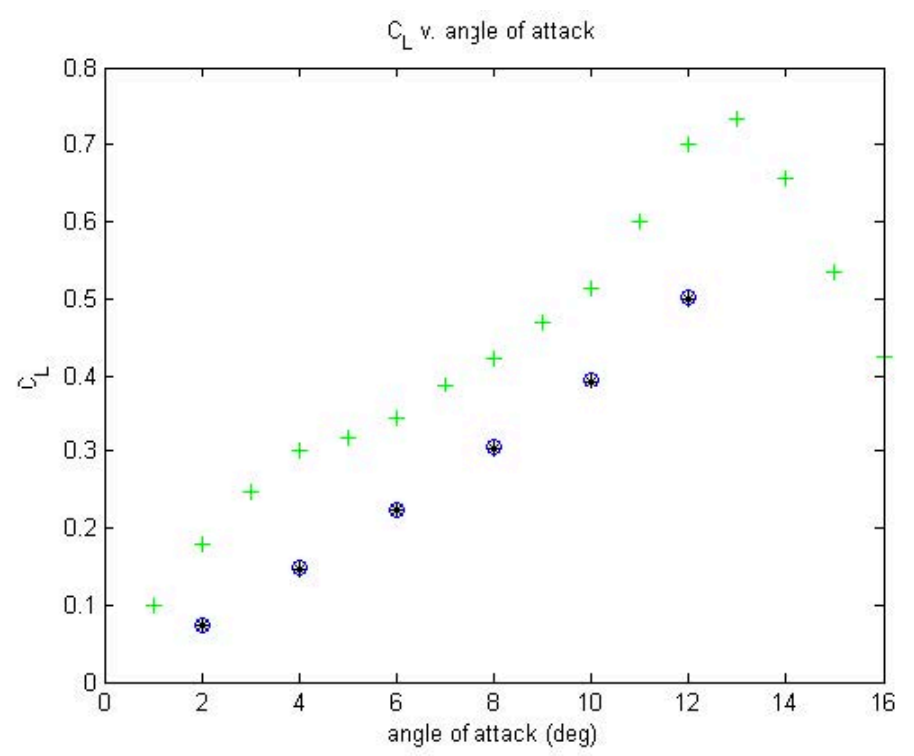

Figure $\mathbf{H}$ - The third trial in a series of mesh independence tests: 118560 cells.

It is also clear that the simulated coefficient of lift has a similar general slope to the experimental data, but the values are consistently too low by a value of 0.1 . The PISO pressure-velocity coupling scheme is also explored. Consider the results in Figure J.

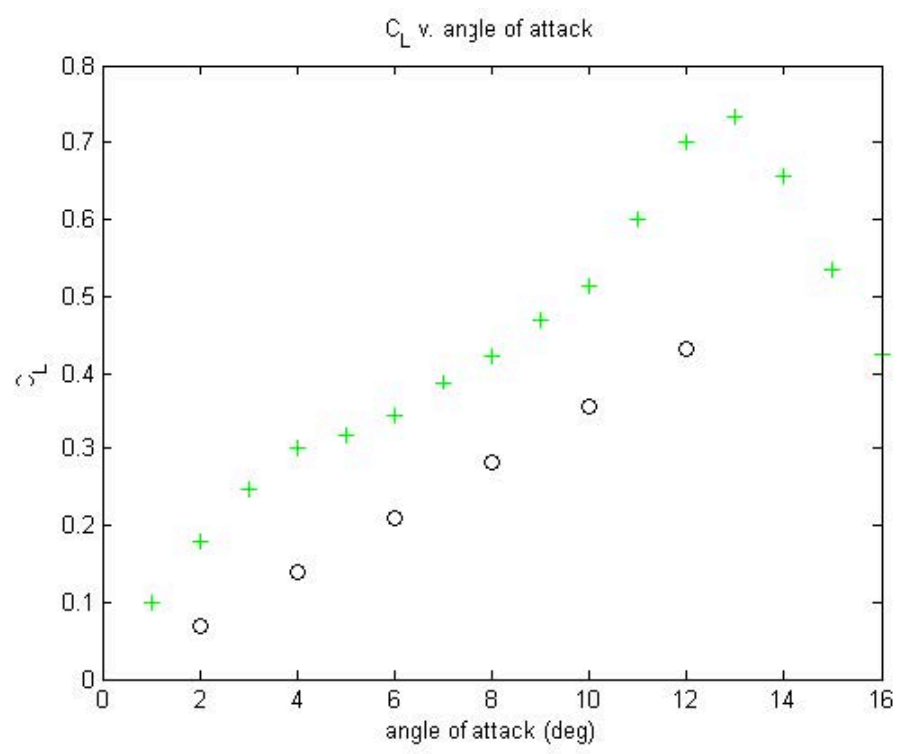

Figure J - PISO pressure-velocity coupling scheme: 16192 cells.

The PISO scheme lift has a slightly more shallow slope than the SIMPLE scheme and the values are still too low by at least 0.1 , which is still significant. Note, this simulation is the only one to use a pressure-velocity coupling scheme other than SIMPLE. The PISO scheme was used only to examine the effect of pressure-velocity coupling effect on solution. The results are similar enough that further cross-examination of PISO did not seem necessary. In general, the predictive ability of these models is considered limited. 
While they do capture the slope of the lift curve, they do not well match the actual experimental values for lift coefficient.

While the square shape results are not as satisfying as anticipated, the trailing edge (and subsequent aerodynamics/hydrodynamics) is very different from a D-shape. This difference in bluff body geometry, combined with the larger range of angle of attack, suggests that the D-shape should be a second, independent measure of CFD as an evaluation tool. Figure K below shows the simulated lab frame lift for the D-shape.

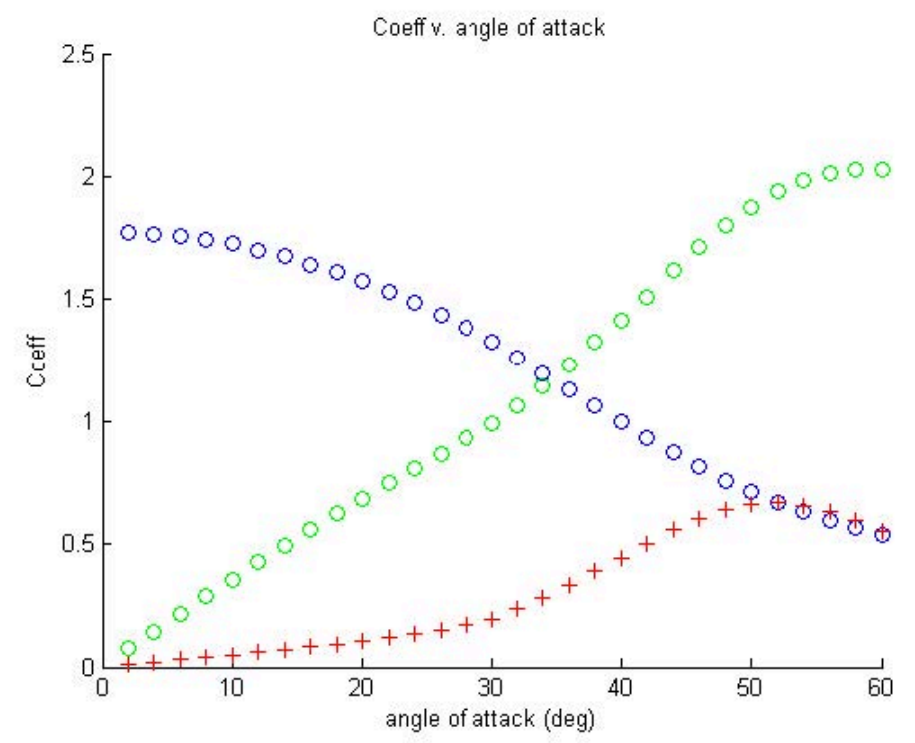

Figure K - D-shaped prism simulation: (green) lab-frame lift, (blue) lab-frame drag, (red) body-frame lift: 8033 cells.

After a coordinate transformation to account for the relative motion of the body to the fluid, the body frame lift is then calculated. The experimental values are then compared with the simulated values in Figure L below. 


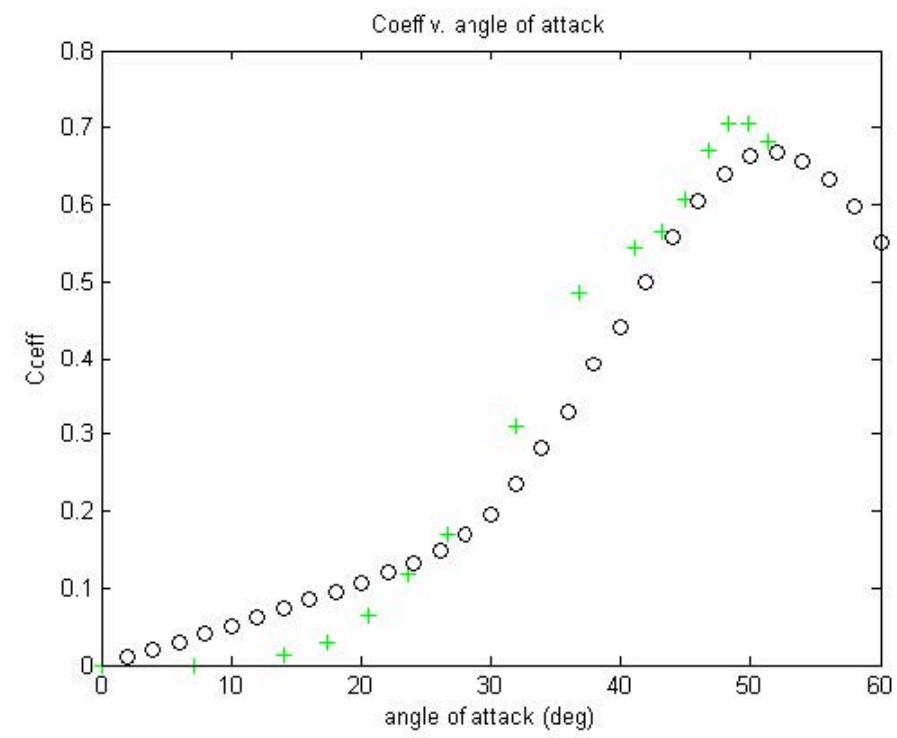

Figure L - D-shaped prism body-frame lift. (black) simulated, (green) experimental, source \{Novak1972\}: 8033 cells.

The simulation matches well with experimental data. These simulations are done with a $\mathrm{y}^{+}$value of approximately 30 for the nearest-wall cell center, as suggested by the ANSYS theory guide. One should notice that the D-shape simulated result appears to be a much better match than the square simulated result. The question is, why is this mesh apparently suitable for the D-shape but very poor for the square? The geometries are different, as noted above. The square's sharp trailing corners are a feature that is very much lacking in the D-shape. The subsequent flow separation that occurs for a square shape due to the sharp corners induces a large adverse pressure gradient that is likely not present for the D-shape. The flow domain, as a whole, is likely to be less turbulent for the D-shape than for the square, especially as angle of attack increases.

The equilateral triangle and trapezoid have also been considered as galloping oscillators. These are modeled with similar mesh detail to that of the D-shape, but corroborating experimental data is not available. Care must be taken with the evaluation of results. Again, a $y^{+}$value of approximately 30 is used for the nearest-wall cell center, as the trailing edges are sharp, but not right angles. Figure $\mathrm{M}$ below depicts the simulated results for the equilateral triangle. 


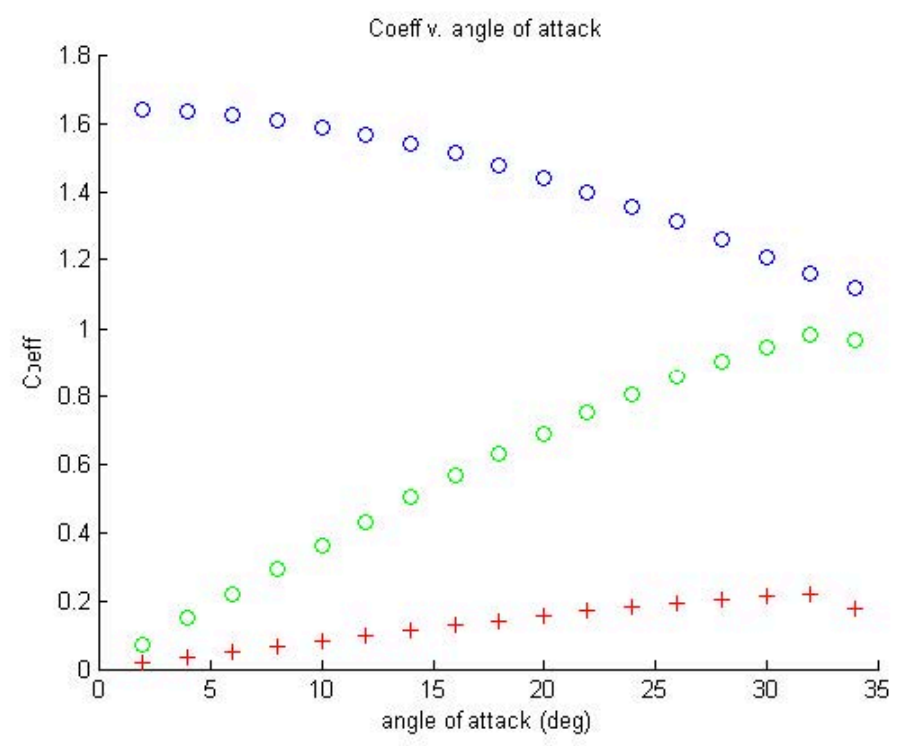

Figure M - Equilateral triangle shaped prism simulation. (green) lab-frame lift, (blue) lab-frame drag, (red) body-frame lift: 7344 cells.

The lab frame lift begins to decline near $34^{\circ}$, which translates to a corresponding drop in the body frame lift. The triangle is similar to a square in that it has sharp corners.

Figure $\mathrm{N}$ below shows the simulated result for the trapezoid.

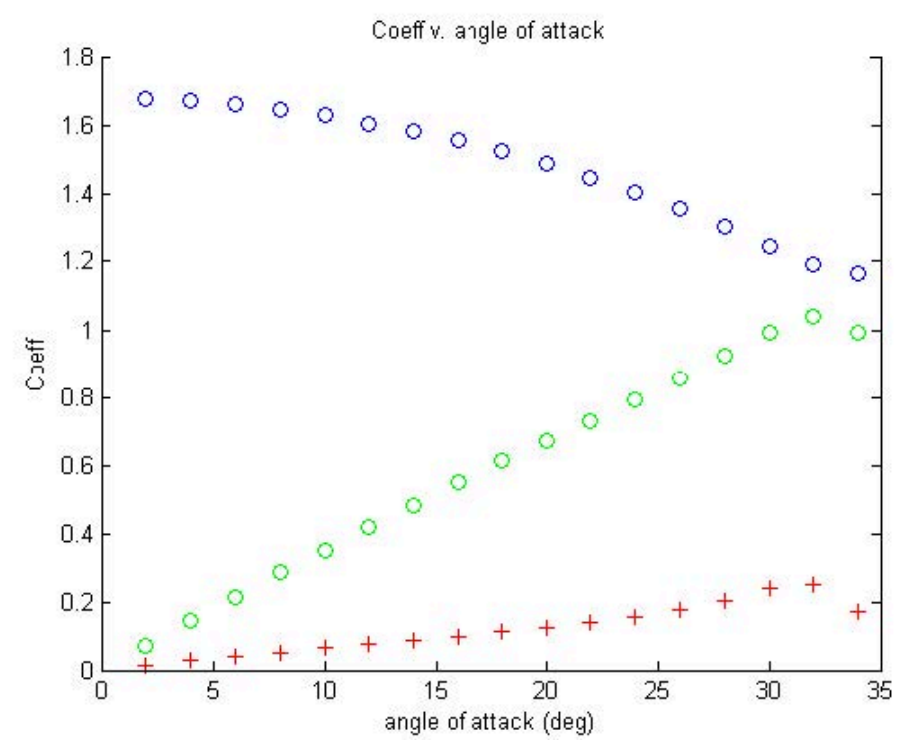

Figure N - Trapezoidal shaped prism simulation. (green) lab-frame lift, (blue) lab-frame drag, (red) bodyframe lift: 8033 cells.

Both the triangle and trapezoid shapes have similar simulated body frame lift coefficients. Much the same as with the equilateral triangle, the trapezoid also has sharp corners. It should be kept in mind that the shape of these curves is useful for exploring 
the characteristics of the describing function solution for coefficient of performance even if the data are not verified.

\section{GHEED Generations}

The GHEED Generations portion of the program embodied two primary goals:

(1) Initiate the formation of a database of the relative performance of galloping prisms of varied geometry, size, and/or weight; and

(2) Create an alpha-phase experimental prototype of a GHEED in order to preliminarily assess the energy available for harvest via the physical mechanism of gallop.

Regarding the first of these goals, the need for database development derives first from the reality that gallop has traditionally been viewed as undesirable, and thus literature review offers little insight into gallop-enhancing geometries. But in addition, that data which is available in literature is almost universally performed in laminar flows whereas the GHEED technology is intended for 'ill-behaved' flow regimes. In the long term, development of a GHEED-appropriate database can guide morphing development goals. In the short term it enables identification of especially effective rigid geometries for use in alpha-phase prototype development. In all, 14 rigid prisms and 1 morphing prism were dynamically characterized.

Regarding the second of the primary goals, even in the absence of coupling gallop to a DEG and morphing, it is intuitive that exploration of the fundamental concept of gallop for hydropower energy harvest is warranted. But in addition, during this program, working toward development of a viable GHEED prototype enabled (i) direct comparison of morphing vs. rigid prisms, and (ii) inspection of the effects of design changes. In all, three generations ('Gen-1', 'Gen-2', and 'Gen-3') were constructed and tested. Key components present in all three generations were: a galloping prism, variable suspension, and bearings; Gen-3 also included an EMI generator. As expected, performance improved with each generation, but more importantly, the evolution enabled identification of which parameters will play the most significant role in achieving further, post-program improvements.

\section{Database Development:}

The first phase of database development took an Edisonian approach to assessing the gallop cut-in speed of several rigid prisms using the Gen-1 device (Figure P) in the University of Pittsburgh ("Pitt") flume which is illustrated in Figure Q. Only prisms with geometries previously reported to display significant gallop, albeit in laminar flows, were selected. In addition, more than one orientation was tested for most prisms; the motivation for considering different orientations derives from the established fact that gallop of a D-shaped prism occurs when flow impinges on the flat side of the prism, but does not occur when flow is directed toward the curved surface. 

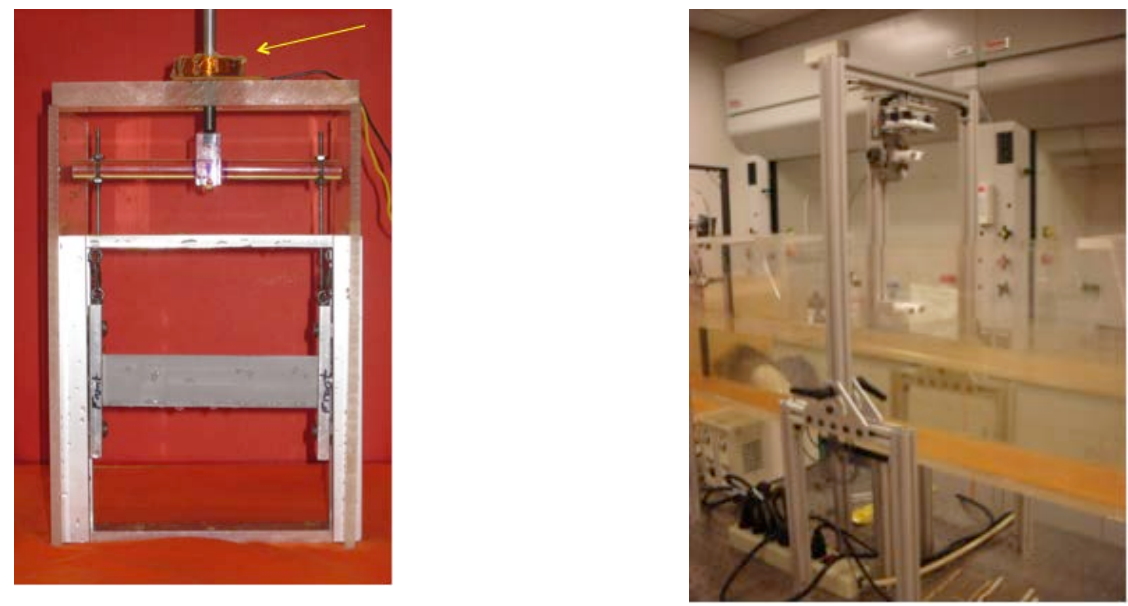

Figure P - (left). The First Generation GHEED ("Gen-1").

Figure Q - (right). The University of Pittsburgh (“Pitt”) flume.

Those prisms which displayed gallop were then subject to follow-on testing where an electromagnetic induction sensor was introduced to the Gen-1 device (indicated by the yellow arrow in Figure P) in order to estimate relative prism performance.

The final step in database development adapted the most promising prisms tested to that point, including one morphing prism, for dynamic characterization in the Gen-2 GHEED.

\section{GHEED Design Evolution:}

In the early stages of the program it was anticipated that the fundamental frame structure of the Gen-1 device would be employed throughout the program, with increasing complexity being either added directly to the Gen- 1 frame, or rebuilt but per the same basic vision. The rationale was that the Gen-1 device galloped well in the Pitt laboratory flume. However, as a matter of curiosity, the Gen-1 device was taken to a local stream early in the program, where it unexpectedly performed poorly. It was concluded that, while the frame structure was convenient from a design perspective, that in an open flow it created undesirable end effects. Thus, Gen-2 (Figure R) was constructed with a pedestal design that did not extend the full width of the flume. 


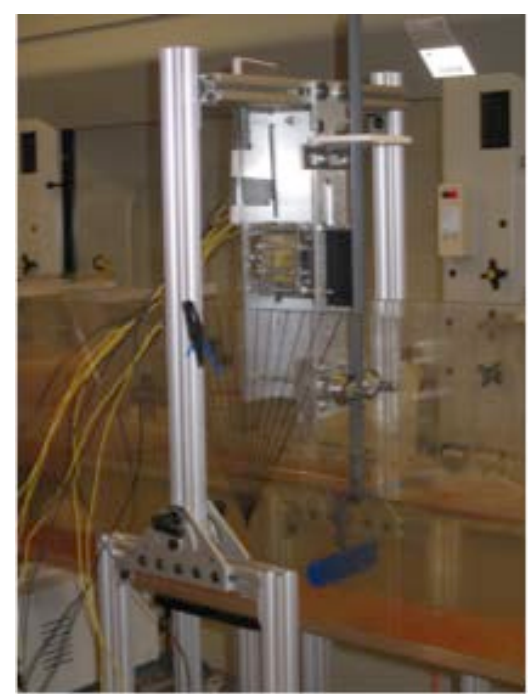

Figure R - The Second Generation GHEED (Gen-2) transitioned to a pedestal design to better emulate inriver loading conditions.

Finally, it was concluded that development of an experimental understanding of interfacing gallop with a generator would dramatically enhance the rate of technology development; thus creation of a power generating Gen-3 was added to the program goals. Specifically, assessment of generator-induced damping was desirable, which could be most effectively accomplished via introducing a generator with variable air-gap control (Figure S). The generator plates were designed and constructed at Pitt while the coils were produced by AMI; the partially assembled system is illustrated in Figure T. The resulting Gen-3 device, which retained the pedestal design of Gen-2, is illustrated in Figure V. 


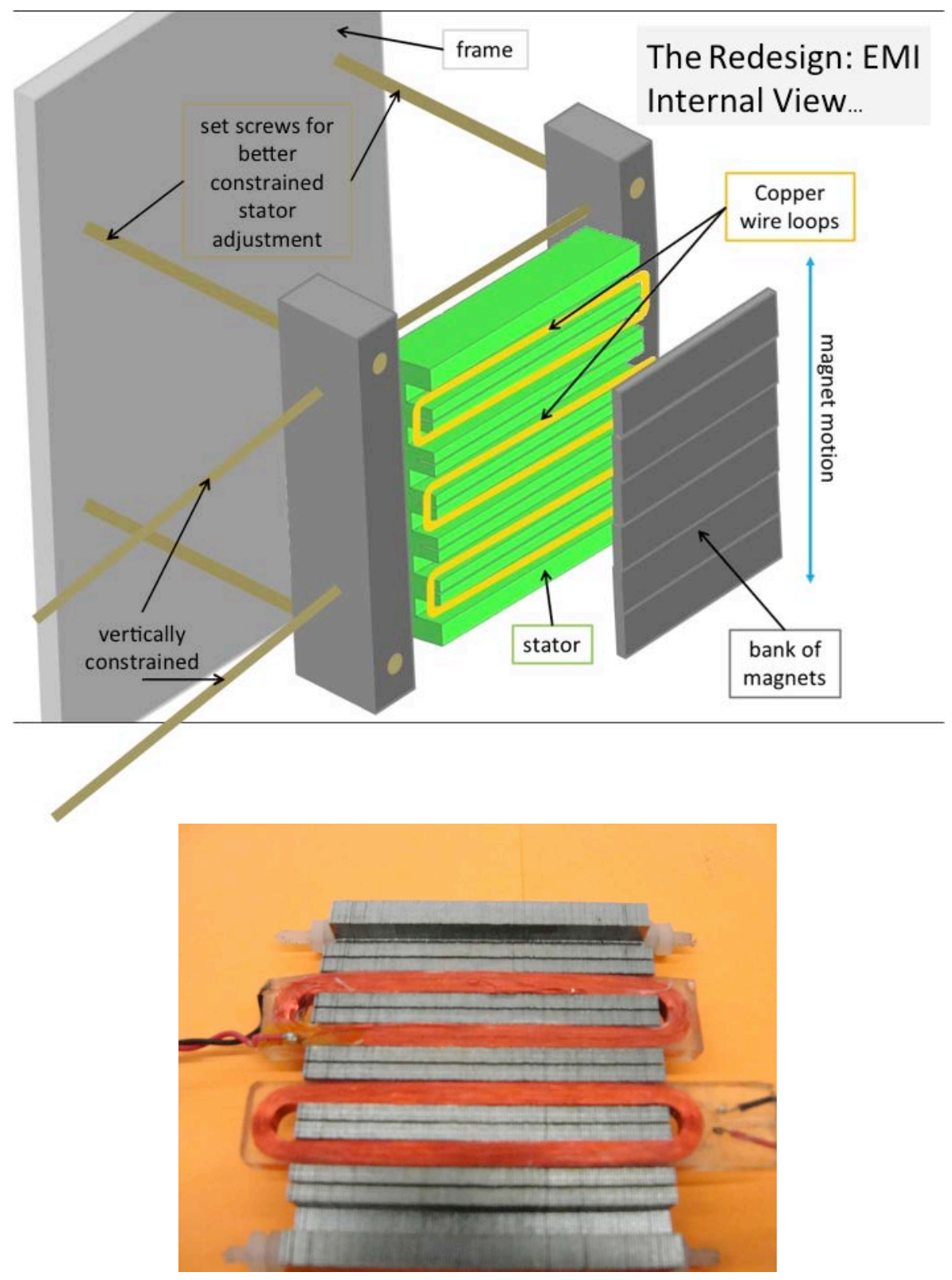

Figure S (above). Design of Variable Air Gap Control which was introduced in the Third Generation GHEED (“Gen-3”)

Figure $\mathbf{T}$ (below). The resulting stator, created collaboratively by Pitt and AMI

Because Gen-3 was intended to showcase the cumulative developed understanding, a rigorous testing protocol was defined for a 1" diameter D-prism with combined weight (prism, connector rod, and generator magnets) of $373 \mathrm{~g}$. Testing assessed:

1. Three different air gap distances within the EMI generator $(7,4$, and $3 \mathrm{~mm})$ in order to assess the trade-off between enhanced harvest expected with a reduced air gap versus the corresponding increase in mechanical damping to the galloping oscillator; 
2. Tests for each air gap were performed for at least 3 flow rates (either 0.55, 0.50, and $0.45 \mathrm{~m} / \mathrm{s}$ or $0.55,0.45$, and $0.34 \mathrm{~m} / \mathrm{s}$ depending on the observed cut-in flow speed);

3. Each combination above was tested for 3 system suspension cases $(107,142$, and $165 \mathrm{~N} / \mathrm{m})$;

4. Each combination above was tested for at least 4 electrical load resistances $(1,50$, 100, and 1000 ohms); and

5. Each combination above was tested 5 times so that an error bar assessment could be made. Each test included assessment of gallop dynamics via an accelerometer as well as power harvested.

In addition to the battery of tests described, and in light of the fact that gallop is an inherently unstable phenomenon, a repeatability test was performed on the device where it was tested, disassembled, reassembled, and then re-tested.

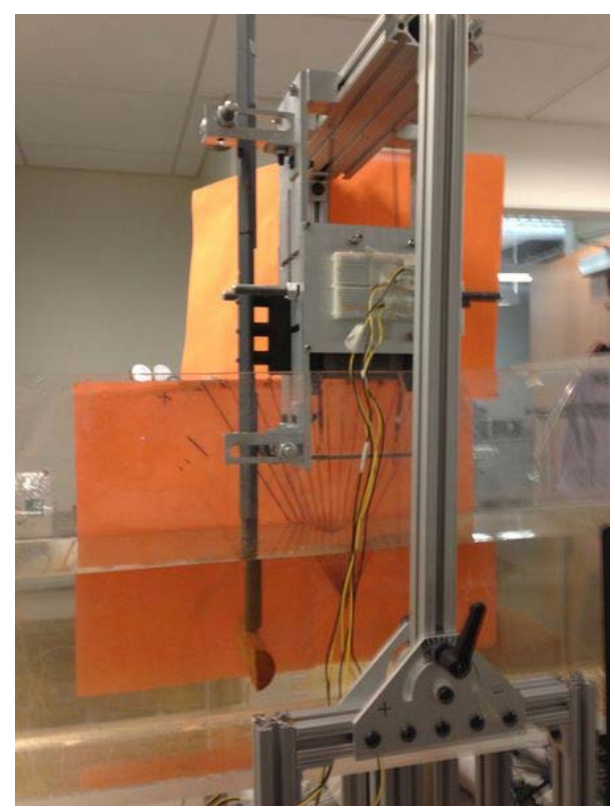

Figure V - The Third Generation GHEED (“Gen-3”) introduced a variable air gap electromagnetic generator in order to assess damping implications of energy conversion.

\section{Morphing}

Gallop is a mechanically vigorous behavior, making it intriguing for the purposes of energy harvest. However, it is an inherently unstable phenomenon. The purpose of the morphing component of this program was to establish that (i) morphing could be used to exert control over gallop response, and (ii) that a morphing design strategy could be developed. This effort took place in the following phases: 
1. Based in part on preliminary results from database development, a morphing target shape was selected;

2. A corresponding static design methodology was developed;

3. The design method was employed to fabricate a few specific morphing prisms. These prisms were subject to static loading, where response was compared to that projected per the design methodology; and

4. A preferred morphing prism was selected for dynamic testing.

The first of these steps, of necessity, was taken very early in the program. Both a Dshaped prism and a C-shaped prism were considered for morphing. The preliminary tests of each suggested that the morphing C-shaped prism would be preferred. One reason the C-shape was preferred was that it would likely be more effective at demonstrating a morphing design methodology. But equally important, this decision also derived from observations of significant trailing edge effects from the parallel prism database effort, where the C-shape had the ability to demonstrate an evolving trailing edge.

A design methodology was then established, aiming to control the extent to which the Cshape would be disposed to flaring open as a direct consequence of the flow rate induced dynamic pressure. The method was derived from Castigliano's Theorem for curved beams. In particular, the method addressed the application of anisotropy and/or varied beam thickness to affect the desired passive response to evolving flow boundary conditions.

The design equations were subsequently used to guide design of two morphing C-shaped prisms. These prisms were rapid prototyped with different materials. These prisms were subject to static tests inside the flume, where fluid flow rate was imposed to passively vary the C-shape. A video extensometer was used to track the opening of the tips via contrast marks on the prism tips. In addition, coupons of the rapid prototype materials were tested in a load frame so that appropriate material properties could be employed in the design equations. Finally, the experimental responses of the morphing prisms were compared to those predicted by the design equations.

The final step of the morphing methodology was to assess the better of the morphing prisms in dynamic (gallop) testing, per the same methods employed for the database development. To accomplish this, companion rigid prisms were also rapid prototyped and tested for comparison: a rigid prism conforming to the un-morphed (or un-flared) configuration and a rigid prism conforming to the fully-morphed (or fully-flared) configuration.

\section{Dielectric Elastomer Generator testing}

Modeling the equations and theory behind the numerical simulation of acrylic film Dielectric Elastomer Generator's (DEG) is based on data produced by testing of such devices at the Stanford Research Institute (SRI). The report starts with a short 
review of the data, analysis programs and DEG operation. Then the equations for a single DEG operating under energy harvesting conditions are developed followed by the equations for two coupled DEG's. The simulation for the coupled DEG's has the benefit of removing certain nonlinearities in the modeling, thereby reducing model complexity. A discussion of the mobility analogy, which is used to generate characteristics describing DEG operation and power scaling relations, follows.

The test data upon which the simulations are based come in two forms; the two element data and the four element data. An element is a thin film of electroactive polymer, in this case acrylic, that is stretched between two circular supporting frames. The frames at SRI allow for a total of five concentric elements to be connected parallel to each other. Elements are number from the outer element to the inner element as shown below.

\section{Element number}

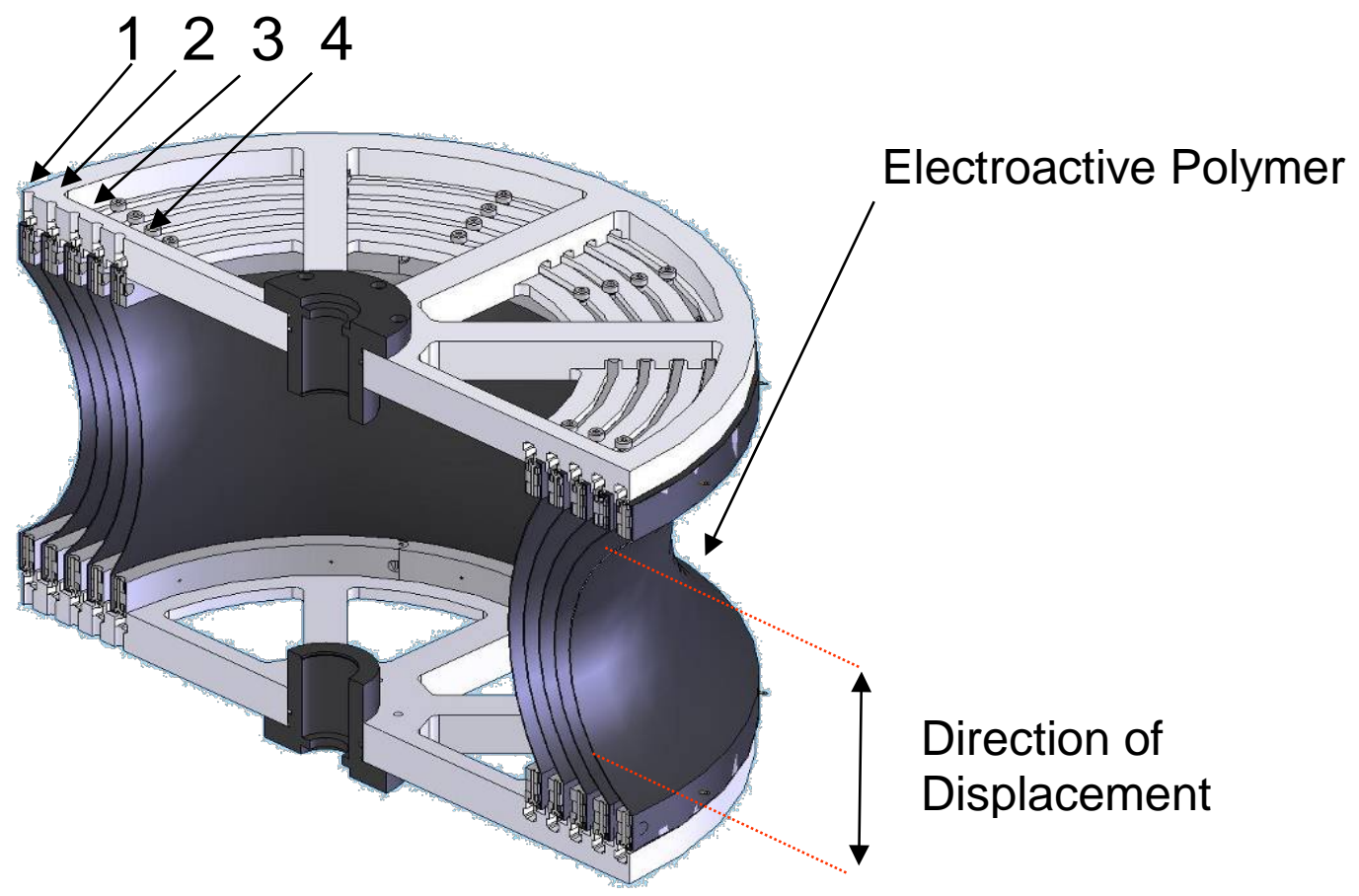

Figure W - SRI Acrylic DEG test unit.

In addition to testing with different element numbers, the tests were also done at different oscillation frequencies and seed voltages. The seed voltages that were used were $500 \mathrm{~V}, 1000 \mathrm{~V}$, and $1500 \mathrm{~V}$, while the frequencies that were used were $0.175 \mathrm{~Hz}, 0.352$ $\mathrm{Hz}, 0.529 \mathrm{~Hz}, 0.707 \mathrm{~Hz}, 0.885 \mathrm{~Hz}$, and $1.063 \mathrm{~Hz}$.

When an analysis of viscoelastic losses in the acrylic SRI generator indicated that another material would be more suitable for commercial development, additional material tests beyond the scope of the River Dream project were undertaken at Artificial Muscle (Figure X) 


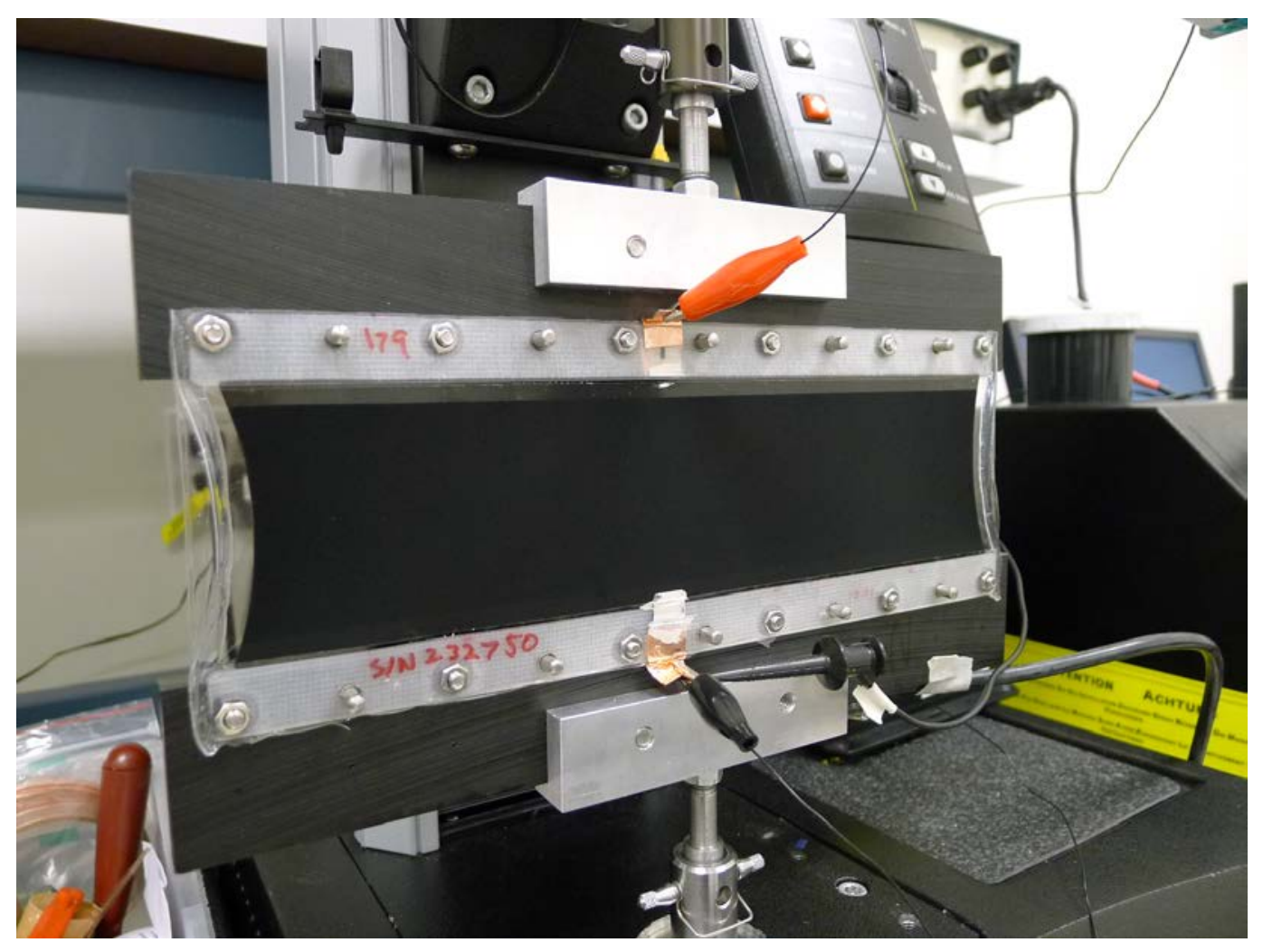

Figure X - A sample of dielectric elastomer generator material under test on the Instron at Artificial Muscle Inc.

\section{DEG modeling}

The goal of analyzing the data was to generate values and equations to be used in a DEG simulation that accurately models DEG operation and allows for optimization of a theoretical design. The analysis produced third order polynomial equations that were used to simulate the force and the capacitance. In addition, the analysis provided values for the mechanical and electrical energy losses in the system. The mechanical losses were found to be accurately simulated with viscous damping where the damping term is a function of the RMS velocity. Electrical losses can be accurately simulated by a resistor in parallel with the film.

The simulations were written in Matlab and PSPICE and the results of these different simulation platforms were compared to check for numerical and programmatic errors. The modeling of DEG's at AMI assumes that the system is driven by a sinusoidal velocity source. This requires the user to define parameters for this velocity source as well as resistor value for modeling the electrical losses. The necessary input parameters are given in the following table: 


\begin{tabular}{|c|c|}
\hline Parameter & Description \\
\hline$x_{\max }$ & Maximum displacement $[\mathrm{m}]$ \\
\hline$x_{\min }$ & Minimum displacement [m] \\
\hline$V_{\text {seed }}$ & Seed Voltage $[\mathrm{V}]$ \\
\hline$R_{E L}$ & Electrical energy loss equivalent resistance $[\Omega]$ \\
\hline$f$ & Frequency $[\mathrm{Hz}]$ \\
\hline$N$ & Number of cycles \\
\hline
\end{tabular}

Table 1 - Input parameters.

It is assumed that all of the input parameters are positive real numbers and $x_{\max }>x_{\min }$. The SRI data sets upon which the simulations are based place further constraints on the range of the parameters:

$$
\begin{gathered}
0.8255 \leq x_{\min }<x_{\max } \leq 0.2 \\
500 \leq V_{\text {seed }} \leq 1500 \\
0.175 \leq f \leq 1.063
\end{gathered}
$$

The rest of the variables that are used in this report are given in the following tables:

\begin{tabular}{|c|c|}
\hline Variable & Description \\
\hline \hline$\Delta t$ & Time step [s] \\
\hline$t$ & Time [s] \\
\hline$u$ & Oscillation Amplitude [m] \\
\hline$x$ & Velocity $\left[\frac{m}{s}\right]$ \\
\hline$C$ & Displacement [m] \\
\hline$F_{S}$ & Capacitance [F] \\
\hline$C_{\text {max }}$ & Elastic Force [N] \\
\hline$C_{\min }$ & Maximum Capacitance [F] $]$ \\
\hline$Q_{s e e d}$ & Minimum Capacitance [F] $]$ \\
\hline$V$ & Seed Charge [Coulombs] \\
\hline$V_{\text {max }}$ & Voltage [V] $]$ \\
\hline$c_{M L}$ & Maximum Voltage [V] $]$ \\
\hline$\delta$ & Mechanical loss viscous damping coefficient $\left[\frac{N S}{m}\right]$ \\
\hline$\tau$ & Parameter in equation for $c_{M L}$ \\
\hline$F_{c}$ & Parameter in equation for $c_{M L}$ \\
\hline$F_{E}$ & Damping force [N] \\
\hline$F_{M}$ & Electrostatic force [N] \\
\hline$W_{E L}$ & Mechanical force [N] \\
\hline$W_{M L}$ & Average electrical energy loss per cycle $\left[\frac{J}{c y c l e}\right]$ \\
\hline$W_{E}$ & Average mechanical energy loss per cycle $\left[\frac{J}{c y c l e}\right]$ \\
\hline$W_{M}$ & Electric potential energy [J] \\
\hline$W_{H}$ & Instantaneous mechanical energy $[\mathrm{J}]$ \\
\hline & Energy harvested per cycle $\left[\frac{J}{c y c l e}\right]$ \\
\hline
\end{tabular}

Table 2 - Single DEG variables. 


\begin{tabular}{|c|c|}
\hline Variable & Description \\
\hline \hline$u_{1}$ & DEG 1 velocity $\left[\frac{m}{s}\right]$ \\
\hline$u_{2}$ & DEG 2 velocity $\left[\frac{m}{s}\right]$ \\
\hline$F_{S 1}$ & DEG 1 elastic force [N] \\
\hline$F_{S 2}$ & DEG 2 elastic force [N] \\
\hline$F_{S 1+S 2}$ & Net elastic force [N] \\
\hline$F_{c 1}$ & DEG 1 damping force [N] \\
\hline$F_{c 2}$ & DEG 2 damping force [N] \\
\hline$F_{E 1}$ & DEG 1 electrostatic force [N] \\
\hline$F_{E 2}$ & DEG 2 electrostatic force [N] \\
\hline$R_{H}$ & Energy harvested equivalent resistance $[\Omega]$ \\
\hline
\end{tabular}

Table 3 - Coupled DEG variables.

\begin{tabular}{|c|c|}
\hline Variable & Description \\
\hline \hline$R$ & Resistance $[\Omega]$ \\
\hline$r$ & Mechanical responsiveness $\left[\frac{\mathrm{m}}{\mathrm{NS}}\right]$ \\
\hline$c$ & Viscous damping term $\left[\frac{N S}{\mathrm{~m}}\right]$ \\
\hline$I$ & Current [Amperes] \\
\hline$r_{E L}$ & Electrical energy loss equivalent mechanical responsiveness $\left[\frac{\mathrm{m}}{N s}\right]$ \\
\hline$r_{H}$ & Energy harvested equivalent mechanical responsiveness $\left[\frac{\mathrm{m}}{\mathrm{NS}}\right]$ \\
\hline$c_{E L}$ & Electrical energy loss equivalent viscous damping term $\left[\frac{N s}{\mathrm{~m}}\right]$ \\
\hline$c_{H}$ & Energy harvested equivalent viscous damping term $\left[\frac{N S}{\mathrm{~m}}\right]$ \\
\hline
\end{tabular}

Table 4 - Mobility Analogy variables.

The free body diagram for a single DEG is shown below: 


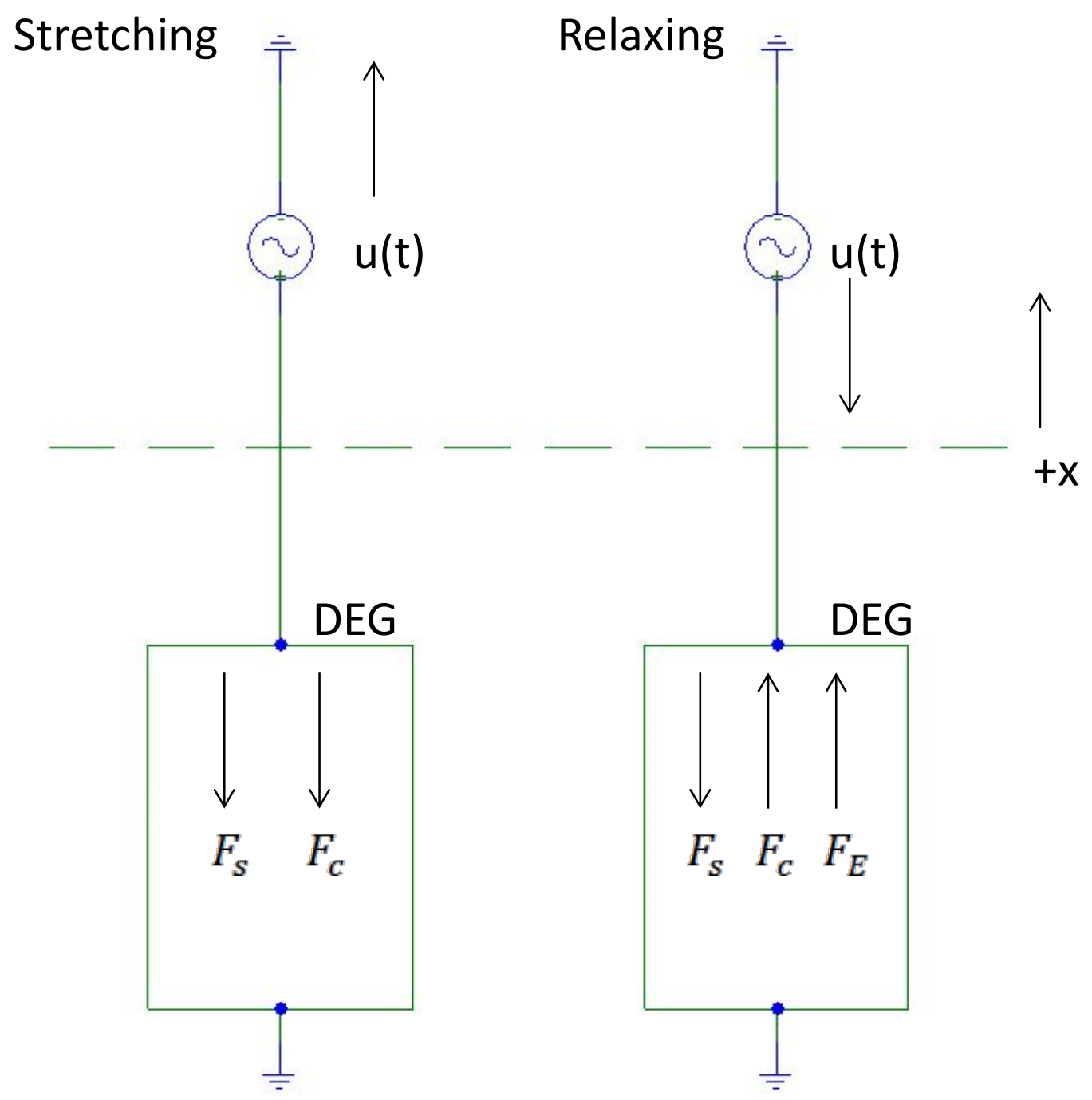

Figure $\mathbf{Y}$ - Single DEG free body diagram.

A typical DEG cycle is described in four steps, two of which were shown in the free body diagram. The four steps are listed below and labeled in the following voltage graph:

1. Stretching - DEG is stretched from its minimum to its maximum displacement.

2. At the maximum displacement, the seed voltage is applied to the film.

3. Relaxing - DEG is relaxed from its maximum displacement to its minimum displacement, which reduces the capacitance and increases the voltage.

4. When the DEG has reached its minimum displacement, the voltage is removed from the film and the energy is harvested. 


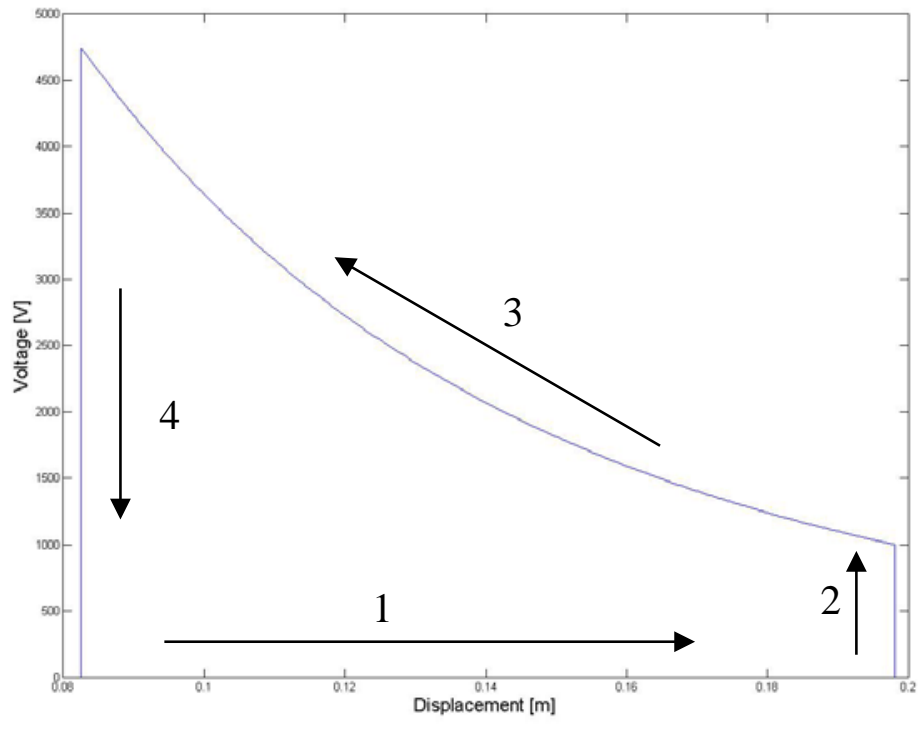

Figure Z - Voltage loop for a single DEG.

Note that when the displacement is at a maximum, the capacitance is also at a maximum, while the voltage is set to the seed voltage. When the displacement is at a minimum, the capacitance is also at a minimum while the voltage is at a maximum. This is summarized in the table below:

\begin{tabular}{|c||c|c|}
\hline Position & Capacitance & Voltage \\
\hline$x_{\max }$ & $C_{\max }$ & $V_{\text {seed }}$ \\
\hline$x_{\min }$ & $C_{\min }$ & $V_{\max }$ \\
\hline
\end{tabular}

Table 5 - Max-Min displacement conditions.

In the coupled DEG's scenario, two DEGs are connected to opposite sides of an oscillating prism or mass. Thus the two DEGs are operating 180 degrees out of phase with each other as shown in the free body diagram below, 


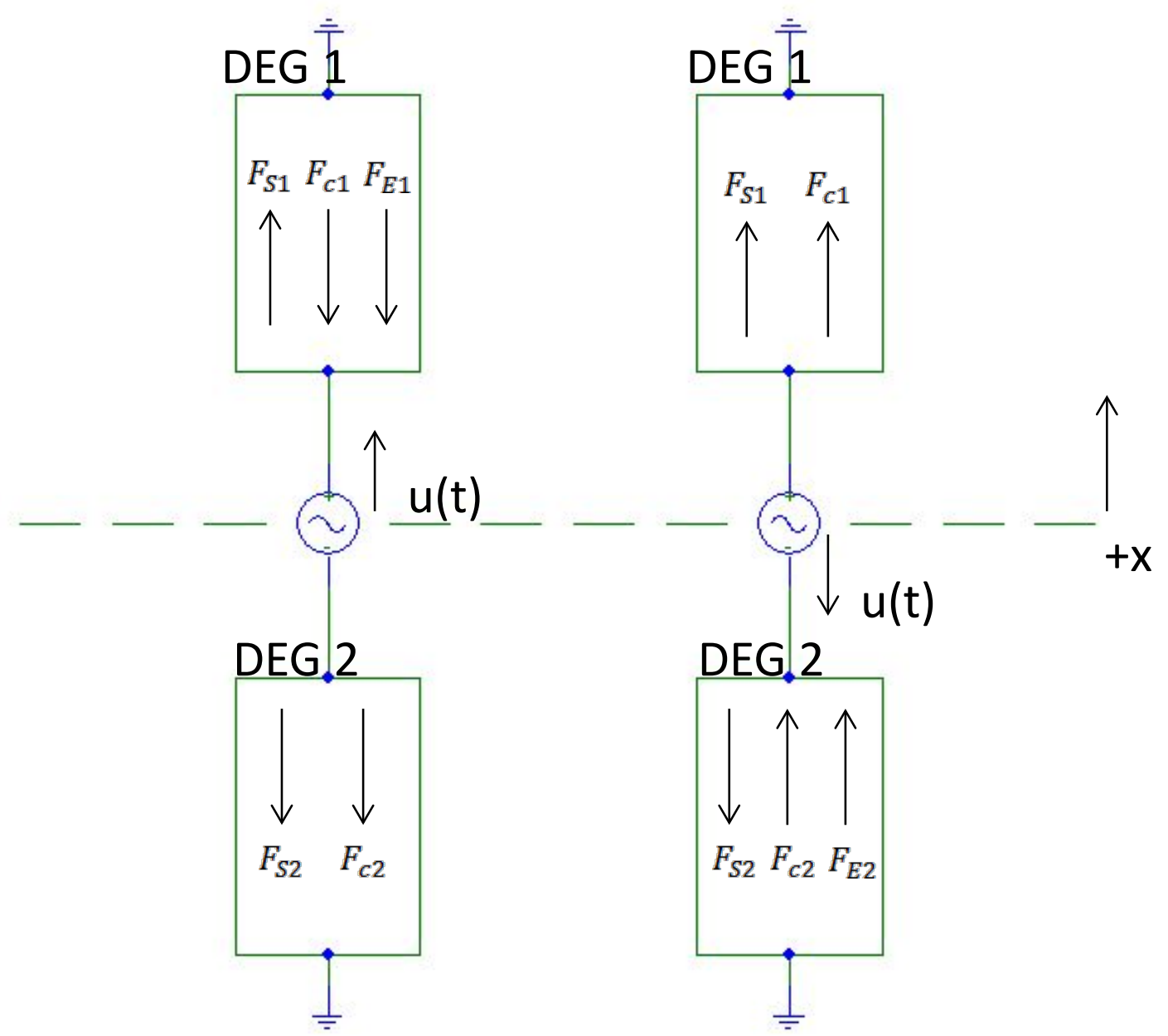

Figure AA - Coupled DEG free body diagram.

Although it may seem that this scenario is more complicated than the single DEG simulation, it turns out that the nonlinearities exhibited in the elastic force of a single DEG are removed in the coupled configuration

\section{RESULTS AND DISCUSSION}

This section presents and discusses the results from implementing the above described methods. Identical sub-section headings are employed to facilitate review where applicable.

\section{Galloping Oscillator}

We investigated the characteristics of a galloping device under laboratory conditions in the University of Pittsburgh's flume. The objective of this effort was to determine the characteristics of the limit-cycle oscillations and estimate the magnitude of the energy that can be harvested with such a device. 
This section further discusses the results of experiments made with the Gen-3 design that are also discussed in EXPERIMENTAL - GHEED Design Evolution Section previously. Each test included assessment of gallop dynamics via an accelerometer as well as power harvested. The testing conditions that were assessed are:

1. Three different air gap distances within the EMI generator $(7,4$, and $3 \mathrm{~mm})$ in order to assess the trade-off between enhanced harvest expected with a reduced air gap versus the corresponding increase in mechanical damping to the galloping oscillator;

2. Tests for each air gap were performed for at least 3 flow rates (either $0.55,0.50$, and $0.45 \mathrm{~m} / \mathrm{s}$ or $0.55,0.45$, and $0.34 \mathrm{~m} / \mathrm{s}$ depending on the observed cut-in flow speed);

3. Each combination above was tested for at least 4 electrical load resistances $(1,50$, 100 , and 1000 ohms); and

4. Each combination above was tested 5 times so that an error bar assessment could be made. Each test included assessment of gallop dynamics via an accelerometer as well as power harvested.

In addition to the battery of tests described a repeatability test was performed on the device where it was tested, disassembled, reassembled, and then re-tested. Results are reproducible.

The Gen-3 design uses a linear electro-magnetic generator. As such, the effective damping is inversely related to the load resistance: $c=\kappa^{2} / R$ where $\kappa$ is the force/backemf constant. As such, for the results that follow, amplitude and power are plotted versus the conductance, $1 / R$, of the load.

Figure BB below shows the amplitude of the galloping response for various air-gap spacing distances and different flow rates, as outlined above. 


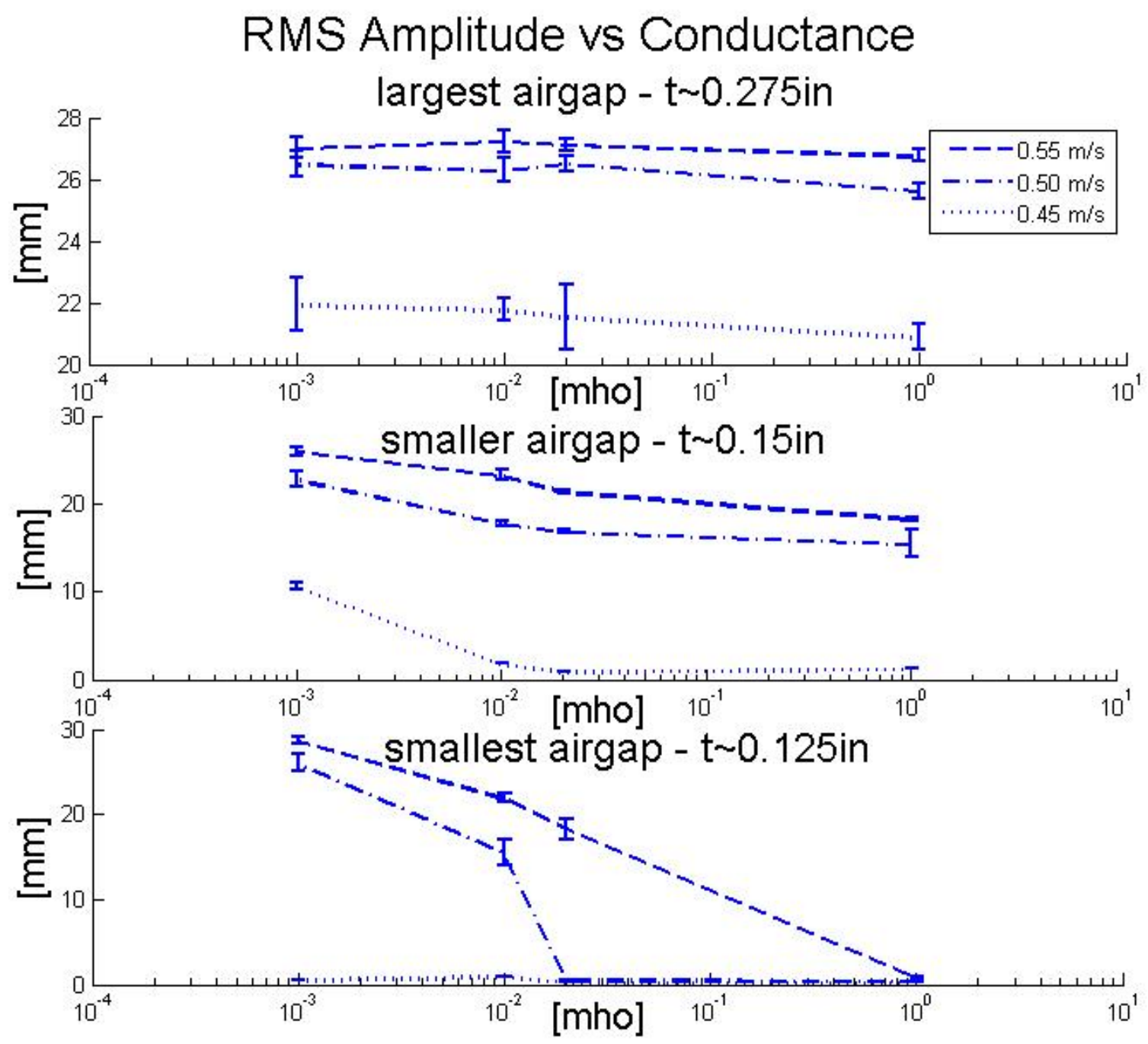

Figure BB - The RMS galloping amplitude for various air-gap spacing on the generator.

. Decreasing the air-gap spacing is related to an increase in the force constant, $\kappa$, although that coefficient was not measured. For the largest air-gap spacing, $7 \mathrm{~mm}(0.275$ in), at any particular flow speed, the amplitude is fairly insensitive to load; this is expected from theory presented previously. The amplitude somewhat does depend upon the flow speed, which is not expected from theory. It should be pointed out that the figure may be misleading since the amplitude for highest flow rate is near $27 \mathrm{~mm}$ and that for the lowest flow rage is near $22 \mathrm{~mm}$, a change of $-18 \%$. While the device does oscillate, it does not do so sinusoidally with constant amplitude. Rather, turbulence in the flume and other factors will make its amplitude fluctuate. It is likely that there are other non-linear effects in the harvester, its flow-dynamics, and the flow characteristics of the flume. For example, the flume's cross-sectional area is confined such that there are surface wave phenomena that affect the flow over the device.

More interesting is the fact that as the air gap decreases, implying an increasing force constant that the amplitude behavior of the device depends more heavily on flow speed and electrical load. At the smallest gap and lowest flow rate the amplitude is nearly zero. There are various non-linear effects in the characteristics of the electrical generator that 
affect the device's performance. At small air-gap spacing, the strong magnetic fields, which result in a high force constant, also result in localized dips in the magnetic potential. As a result, the magnetic force fluctuates with position, and the device can temporarily stall when the hydrodynamic force is not sufficient to overcome the associated magnetic force. The net result is that the device can both temporarily stall, necessitating a "kick" to displace it from its current position, or can oscillate with small amplitude.

Figure CC below shows the cycle average power generated by the device.

\section{AVG Power}
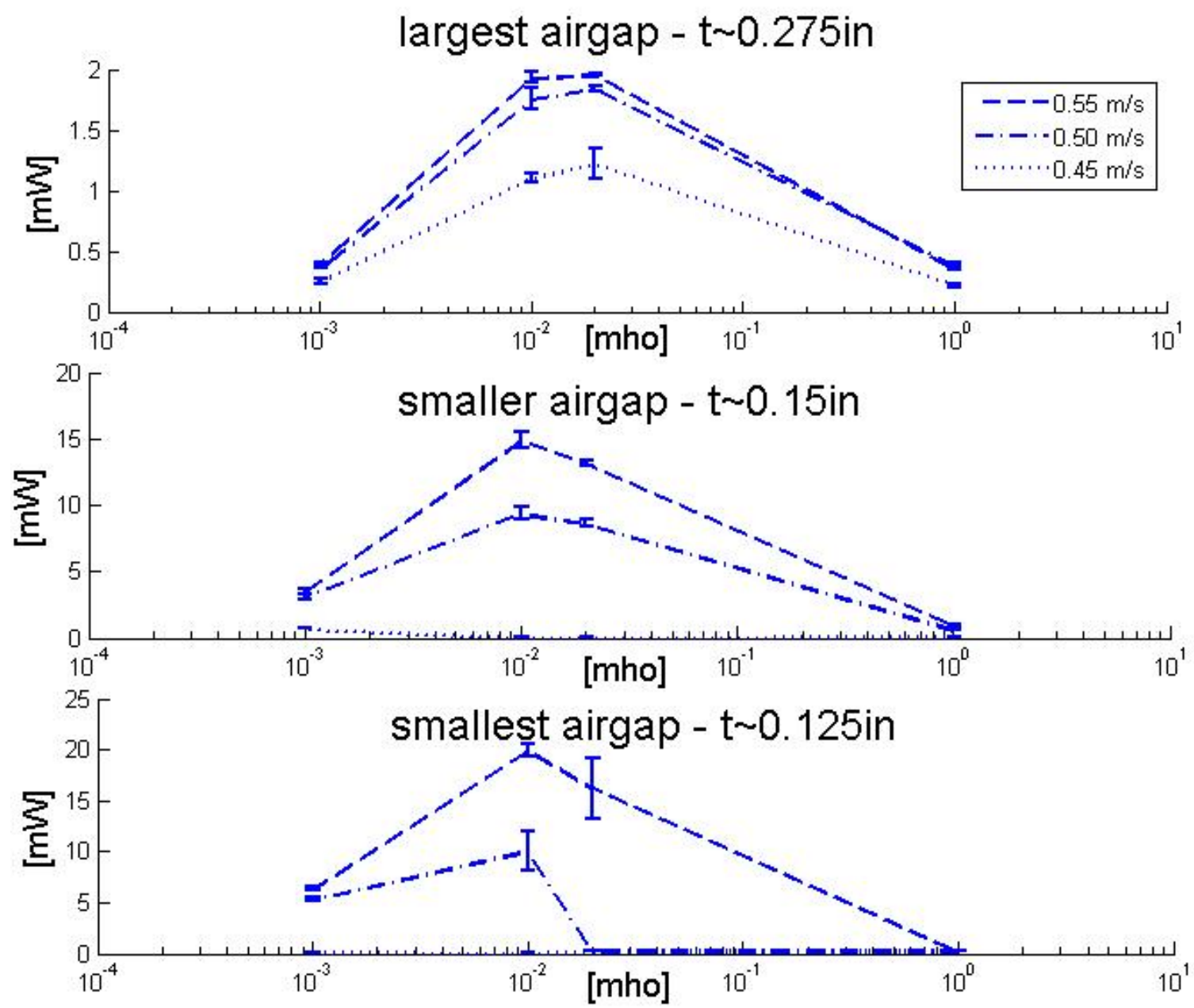

Figure CC - The cycle averaged power for various air-gap spacing on the generator.

Again, the device was tested for various air-gap spacing distances, flow speeds, and load resistances. In all cases, the power-load relationship is small at low conductance (low equivalent damper) rising to a peak value in the range of $10-20 \mathrm{mS}(50-100 \Omega)$ then decreasing for larger conductance (large equivalent damper). This behavior is expected. At low equivalent damping (low conductance) there will be no energy harvested since the harvester does not effectively convert mechanical energy to electrical energy to be used by the load. At high equivalent damping (high conductance) there is no energy harvested 
because either device cannot move (not the situation here) or there is not sufficient electrical current generated by the device to supply meaningful power the load. At some intermediate value of damper (conductance) the optimum amount of power is generated. Finding the optimum value of the load is an impedance matching problem. What is interesting for the results presented here is that this optimum impedance match appears to be largely independent of the characteristics of the electrical generator with all values falling largely in the same range. This conclusion is not certain since for the $3 \mathrm{~mm}$ and 4 $\mathrm{mm}$ air-gap spacing distances the true peak of the power curve may lie in the range of 1 to $10 \mathrm{mS}$. Theory suggests that the peak of the curve should occur at a value

$$
\beta_{o p t}=\frac{2 c}{\rho V S}=\frac{2 \kappa^{2}}{\rho V S R}
$$

Thus, as the air-gap gets smaller and the force constant, $\kappa$, gets larger, we should expect the optimum conductance, $1 / R$, to decrease. The data shown suggests this, although not conclusively. However, more power is harvested with decreasing air-gap with a gap of 3 mm generating nearly an order of magnitude (factor of 10) more power than a gap of 7 $\mathrm{mm}$. This is expected since the decreasing air-gap results in an increasing force constant and an increase in power generated.

$$
P_{\text {gen }}=\frac{1}{2} c \omega^{2} Y^{2}=\frac{\kappa^{2} \omega^{2} Y^{2}}{2 R}
$$

For the device, the frequency $\omega$ is depends primarily on the spring characteristics. The amplitude $Y$ does depend upon the flow and load characteristics, but can for this short discussion be assumed constant. Thus, the power generated increases as the force constant increases. From the factor of 10 increase in power generated, we might conclude that the force constant increased by about a factor of 3 , and that the peak of the power curve should shift to the left by a factor of 10 as well. While this is suggested by the data, nothing conclusive can be made.

For the data shown in Figure CC the most power is harvested at the highest flow speeds. This is expected since the coefficient of performance is

$$
C_{P}=\frac{P_{\text {gen }}}{q S V}=\frac{\frac{1}{2} c \omega^{2} Y^{2}}{q S V}=\frac{\kappa^{2} \omega^{2} Y^{2}}{\rho S V^{3} R} .
$$

Thus, the power generated should increase as the cube of the flow speed. We see this for the case of the largest air-gap, $7 \mathrm{~mm}(0.275 \mathrm{in})$. The power harvested is approximately 2 $\mathrm{mW}$ for a flow speed of $0.55 \mathrm{~m} / \mathrm{s}$. When the flow speed is reduced to $0.45 \mathrm{~m} / \mathrm{s}$, the power should decrease by a factor of 0.53 to a value of $1.09 \mathrm{~mW}$, which is what is seen. A similar behavior is seen for the gap of $4 \mathrm{~mm}(0.150 \mathrm{in})$ the peak power is approximately $15 \mathrm{~mW}$ for a flow speed of $0.55 \mathrm{~m} / \mathrm{s}$. When the speed is reduced to $0.50 \mathrm{~m} / \mathrm{s}$, the power decreases to approximately $11 \mathrm{~mW}$, a reduction by a factor of 0.75 as expected. Other non-linear effects come into play for the smallest air-gap spacing, so such conclusions cannot be made.

\section{Computational Fluid Dynamics}

The coefficient of performance (COP) was discussed in the Experimental section under Galloping Oscillator. There the COP as a function of normalized flow speed $\beta$ to be the roots of 


$$
\frac{35}{64} a_{7} C_{P}^{3}+\frac{5}{16} a_{5} \beta C_{P}^{7}+\frac{35}{64} a_{3} \beta^{2} C_{p}+\frac{1}{8} \beta^{3}\left(a_{1}-\beta\right)=0 .
$$

Figure DD below shows that the COP for various flow speeds and dimensions.

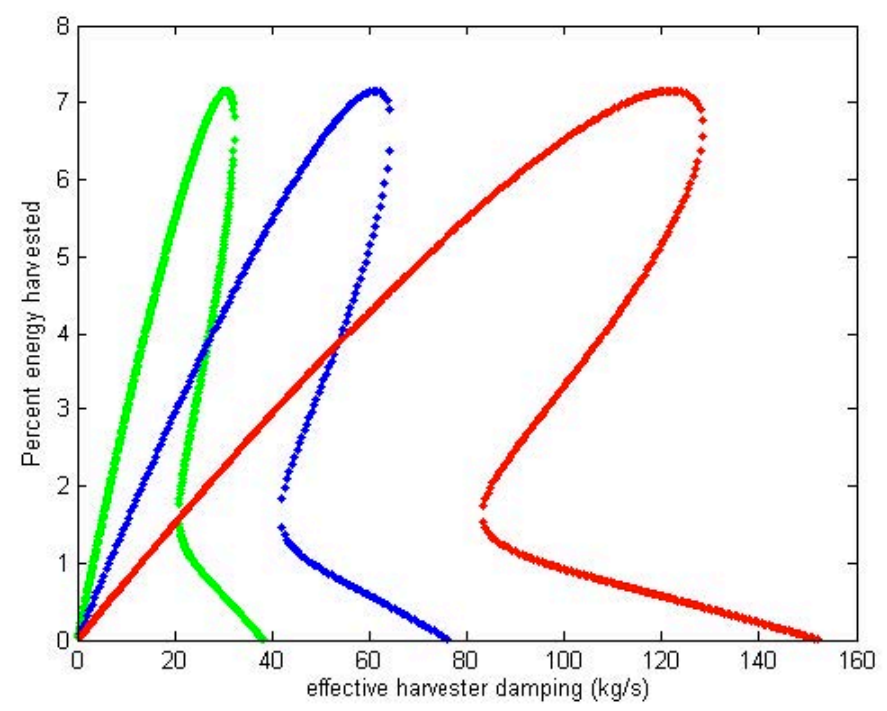

Figure DD - Coefficient of performance for a square prism using Parkinson \{Parkinson1964\} experimental data. Prism size 6” x 24”: (green) $1 \mathrm{ft} / \mathrm{s}$, (blue) $2 \mathrm{ft} / \mathrm{s}$, (red) $4 \mathrm{ft} / \mathrm{s}$.

While the COP does not change, the optimum value for effective harvester damping does vary with speed.

Similarly for the D-shaped prism, the predicted optimum COP is very similar between the experimental and CFD data. This suggests that the evaluation of the COP using CFDsimulated lift coefficient can be useful as long as the lift coefficient is accurate for a sufficiently large portion of the angle of attack range, including the peak lift. Similar to the square, the D-shape's optimum COP does not change with varied body size, but the amount of equivalent damping does vary. This result is shown in Figure EE. 


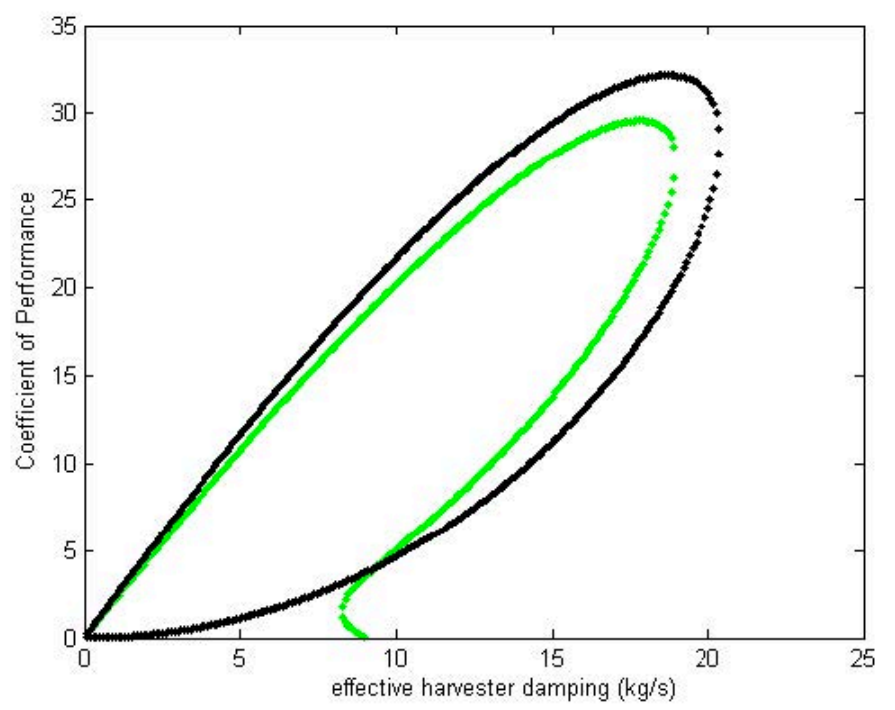

Figure EE - A comparison of the coefficient of performance for a D-shaped prism using experimental data and CFD simulations. Prism size 6" x 24", fluid velocity 2 ft/s: (green) simulated, (black) experimental.

The square-prism performance comparisons are different than the D-shape. For the square-prism, the peak and general performance predictions are poor. The simulated lift coefficient of performance is compared to the experimental lift coefficient \{Parkinson1964\} performance for the square in Figure FF.

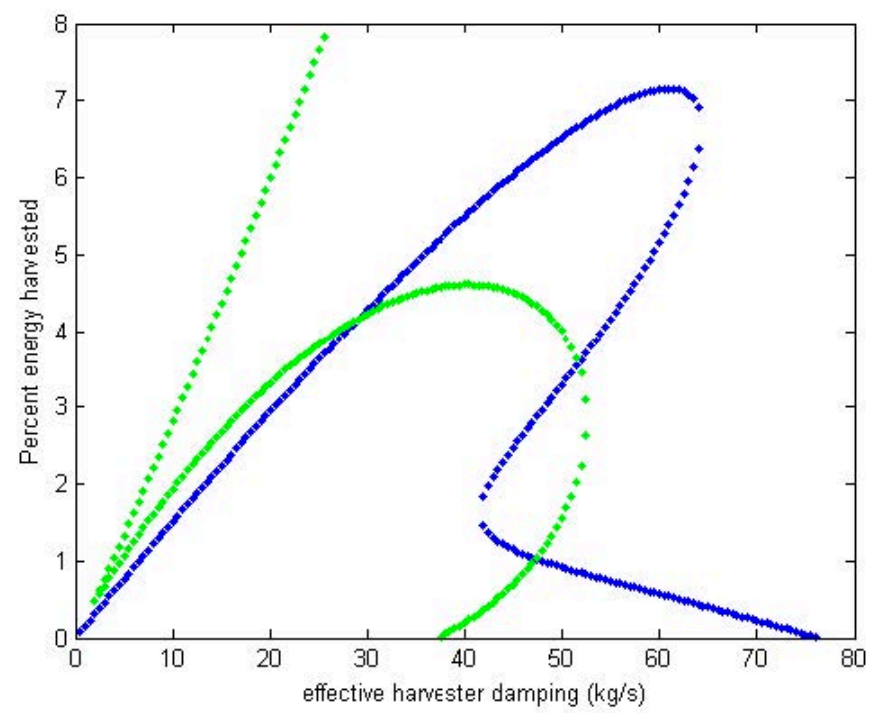

Figure FF - A comparison of the coefficient of performance for a square-prism between CFD simulated and experimental lift curves. Prism size 6” x 24”, fluid velocity $2 \mathrm{ft} / \mathrm{s}$. (green) simulated, (blue) experimental.

In these figures, the peak COP does not vary, but the effective damping permitted does vary by a factor directly proportional to the increase in prism characteristic area, which 
should be intuitively comfortable. One will also notice that the COP for the simulated data does not match well with the experimental COP for the square.

While the trapezoid and equilateral triangle lift curves do not have experimental data, COP curves can still be generated using CFD-simulated lift curves. The CFD simulated lift for both the square and D-shape appear to be conservative underestimates.

Figure GG shows the simulated lift coefficient versus angle of attack displayed for the trapezoid bluff body.

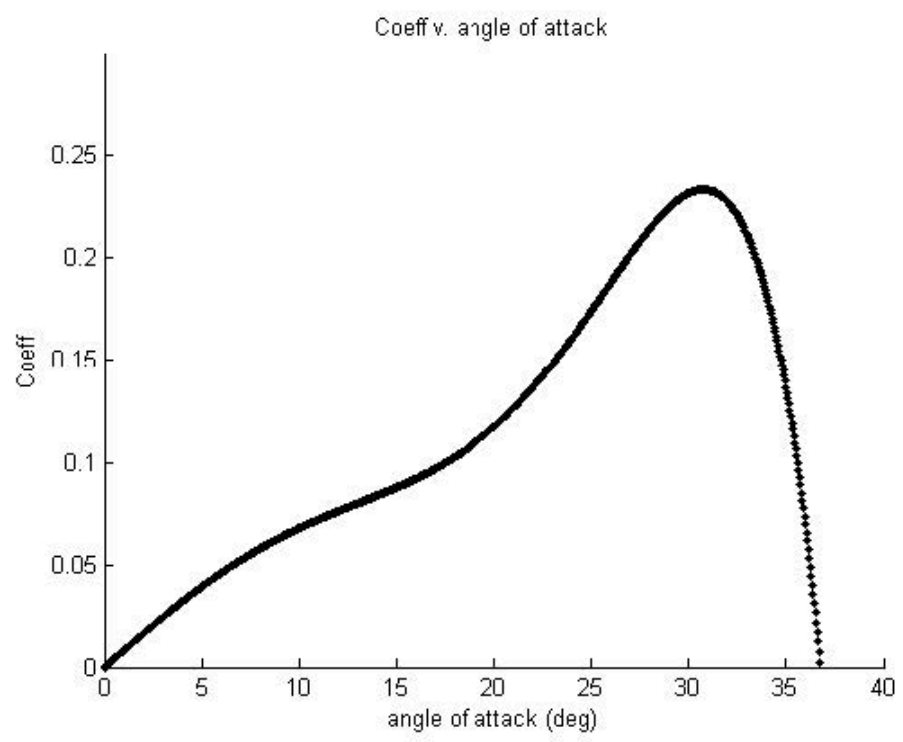

Figure GG - Coefficient of lift for a trapezoid. Prism size 1” x 6”, fluid velocity $2 \mathrm{ft} / \mathrm{s}$.

Interestingly, the shape is very similar to that of the experimental square lift curve.

However, when one notices the scale, the coefficient's magnitude is much smaller and the range for angle of attack is much greater, so those appearance qualities are only coincidence. Similarly, the COP curves in Figure HH are likewise similar in form to the experimental square performance curve. 


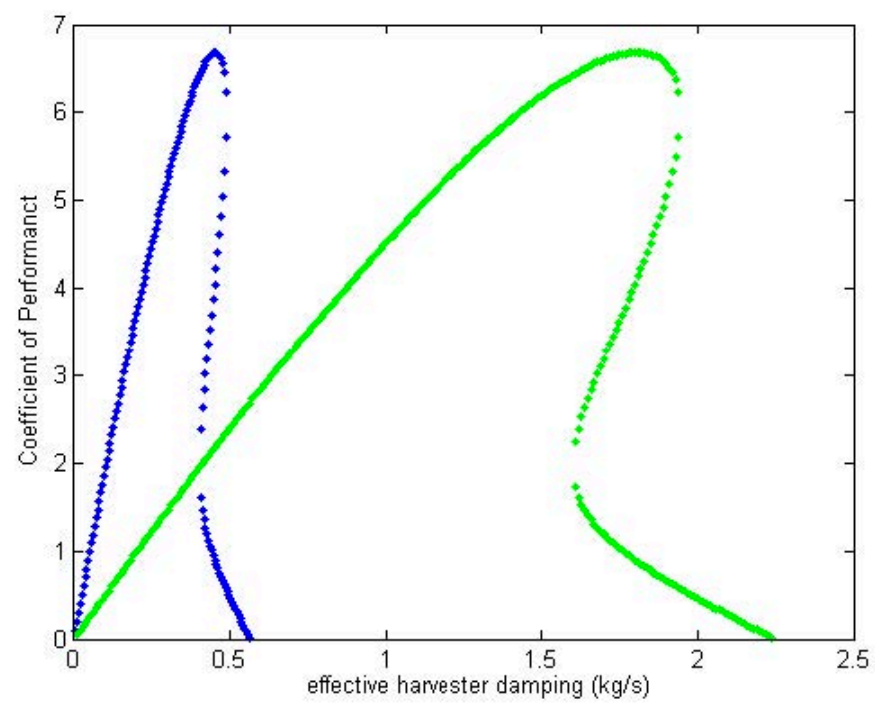

Figure HH - Coefficient of performance for a trapezoid. (blue) prism size 1” x 6”, (green) 2" x 12”. Flow velocity $2 \mathrm{ft} / \mathrm{s}$.

The equilateral triangle bluff body is likewise unusual in that it has a low projected coefficient of performance (see Figures JJ and KK).

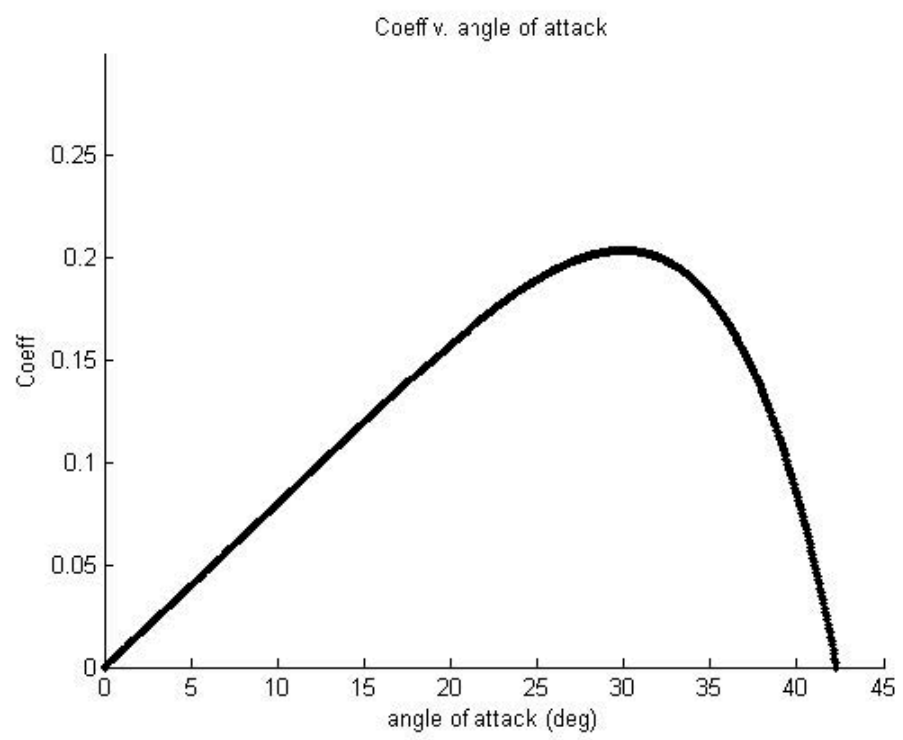

Figure JJ - Coefficient of lift for an equilateral triangle. Prism size 1” x 6”, fluid velocity $2 \mathrm{ft} / \mathrm{s}$. 


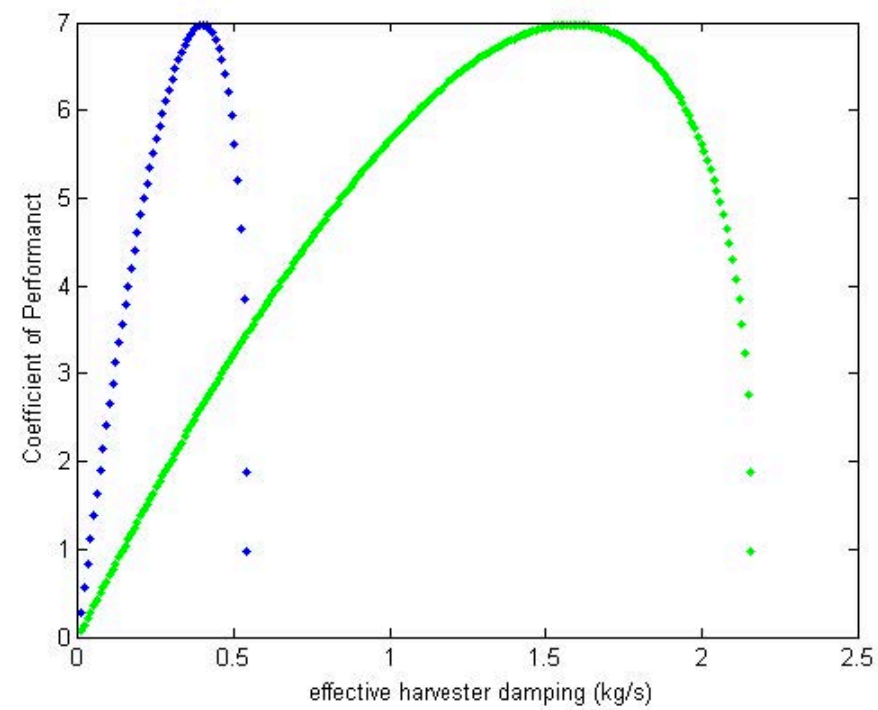

Figure KK - Coefficient of performance for a equilateral triangle. (blue) prism size 1" x 6”, (green) 2" x 12”. Flow velocity $2 \mathrm{ft} / \mathrm{s}$.

In fact, both the equilateral triangle and the trapezoid have very similar lift curves and thus similar performance evaluations.

As a final point of discussion, a comparison is made between the square and D-shape (see Figures LL and MM).

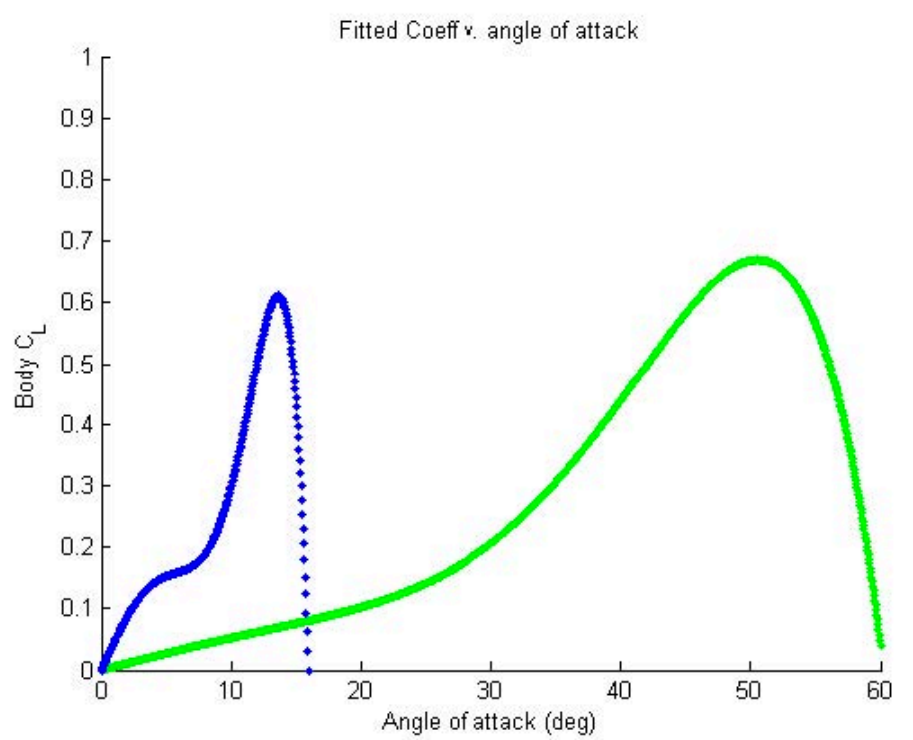

Figure LL - Coefficient of lift comparison between (blue) square-shaped and (green) D-shaped prisms. Prism size 1" x 6”. Fluid velocity $2 \mathrm{ft} / \mathrm{s}$. 


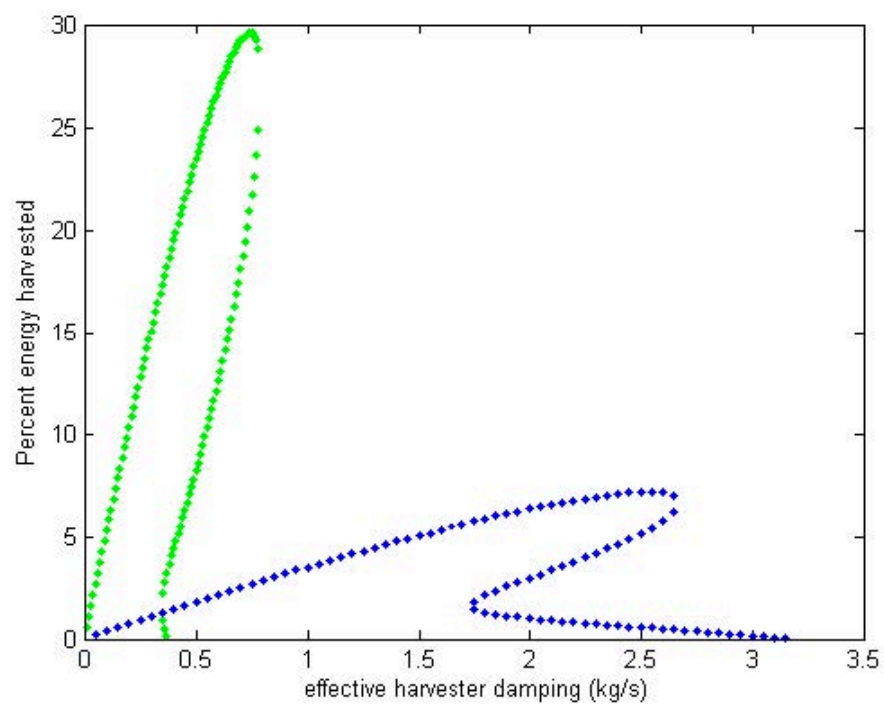

Figure MM - Coefficient of performance comparison between (blue) square-shaped and (green) D-shaped prisms. Prism size 1” x 6”. Fluid velocity $2 \mathrm{ft} / \mathrm{s}$.

The two curves have notably different characteristics, making advantages/disadvantages easier to note when compared together. The lift curve near the origin is steeper for the square than it is for the D-shape. This translates into a wider range of equivalentdamping due to energy harvesting for the square. The D-shape has a much larger range of angle of attack. Angle of attack is induced by relative velocity; so larger angle of attack indicates faster oscillation speeds, thus, greater RMS work per cycle for the Dshape than for the square.

\section{GHEED Generations}

Recall that the GHEED Generations portion of the program embodied two primary goals:

(1) Initiate the formation of a database of the relative performance of galloping prisms of varied geometry, size, and/or weight; and

(2) Create an alpha-phase experimental prototype of a GHEED in order to preliminarily assess the effectiveness of gallop as the primary physical mechanism for energy harvest.

Results and discussion of the database development efforts will be presented first, followed by that for GHEED prototype development.

\section{Database Development:}

Database development occurred in, essentially three phases. The first phase identified which prisms displayed gallop in irregular flows, and if so, the corresponding cut-in flow rate. As summarized in Tables 5 and 6, size, mass and surface finish are significant parameters in dynamic response. In addition, it is observed that for otherwise similar 
prisms, trailing edge effects play a major roll. It is also pertinent to note that all tested prisms were expected to display gallop in at least one orientation (based on reports from tests performed in laminar flows), but this proved not to be the case for ill-behaved flows.

\begin{tabular}{|c|c|c|c|}
\hline & Illustration & No Load Gallop? & $\begin{array}{l}\text { Electrically Loaded } \\
\text { Gallop? }\end{array}$ \\
\hline $\begin{array}{l}1 / 2 " \text { square in bluff-body } \\
\text { orientation }\end{array}$ & & No & \\
\hline $\begin{array}{l}1 / 2 " \text { square in diamond } \\
\text { orientation }\end{array}$ & & No & \\
\hline $1 / 2 " D$ & & No & \\
\hline $\begin{array}{l}1 \text { " Hex in low profile } \\
\text { orientation }\end{array}$ & & No & \\
\hline $\begin{array}{l}\text { 1" Hex with angled face } \\
\text { normal to flow }\end{array}$ & & No & \\
\hline $\begin{array}{l}\text { 1" Hex in bluff-body } \\
\text { orientation }\end{array}$ & & Yes & No \\
\hline $\begin{array}{l}\text { 1" square in bluff-body } \\
\text { orientation }\end{array}$ & & No & \\
\hline $\begin{array}{l}1 \text { " square in diamond } \\
\text { orientation }\end{array}$ & & Yes & Yes \\
\hline 1" D (38.6 g) & & Yes & Yes \\
\hline 1" Half-Tube & & Yes & Yes \\
\hline New 1" D (33.7 g) & & Yes & Yes \\
\hline $1 \frac{11 / 2}{11}$ & & Yes & Yes \\
\hline 1" Truncated D & & Yes & Yes \\
\hline $\begin{array}{l}45-45-90 \text { triangle with } 1 " \\
\text { legs }\end{array}$ & & Yes & Yes \\
\hline
\end{tabular}

TABLE 5 - Assessment of Whether Gallop Occurs For Varied Prism Shapes \& Orientations 


\begin{tabular}{|l|r|r|r|r|r|r|r|}
\hline & & & \multicolumn{4}{|l|}{ Gen-1 "Power" (mW) for flow rate of: } \\
\hline & mass (g) & Cut-in (m/s) & Cut-out (m/s) & $0.3 \mathrm{~m} / \mathrm{s}$ & $0.35 \mathrm{~m} / \mathrm{s}$ & $0.4 \mathrm{~m} / \mathrm{s}$ & $0.45 \mathrm{~m} / \mathrm{s}$ \\
\hline 1" tube & 26 & 0.3 & 0.55 & & 0.25 & 0.75 & 1 \\
\hline 1.5" D & 76 & 0.3 & & 0.4 & 0.5 & 0.4 & \\
\hline 45-45-90 & 43 & 0.3 & 0.5 & & 0.7 & 1.1 & 1.5 \\
\hline 1" trunc D & 30 & 0.3 & 0.45 & & 1.1 & 1.4 & 1.4 \\
\hline 1" new D & 34 & 0.3 & & & 1.1 & 1.4 & \\
\hline 1" orig D & 39 & 0.4 & & & & 0.8 & 1.1 \\
\hline
\end{tabular}

TABLE 6 - Relative Prism Performance using an EMI sensor attached to the Gen-1 GHEED

When considering the results of Table 6, it is important to recall that an EMI sensor (not a generator) was introduced to the Gen-1 device and used to project relative power performance. Thus, it is the relative response that is of interest, as opposed to the magnitude of response. These results were subsequently employed to down-select prisms worthy of characterization in the Gen-2 device.

Table 7 summarizes the subsequent results from prism testing in Gen-2, where the first column describes response at cut-in and the second column describes peak performance. In addition, an accelerometer was introduced to Gen-2; Figure NN illustrates a typical response. 


\begin{tabular}{|c|c|c|c|c|c|c|}
\hline & flow $(m / s)$ & Ampl. (mm) & Freq. $(\mathrm{Hz})$ & Flow $(\mathrm{m} / \mathrm{s})$ & Ampl (mm) & Freq $(\mathrm{Hz})$ \\
\hline $25 \mathrm{~mm} \mathrm{D}$ & 0.35 & 68 & 2.5 & 0.40 & 73 & 2.5 \\
\hline $33 \mathrm{~mm} \mathrm{D}$ & 0.35 & 73 & 2.3 & 0.40 & 73 & 2.5 \\
\hline $\begin{array}{l}25 \mathrm{~mm} \\
\text { Trapezoid }\end{array}$ & 0.30 & 23 & 1.6 & 0.55 & 45 & 1.9 \\
\hline $\begin{array}{l}24 \mathrm{~mm} \\
\text { Triangle }\end{array}$ & 0.30 & 27 & 1.9 & 0.55 & 56 & 2.2 \\
\hline $38 \mathrm{~mm}$ Rigid C & 0.30 & 20 & 1.4 & 0.55 & 51 & 1.6 \\
\hline "Morphed" C & 0.30 & 17 & 1.4 & 0.55 & 60 & 1.6 \\
\hline $\begin{array}{l}\text { Morphing } \\
\text { Run \#1 }\end{array}$ & 0.32 & 25 & 1.5 & 0.55 & 83 & 2.1 \\
\hline $\begin{array}{l}\text { Morphing } \\
\text { Run \#2 }\end{array}$ & 0.29 & 20 & 1.4 & 0.55 & 80 & 1.9 \\
\hline
\end{tabular}

Table 7 - Relative Prism Dynamic Performance in the Gen-2 fixture.

These results indicate that, of the rigid prisms, the D-shaped prism is the strongest performer over the range of testing while insertion of morphing enables earlier cut-in. It is also notable, that the frequency response for a given prism is relatively constant whereas the amplitude evolves; this was anticipated and therefore conforms with early assessments for power potential. From database generation initiated in this program it was concluded that a rigid-D shaped prism should be used as a frame of reference for all comparative analyses. (Discussion of the implications of morphing appears in the Morphing section of this report.) 

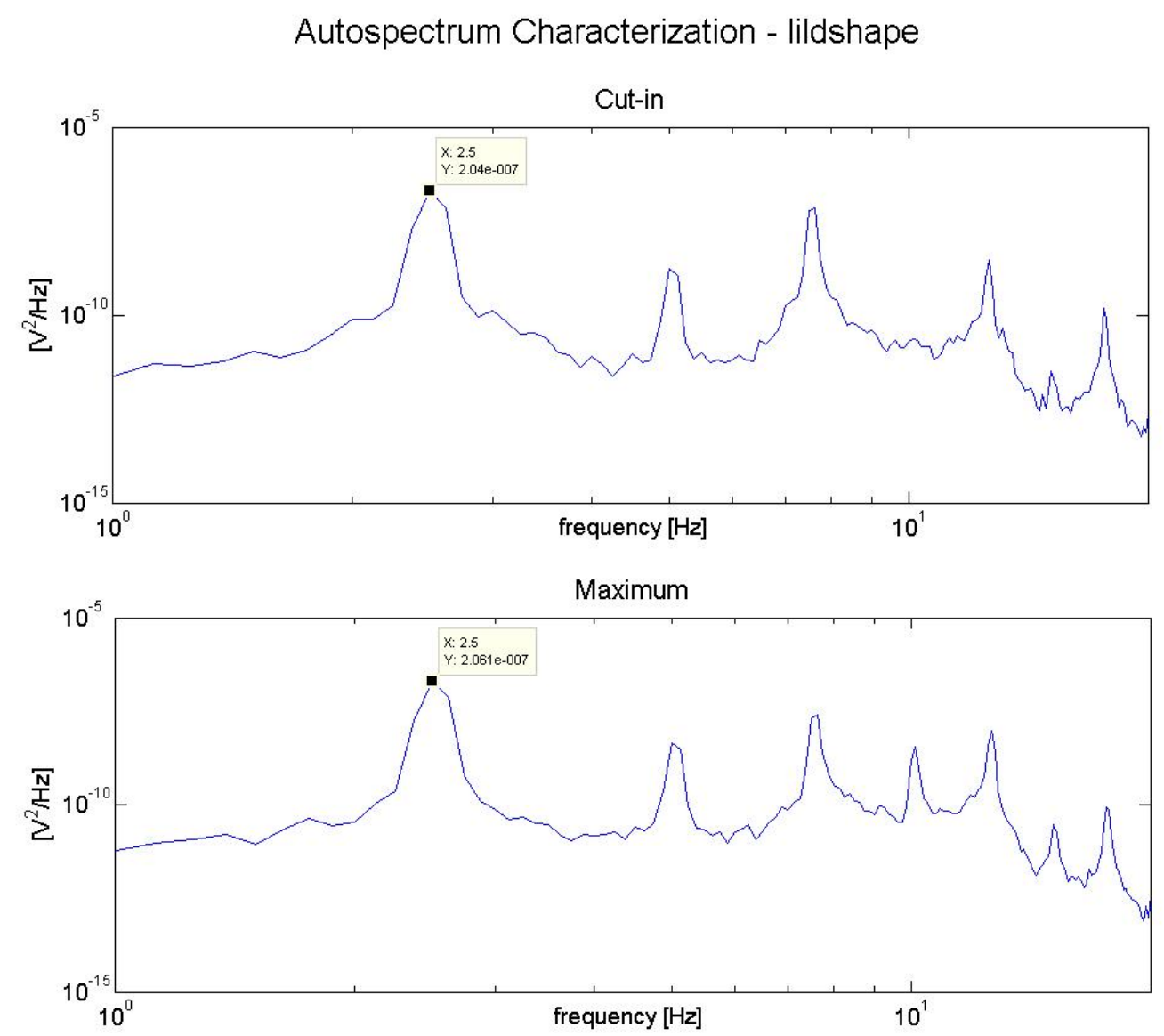

Figure NN- A typical autospectrum analysis used to assess frequency response. This image corresponds to the $25 \mathrm{~mm}$ rigid D-shaped prism.

\section{GHEED Design Evolution:}

Occurring in concert with database development was a redesign and rebuild of the GHEED test fixture. The decision to engage the rebuild was based on an unplanned, forcuriosity's-sake experiment where the Gen-1 GHEED was tested in a local stream. Instream performance of that device was far below that observed in the laboratory flume. Subsequently, application of the Gen-1 device was limited to preliminary down-selection of candidate prisms, as summarized above.

In developing Gen-2 it was concluded that the side-rail placement of the device against the flume walls had inadvertently created a forced flow diversion; the new design had a single, central rail and employed prisms which did not extend the full width of the flume to better emulate in-stream performance. The Gen-2 device included an accelerometer to assess gallop dynamics, where Figure NN offers a typical result, as well as the ability to vary the suspension. Figure PP illustrates the results of a test where the suspension was varied; the red line corresponds to a softer suspension. (Assessment of the mechanical power suggested that, while the dynamics were necessarily affected by the suspension, it 
did not appear that the available power had changed significantly.) While this type of assessment was useful for assessing gallop dynamics, it was concluded that a considerable number of assumptions were required for projecting the effect of generatorinduced damping on the gallop response. Initially ad hoc tests imposing mechanical damping were employed to better understand the implications of energy transduction; the results suggested that gallop dynamics would be diminished, but not necessarily damped out. However, because gallop is inherently nonlinear, it was concluded that an empirical approach would be preferred. Thus the design of Gen-3 introduced a generator.

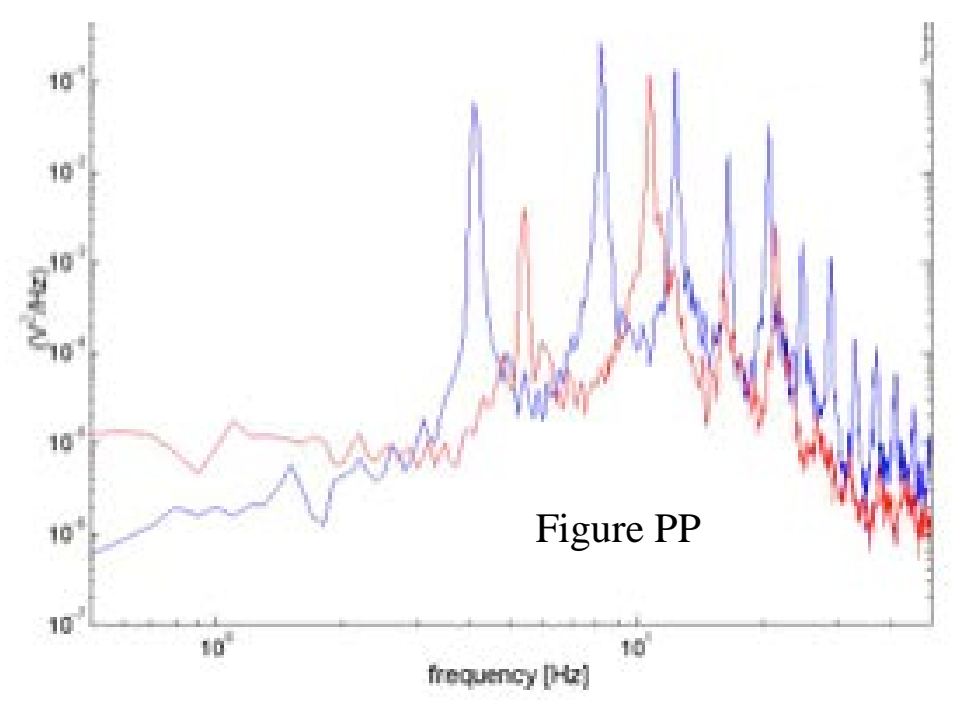

Figure PP - Effect of Varying Suspension Stiffness on Dynamic Gallop Response.

Mating a generator of any kind to a dynamic system is analogous to mating a damper to that system. A stronger generator will necessarily have stronger damping characteristics, and thus the dynamic motion will be diminished as electrical energy is increasingly extracted. While peak damping associated with a DEG will occur in a sense that is out of phase with peak damping from an EMI generator, and for that matter is expected to be more compatible with gallop dynamics than that of EMIs, developing some baseline for the effects of damping on gallop is of clear importance. Thus the next goal was to preliminarily characterize the trade-off between increasing electrical energy extraction and decreasing mechanical dynamics (or impedance matching); To assess this, the attached generator employed a variable air gap.

The results from the Gen-3 testing, per the protocol described in the Methods section of this document, are summarized in Figures QQ \& RR. The error bars within each figure establish strong repeatability for the test methodology, while comparing the two figures establishes appropriate repeatability for the Gen-3 design as a whole. 
In addition to Figure QQ \& RR drawing attention to the repeatability of both the testing methodology and the device itself, it is also important to note that the power harvest diminishes as electrical load decreases in each test - this indicates that appropriate communication had been established between the galloping oscillator and the EMI generator.
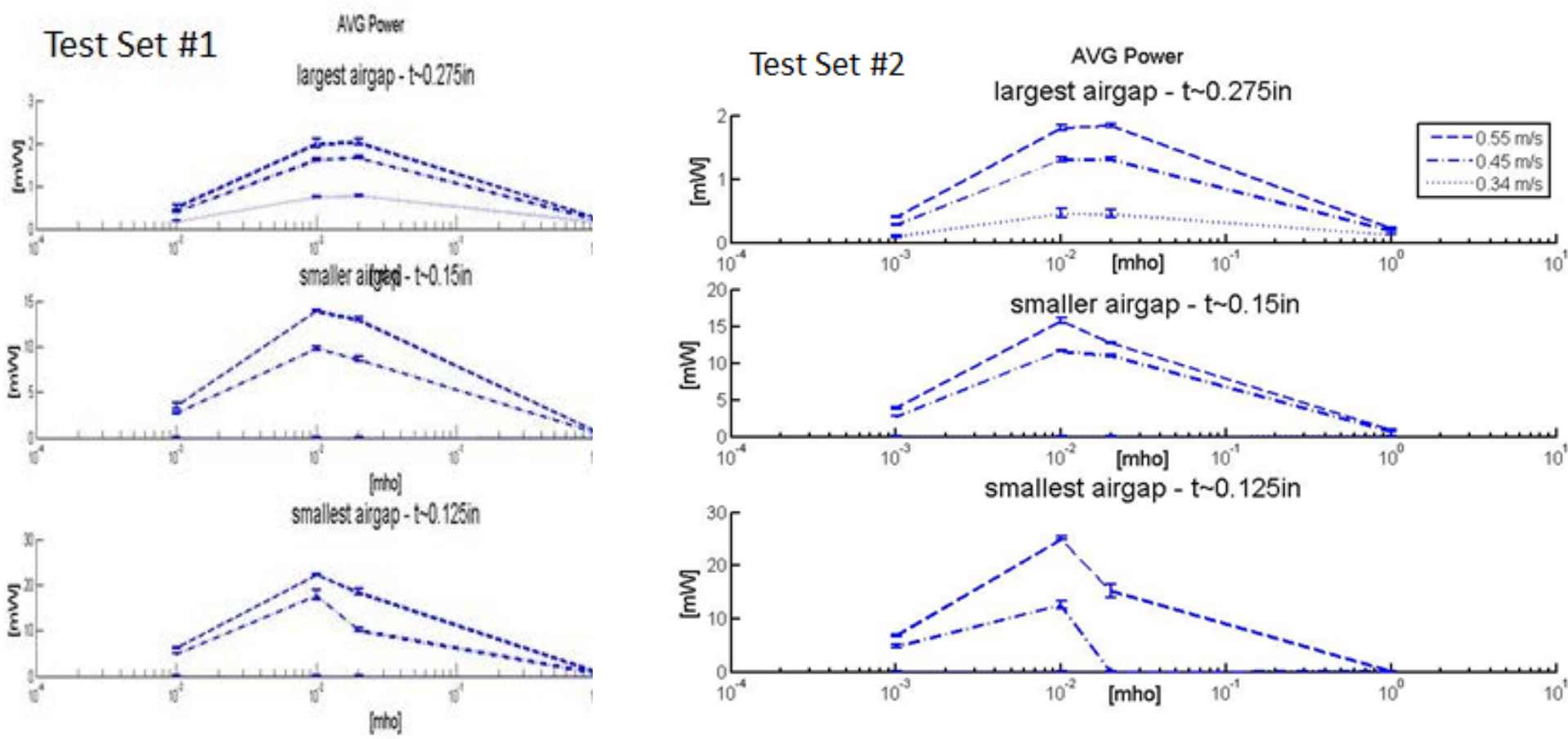

Figure QQ - (left) Evolution of power generation as a function of flow rate and air gap; each test performed at least 5 times to establish error bars, as illustrated.

Figure RR - (right) Repeatability test for evolution of power generation; the Gen-3 device was disassembled, reassembled, and then re-tested. This type of repeatability test is important because the gallop phenomenon is inherently unstable.

In order to assess the correlation between gallop dynamics and EMI-induced damping, the gallop dynamics were assessed via an accelerometer for each test. Figure SS offers an illustrative example of this assessment for a single test where the autospectrum response for acceleration has been converted into amplitude; Figure TT offers an illustrative example of how this assessment was used for comparative assessment. Figure SS illustrates example raw data (top) along with its integration so that gallop amplitude can be inferred. Figure TT illustrates the onset of diminished gallop amplitude as the EMI generator air gap is reduced, hence increasing power extraction as well as mechanical damping. Not illustrated, but of significance, as the air gap is reduced the cut-in speed is increased; air gaps smaller than $3 \mathrm{~mm}(0.125$ ”) displayed cut-in above that of the flume capacity. 

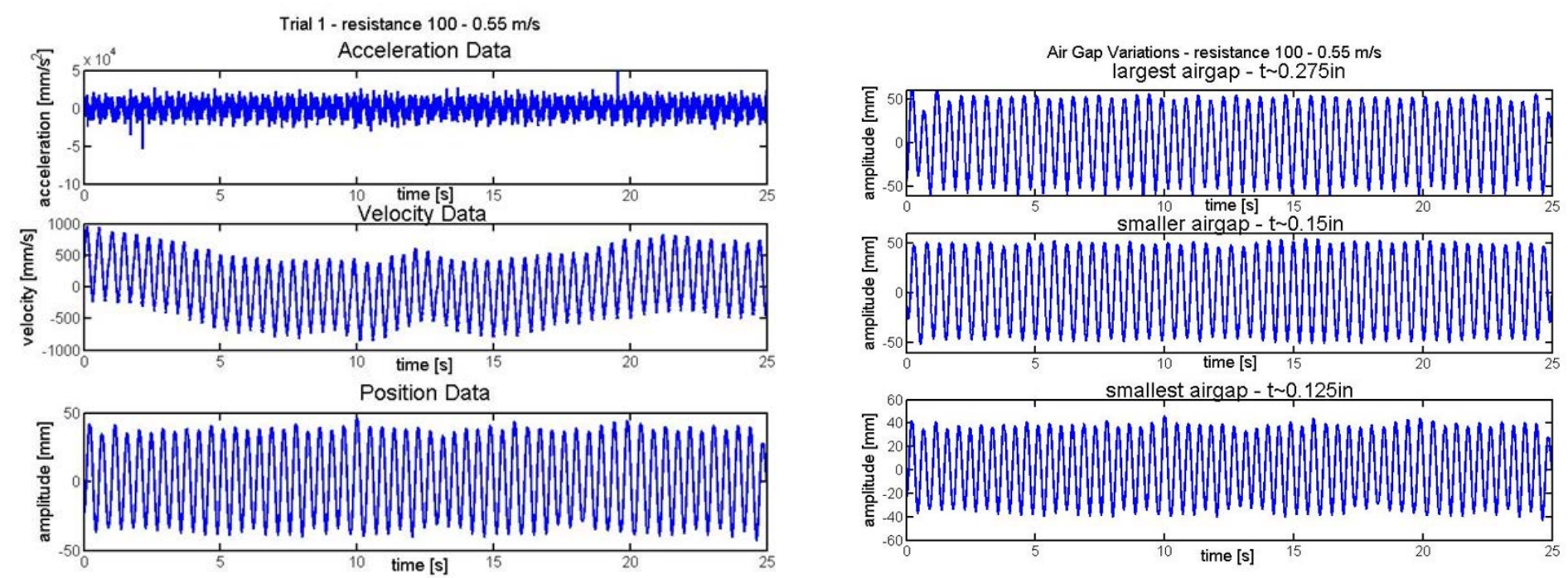

Figure SS - (left) Illustrative example of accelerometer data (top) and its integration (middle and bottom), enabling comparison of gallop dynamics among tests.

Figure TT - (right) Illustrative example of the resulting comparative assessments enabled. In this case it is observed that decreasing the generator air gap causes the galloping oscillation to display decreased amplitude due to damping.

In all, several hundred tests were performed, thus in the name of brevity only a few illustrative examples are offered. Other observations included the effects of varied flow rate and varied suspension stiffness: increased flow rate increased gallop amplitude but did not discernibly affect gallop frequency, while increased suspension stiffness reduced gallop amplitude while decreasing its frequency. Qualitatively, both of these results are consistent with expectation; their value resides in the quantification of the effect for this system.

Finally, Figure VV offers a summary of the entire battery of tests per the metric of power generation. As illustrated, as the air gap within the EMI generator is reduced, the power extracted is increased, but at the expense of increasing the cut-in speed. For instance, for a large air gap and soft suspension, cut-in is observed for flow rates as low as $0.34 \mathrm{~m} / \mathrm{s}$, while for reduced air gap and increased suspension cut-in does not occur until $0.55 \mathrm{~m} / \mathrm{s}$ (the peak flow rate of the flume), but this case also generates 50x to 60x more power. Because there is more power available for harvest at $0.55 \mathrm{~m} / \mathrm{s}$, it is prudent to also inspect these cases on the basis of the proportion available that has been harvested. On a Betz limit basis (which is not valid from a physics perspective, but still instructive on a benchmark basis), the test with very low cut-in harvested only about $0.25 \%$ of the available power, while the test case with elevated cut-in due to the decreased air gap 
harvested almost $4 \%$. Thus even from this basis the smaller air gap case outperforms the larger air gap case by more than an order of magnitude. The fact that even the improved case is too low is attributed to the limits of testing available; in other words, the tests presented were performed up to the performance limits of the flume (max flow speed of $0.55 \mathrm{~m} / \mathrm{s}$ ); however the trend observed from these tests suggests that cutting the air gap by another 4-fold, which is still quite large by generator standards, would increase the cut-in speed to about $1 \mathrm{~m} / \mathrm{s}$ while also increasing the harvest effectiveness to 65\%-70\%. Thus, for the current state of development, appreciation of the cut-in power trade-off is useful from a design-to perspective, but it is of course the elevated cases that will ultimately warrant dedicated attention.

Regarding the specific effect of varied suspension, the results of Figure VV are suggestive that a stiffer suspension may tend toward higher power generation; hence a stiffer suspension may be desirable. But potentially more important, and as noted above, the amplitude of oscillation decreases as the suspension becomes stiffer. Thus, even if power generation was observed to hold steady as suspension evolves, a stiffer suspension is again favored because a smaller swept area in application will be desirable. This observation is important both from the view point of optimizing power production in the general sense, but also from the view point of mapping a strategy for coupling a DEG to the gallop device since the DEG will double as the suspension.

In sum, the experimental results from the Gen-3 GHEED offer guidance for coupling a DEG to the gallop device in that we now quantifiably understand: (i) a somewhat stiffer DEG suspension will be desirable, with that stiffness driven primarily by the sought swept area, and (ii) how enhancing the mating between the gallop device and the generator simultaneously elevate cut-in speed and energy harvest effectiveness. These results can subsequently be used to guide the preferred mating of the galloping mechanical system to the DEG electrical system. 

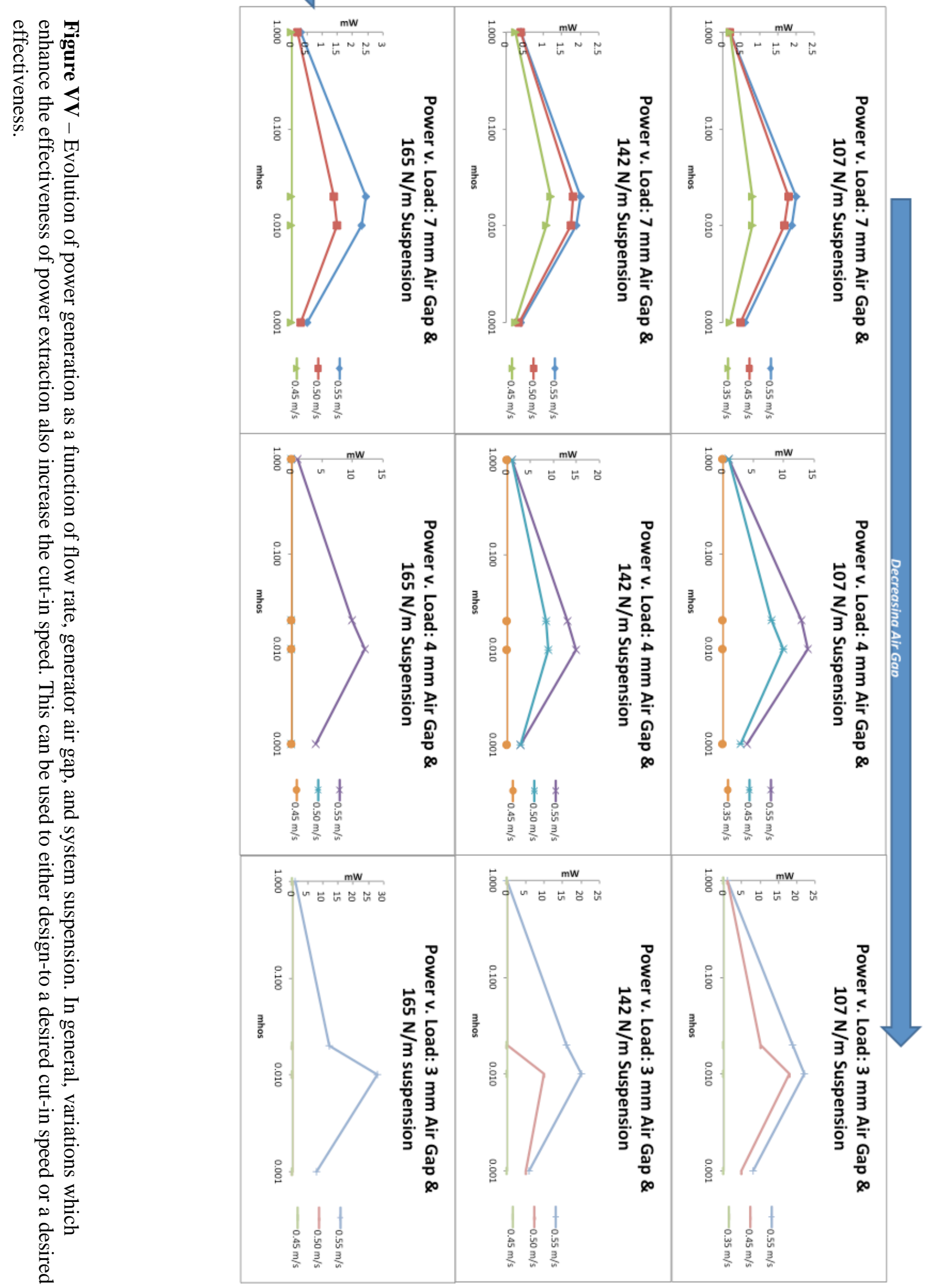


\section{Morphing}

Recall the primary goals of the morphing effort were to establish that (i) morphing could be used to exert control over gallop response, and (ii) that a morphing design strategy could be developed.

The morphing body design methodology begins by adapting a traditional analytical modeling approach for isotropic homogenous curved bodies to predicting passive deformation of an anisotropic morphing body. Namely, Castigliano's Theorem for curved bodies is adapted, where the resulting expression is as follows:

$$
\begin{aligned}
\delta_{\text {tot }}=\left\{\frac{p R_{T}^{3} l}{A_{T} e_{T} E_{T}}-\frac{p R_{T}^{2} l}{A_{T} E_{T}}\right\}\left\{\frac{2}{3} \sin ^{3} \theta_{t r}-\frac{1}{3} \sin ^{3} \theta_{r e l}\right\}+\left\{\frac{p R_{C}^{3} l}{A_{C} e_{C} E_{C}}-\frac{p R_{C}^{2} l}{A_{C} E_{C}}\right\}\left\{\frac{2}{3}-\frac{2}{3} \sin ^{3} \theta_{t r}\right\} \\
+\left\{\frac{p C R_{T}^{2} l}{3 A_{T} G_{T}}\right\}\left\{2 \cos ^{2} \theta_{t r} \sin \theta_{t r}+4 \sin \theta_{t r}-\cos ^{2} \theta_{r e l} \sin \theta_{r e l}-2 \sin \theta_{r e l}\right\}+\left\{\frac{p C R_{C}^{2} l}{\left.3 A_{C} G_{C}\right\}}\left\{2-\cos ^{2} \theta_{t r} \sin \theta_{t r}-2 \sin \theta_{t r}\right\}\right. \\
\qquad \\
\delta_{\text {tot }}=2 \delta
\end{aligned}
$$

In this expression, the subscripts $\mathrm{T}$ and $\mathrm{C}$ represent 'tips' and 'center' respectively, and the transition angle is that at which the material and/or thickness shifts. (Ultimately some form of functional gradation will be warranted to avoid stress concentrations, but this approach is effective for the desired purpose.)

Using the expression, two morphing C-shapes were designed and tested as summarized in Figures WW, XX and YY. Each test was repeated at least 5 times; the data sets presented are illustrative examples. As seen in Figure YY, the design model corresponded reasonably well with experiment, especially for the larger of the morphing prisms. In light of the ease of use of the design model, the margin of error between model and experiment was deemed acceptable. 


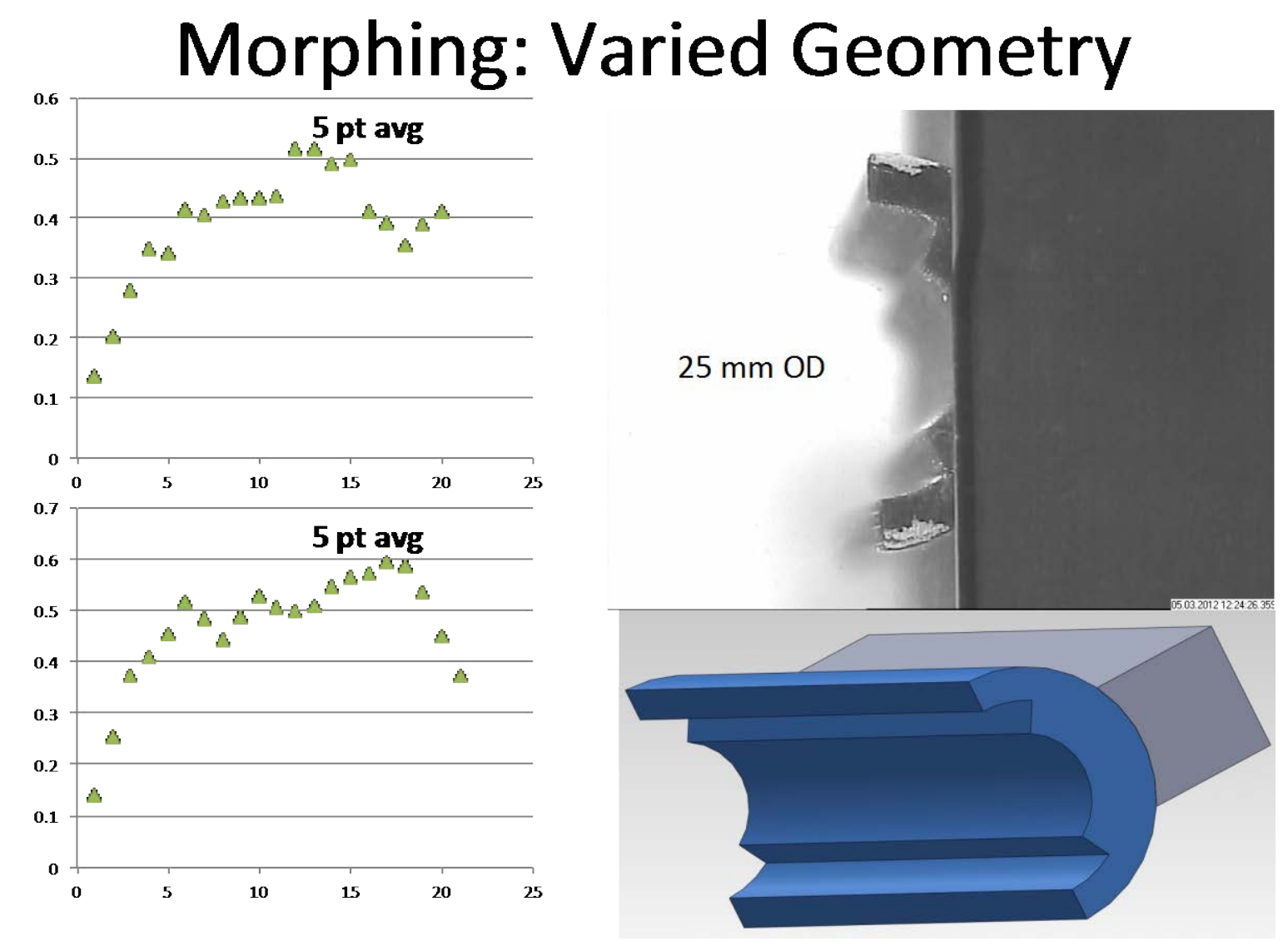

Figure WW - (Bottom Right) Design of a 25 mm outer diameter passively morphing prism where varied material thickness affects the desired flaring response to fluid pressure. (Top Right) Submerged morphing prism during testing where white marks at tips enable a video extensometer to monitor tip opening. (Left)

Raw data for two representative tests; ordinate (y-axis) is engineering strain observed by the video extensometer; abscissa (x-axis) is time which arbitrarily illustrates increasing flow rate with time - the peak strain correlates with peak flow rate of $0.55 \mathrm{~m} / \mathrm{s}$. 


\section{Morphing: Varied Material}
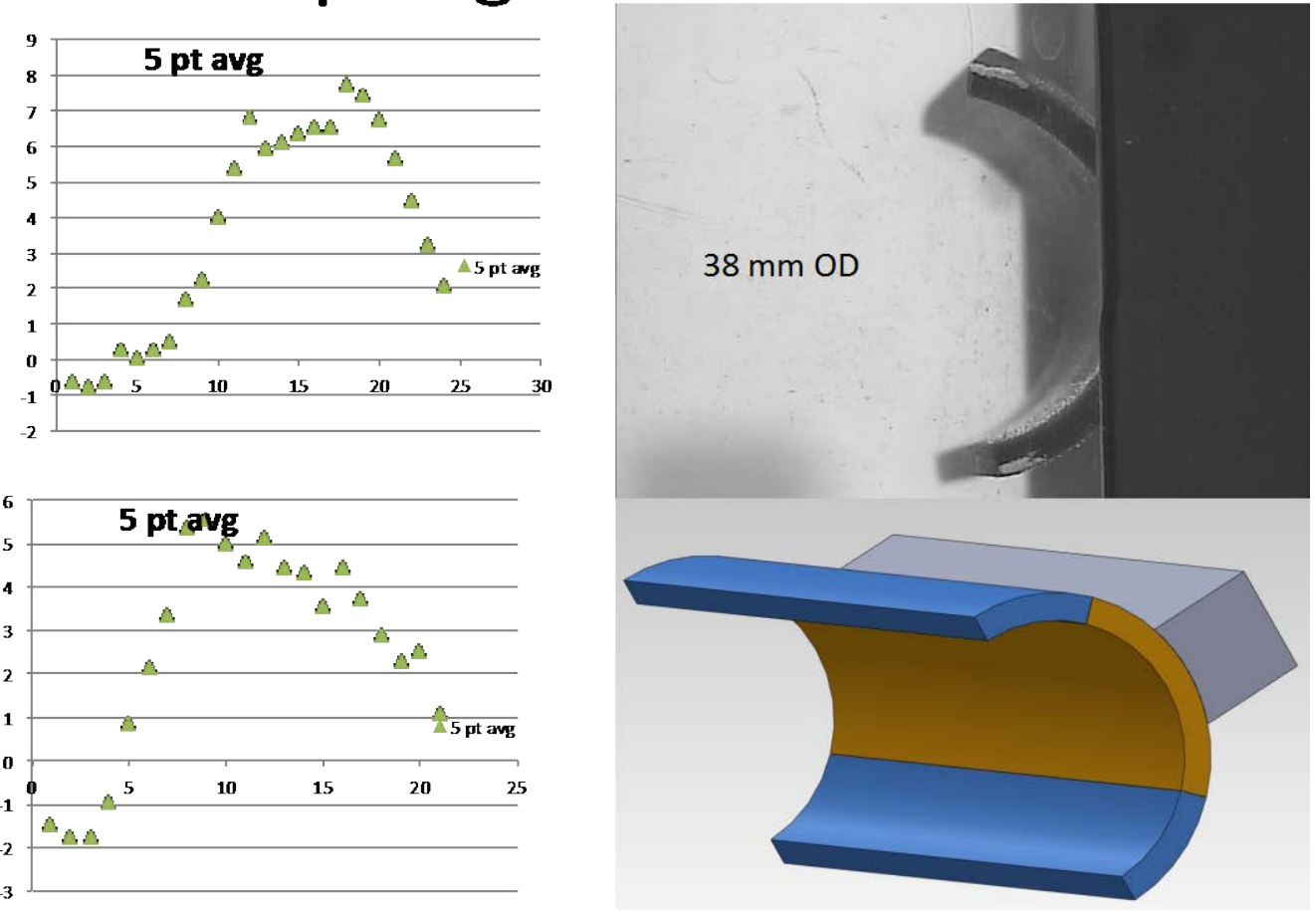

Figure XX - (Bottom Right) Design of a $38 \mathrm{~mm}$ outer diameter passively morphing prism where varied material type affects the desired flaring response to fluid pressure. (Top Right) Submerged morphing prism during testing where white marks at tips enable a video extensometer to monitor tip opening. (Left) Raw data for two representative tests; ordinate (y-axis) is engineering strain observed by the video extensometer; abscissa (x-axis) is time which arbitrarily illustrates increasing flow rate with time - the peak strain correlate with the peak flow rate of $0.55 \mathrm{~m} / \mathrm{s}$. 


\section{Morphing: Comparison to Model?}

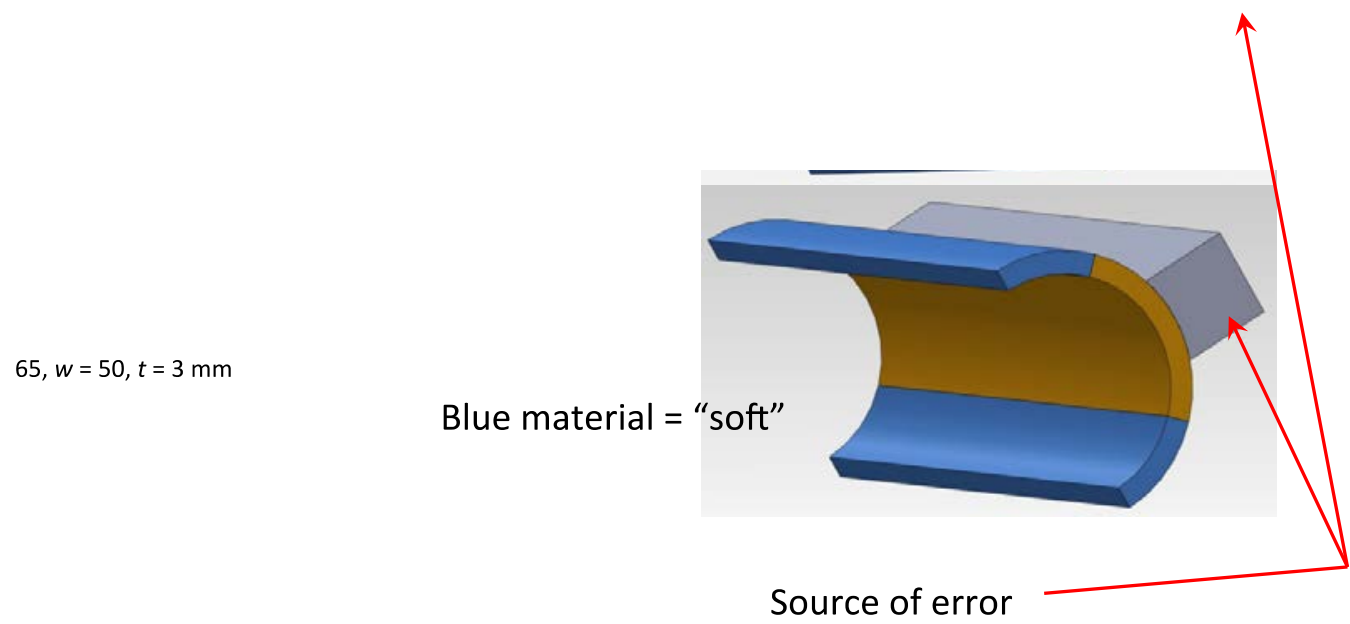

Figure YY -(Right) Design of a $25 \mathrm{~mm}$ outer diameter varied thickness passively morphing prism and a $38 \mathrm{~mm}$ outer diameter varied material morphing prism. (Bottom) The expression employed to predict morphing deformation. (Left) Conversion of raw experimental data for each prism into tip opening in comparison to prediction.

The transition to dynamic testing then explored the predicted deformation for each of the prisms if the angle of attack is varied (the linear galloping oscillation necessarily results in relative motion that effectively varies the angle of attack). Per the same methods as above, the prediction was compared to experiment. The two were in similar agreement, and both suggested that the variation in tip opening due to oscillation was small compared to the overall tip opening, and could therefore be neglected.

The larger of the prisms was then selected for dynamic testing in the galloping fixtures, in part because the morphing was pronounced and would therefore enable clear inspection (beyond the error bars) of the effect. (It is important to emphasize that the specific demonstration candidate selected, while it did ultimately display intriguing response in addition to demonstrating the primary goals of this phase of work, is not likely to represent an idealized morphing candidate.)

As summarized in Table 7 of the previous section, application of the morphing prism had two notable potential benefits. First, the range of operation was expanded (lower cut-in flow rate) as compared to the best performing rigid prism. But in light of the fact that it is the methodology that is of interest, what is more noteworthy is that the morphing prism 
outperformed each of its rigid counterparts. In other words, it performed at an even higher level than simply retaining the best of each of the rigid extremes, thus adaptive response favored enhance response. This was naturally intriguing, and therefore warranted additional inspection.

Figure ZZ below illustrates that the enhanced dynamic response does indeed carry over into enhanced power potential as compared to the rigid counterparts. But more importantly, these results demonstrate that shape morphing can be employed to meaningfully affect GHEED energy harvest, and that a straight forward design methodology can be invoked in its design. Moving forward (post program) the issue becomes one of clearly defining which aspect of GHEED energy harvest is targeted for optimization. For instance, for applications with widely varying flow rates it may be desirable to invoke morphing to expand the range over which galloping energy harvest occurs, such as that which as has been demonstrated here. Alternatively, morphing could be invoked to enhance the harvest over a specific flow range, so that the system is tuned to optimum performance in that range. 

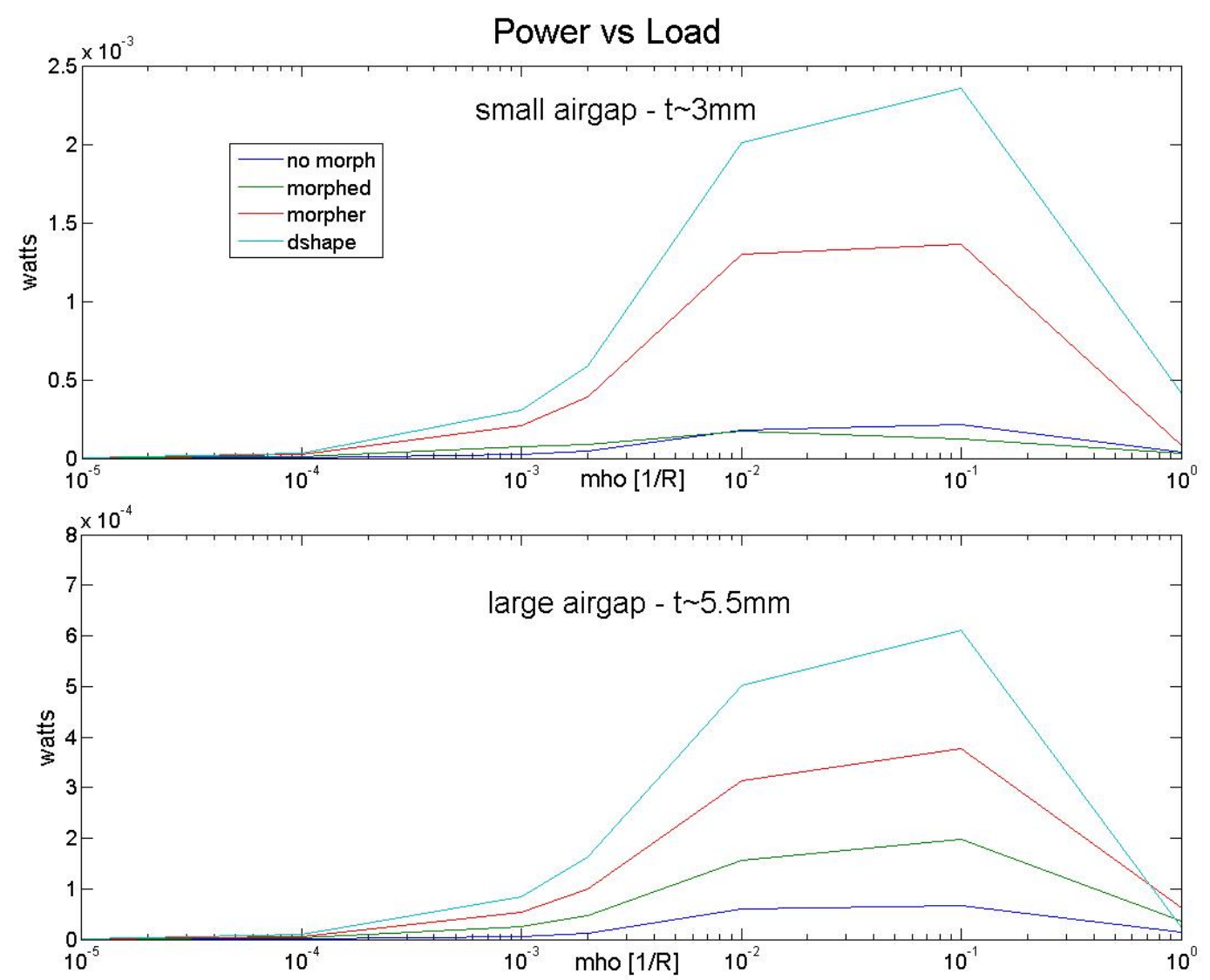

Figure ZZ - Test fixture data for the rigid C- shape ("no morph," also described as 'unflared'), the rigid morphed C-shape ("morphed," also described as 'flared'), the morphing C-shape (“morpher”), and the rigid D-shape prism under different generator air gaps and flume flow velocities. In addition to illustrating the superior performance of the morphing prism over its rigid counterparts, the figure also illustrates the methodology required to impedance match a generator to gallop, though in this case the air gaps were too big to generate interesting power.

\section{DEG Testing}

\section{Single DEG Simulation and Calibration to Data:}

The following section gives a detailed account of the mathematics behind the single DEG simulation. As mentioned before, this simulation assumes a sinusoidal velocity source and that the input parameters, namely $x_{\max }, x_{\min }, V_{\text {seed }}, R_{E L}, f$, and $N$, are known. Since the simulation is a numerical model in the time domain, the first variable to define is the time step which is the length of time between data points,

$$
\Delta t=\frac{1}{1000 f}
$$


The time step is normalized to the frequency such that there are 1000 data points per cycle. We use this time step to define the time array $(t)$ which starts at 0 seconds, ends at $\frac{N}{f}$ seconds and increments in steps of $\Delta t$ seconds. Next we define $A$ to be the amplitude of the oscillations which is given by,

$$
A=\frac{1}{2}\left(x_{\max }-x_{\min }\right)
$$

The velocity $(u)$ is defined such that the displacement $(x)$ will oscillate between $x_{\max }$ and $x_{\min }$,

$$
u(t)=-2 \pi f A \sin (2 \pi f t)
$$

Which, when integrated gives the displacement,

$$
x(t)=\int u(t) d t=A \cos (2 \pi f t)+D
$$

To find the constant of integration $(D)$, we use the initial condition $x(0)=x_{\max }$, therefore,

$$
\begin{gathered}
x(0)=A \cos (0)+D \\
x_{\max }=A+D \\
D=x_{\max }-A
\end{gathered}
$$

The equation for displacement is then,

$$
x(t)=A \cos (2 \pi f t)+x_{\max }-A
$$

The equations for the capacitance $(C)$ and the elastic force $\left(F_{S}\right)$ are both third order polynomials which were fitted to the $0.175 \mathrm{~Hz}, 500 \mathrm{~V}$ SRI data sets using a least squares fitting program. The coefficients of the polynomials depend on whether the simulation is for the 2 element or the 4 element case. The capacitance equation was fitted so that the capacitance is zero when $x=0$,

$$
\begin{gathered}
F_{S}(x)=\beta_{3} x^{3}+\beta_{2} x^{2}+\beta_{1} x+\beta_{0} \\
C(x)=\alpha_{3} x^{3}+\alpha_{2} x^{2}+\alpha_{1} x
\end{gathered}
$$

The following graphs show the force data and capacitance data for the 4 element, 0.175 $\mathrm{Hz}, 500 \mathrm{~V}$ data set as well as the polynomial fits used to approximate the elastic force and the capacitance. 


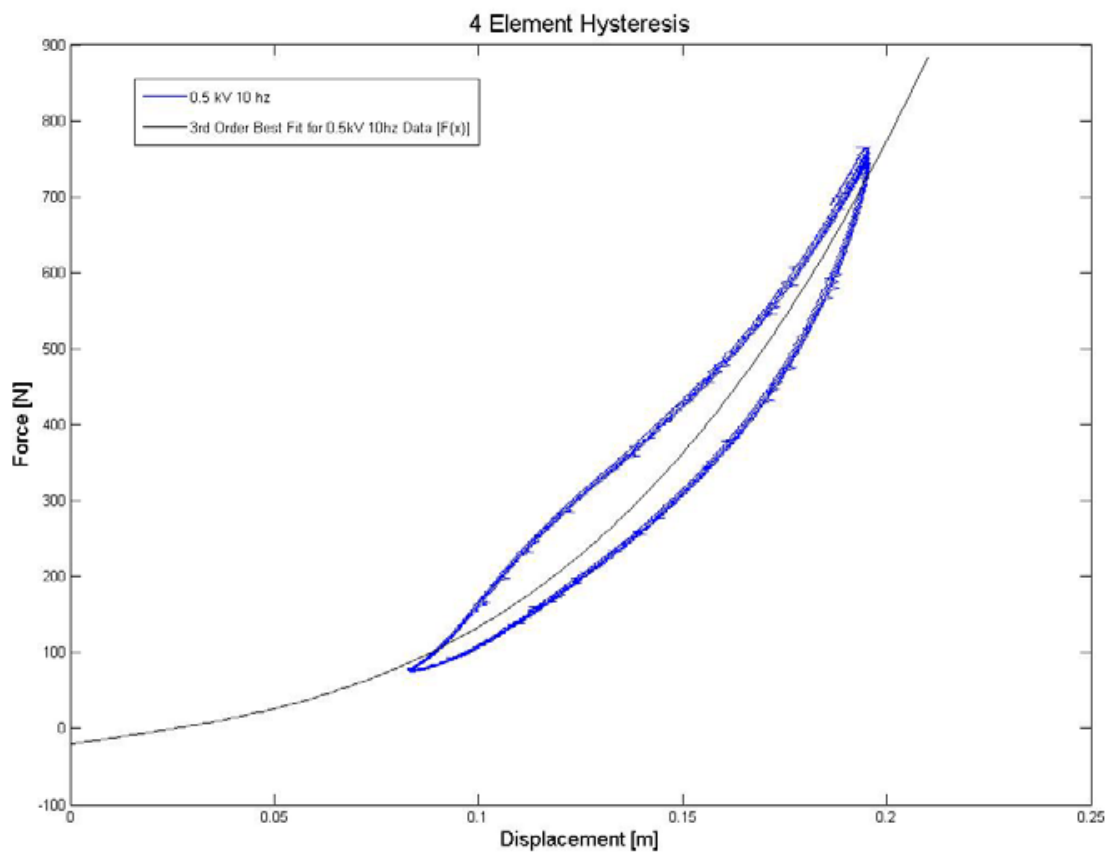

Figure AB - Force graph for a 4 element single DEG.

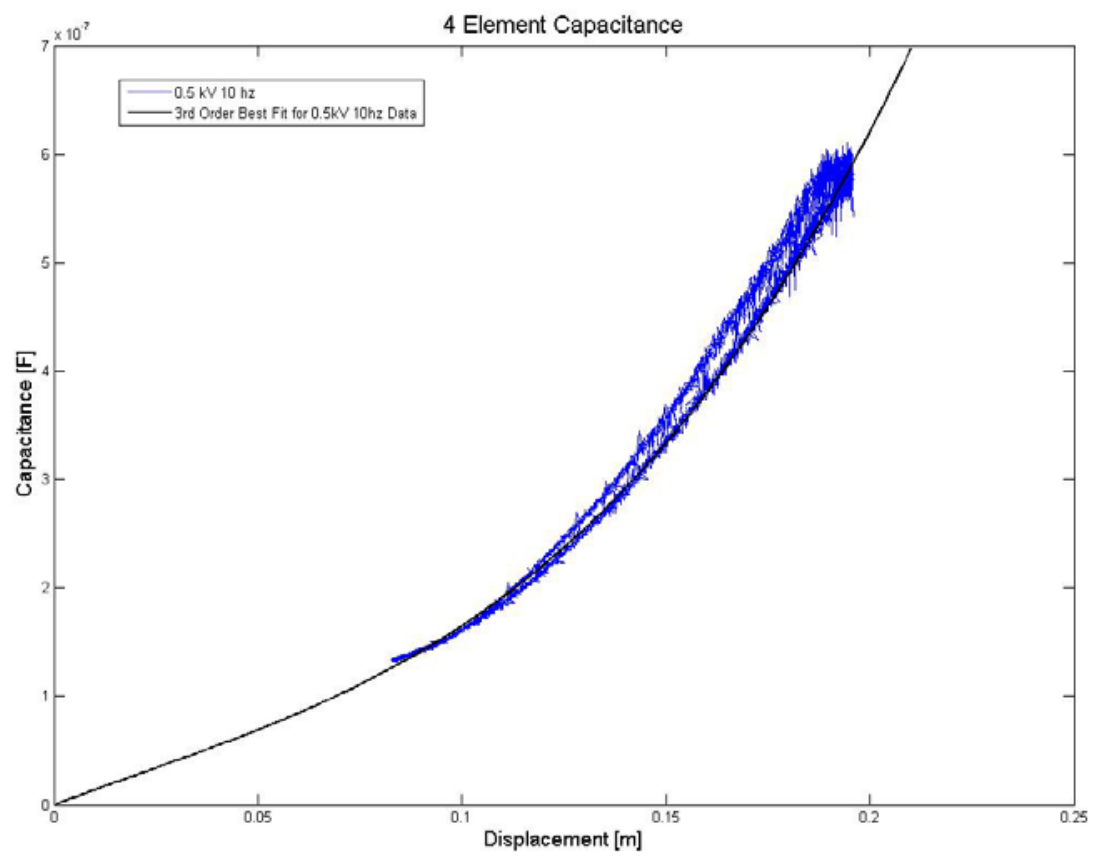

Figure AC - Capacitance graph for a 4 element single DEG. 
As mentioned before, the maximum and minimum values of the capacitance occur at the maximum and minimum displacements respectively. Therefore the equation for capacitance can be used to obtain $C_{\max }$ and $C_{\min }$,

$$
C_{\max }=C\left(x_{\max }\right) \text { and } C_{\min }=C\left(x_{\min }\right)
$$

Since the seed voltage is applied to the film when the displacement has reached $x_{\max }$ this means that the corresponding capacitance will be $C_{\text {max }}$. Therefore the seed charge on the acrylic film can be calculated,

$$
Q_{\text {seed }}=C_{\text {max }} V_{\text {seed }}
$$

The tests done at SRI and the simulations generated at AMI use the constant charge method for applying voltage to the film. Thus for an ideal capacitor the charge on the film would remain constant throughout the entire relaxation section of the cycle.

However, in practice there are electrical losses in the capacitor that are accounted for by simulating leakage current though the acrylic film. The leakage current is represented by a resistor $\left(R_{E L}\right)$ in parallel with the capacitor and the leaked charge is introduced using the finite difference method,

$$
\begin{gathered}
Q\left(x_{i+1}\right)=Q\left(x_{i}\right)-\frac{V\left(x_{i}\right)}{R_{E L}} \Delta t \\
V\left(x_{i+1}\right)=\frac{Q\left(x_{i+1}\right)}{C\left(x_{i+1}\right)}
\end{gathered}
$$

The above equations for voltage and charge only apply when voltage is applied to the film (i.e. when the film is being relaxed from its maximum to its minimum displacement). When the film is being stretched the charge and the voltage are both zero. The equation for voltage can be used to find the maximum voltage $\left(V_{\max }\right)$ which occurs at the minimum displacement, therefore,

$$
V_{\max }=V\left(x_{\min }\right)
$$

The maximum voltage will be used later when calculating the energy harvested per cycle. Mechanical losses are represented by viscous damping where the damping term $\left(c_{M L}\right)$ is a function of the RMS velocity $\left(u_{R M S}\right)$. The equation for the damping term and the damping force $\left(F_{c}\right)$ are given by,

$$
\begin{gathered}
c_{M L}=\delta\left(u_{R M S}\right)^{\tau} \\
F_{c}=c_{M L} u
\end{gathered}
$$

Where the values for $\delta$ and $\tau$ depend on whether the simulation is the 4 element or the 2 element case and these values were calculated using a least absolute deviation fitting program. The graphs below shows the damping term data points that were calculated in the analysis and the fit that is used to generate the values for $\delta$ and $\tau$. 


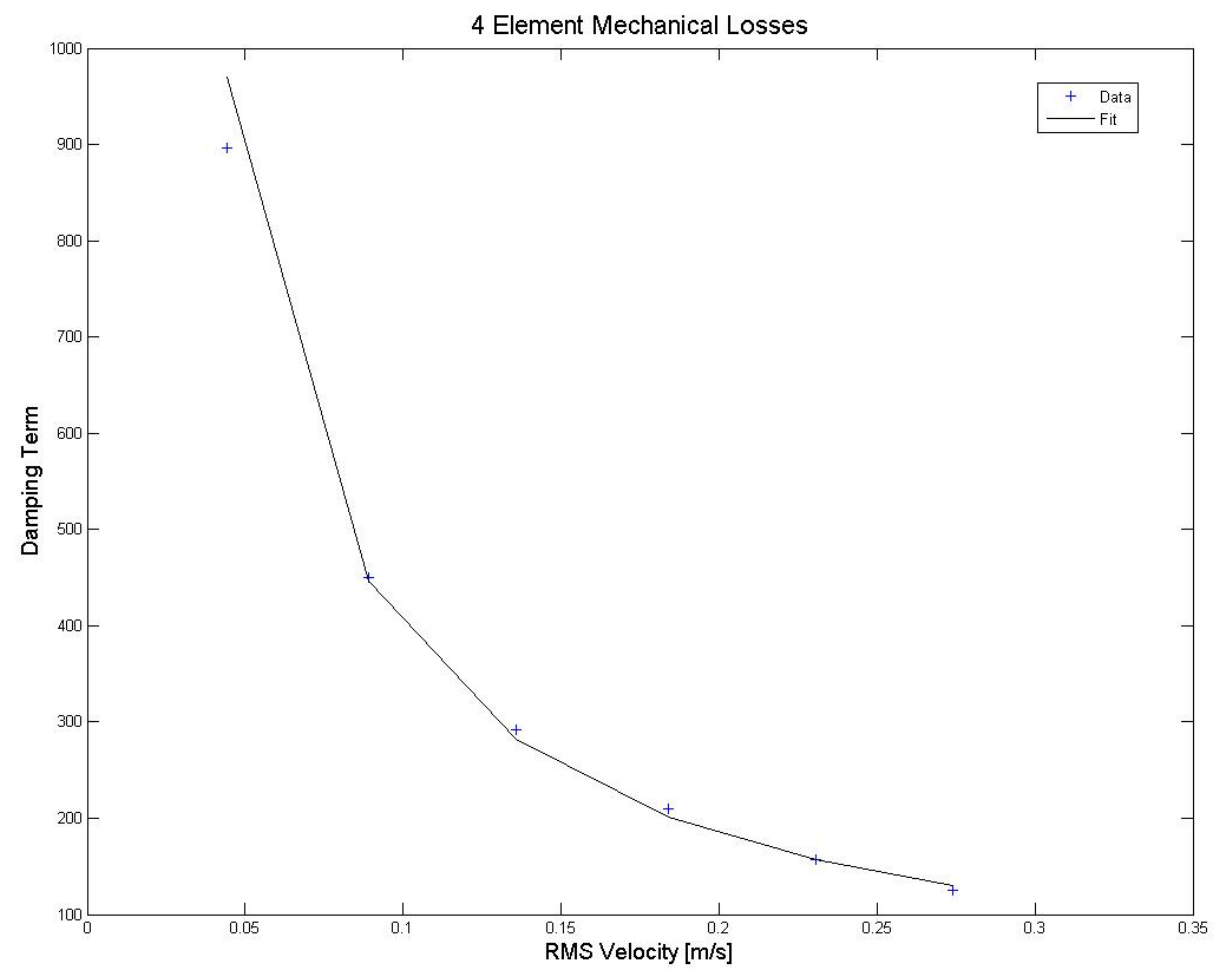

Figure AD - Mechanical losses and data fit for a 4 element single DEG.

The next force to account for is the electrostatic force $\left(F_{E}\right)$ which is given by the following equation,

$$
F_{E}=\frac{1}{2} \frac{d C}{d x} V^{2}
$$

The mechanical force $\left(F_{M}\right)$ is defined as the interaction of the elastic force, damping force and the electrostatic force. Therefore the mechanical force is given by:

$$
F_{M}=F_{S}+F_{c}-F_{E}
$$

The various force are shown for the 4 element case in the following graph, 


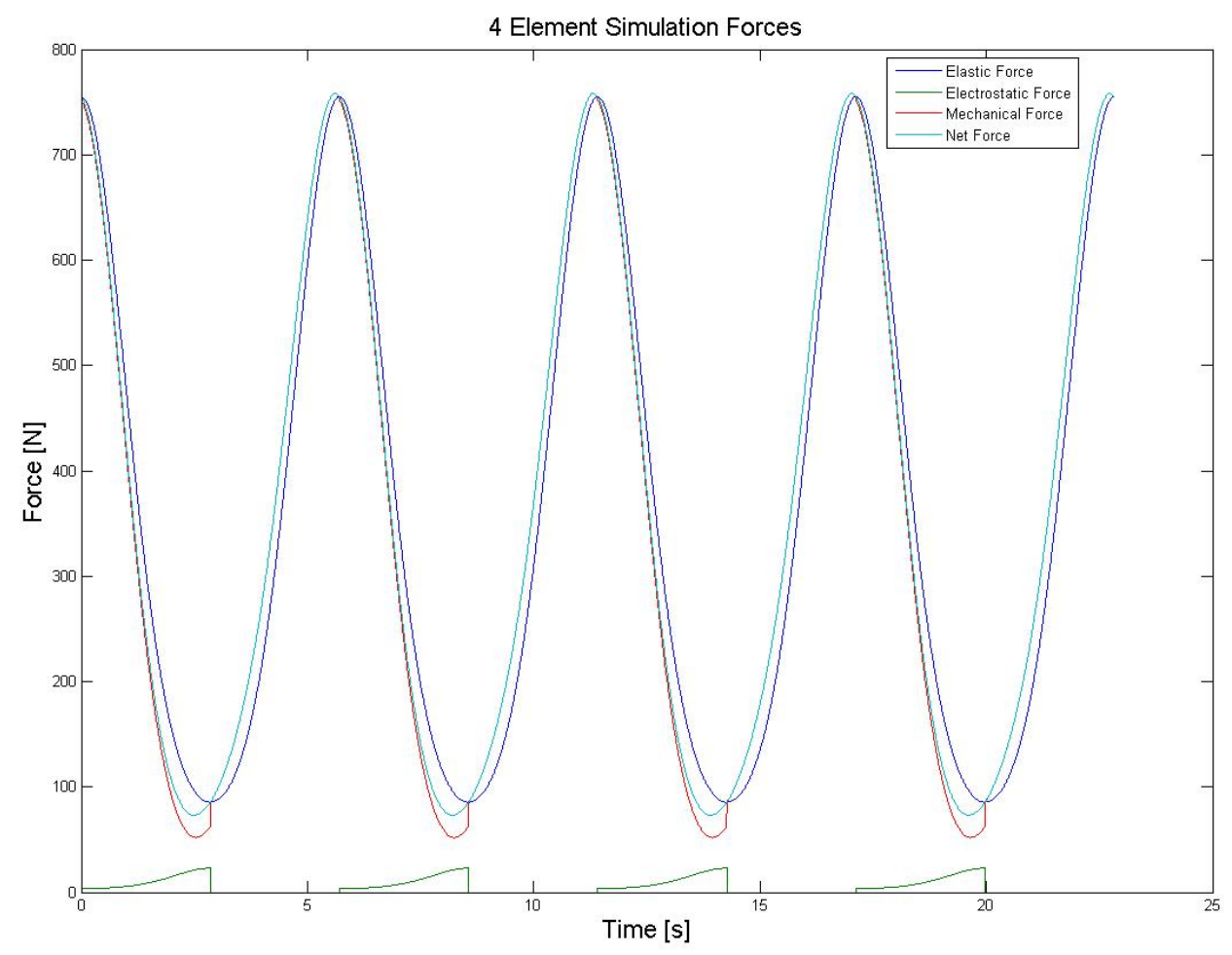

Figure AE - Various forces for a 4 element single DEG.

The last part of the simulation is the calculation of the energy terms of which there are five: $W_{E L}, W_{M L}, W_{E}, W_{M}, W_{H}$. The variables $W_{E L}$ and $W_{M L}$ represent the average energy loss per cycle due to electrical and mechanical losses respectively. The next two variables, $W_{E}$ and $W_{M}$, represent the electric potential energy (usually denoted $U$ ) and the instantaneous mechanical energy respectively. The last variable, $W_{H}$, is the energy harvested per cycle.

The average energy loss per cycle due to electrical losses is calculated by integrating the power dissipated across $R_{E L}$ and then dividing by the total number of cycles. Similarly, the average energy loss per cycle due to mechanical losses is calculated by integrating the mechanical power and then dividing by the total number of cycles.

$$
\begin{gathered}
W_{E L}=\frac{\int V^{2} d t}{R_{E L} N} \\
W_{M L}=\frac{\int F_{M} v d t}{N}
\end{gathered}
$$

The electric potential energy stored on the film and instantaneous mechanical energy are given below:

$$
W_{E}=\frac{1}{2} C V^{2}
$$




$$
W_{M}\left(t_{i}\right)=\int_{0}^{t_{i}} F_{M} v d t
$$

The energy harvested per cycle is equal to the difference between the electric potential energy stored on the film at the minimum and maximum displacements.

$$
W_{H}=\frac{1}{2}\left(C_{\min } V_{\max }^{2}-C_{\max } V_{\text {seed }}^{2}\right)
$$

This concludes the description of the equations and theory for a single DEG with energy harvesting.

\section{Coupled DEG Simulation:}

The implementation of the coupled DEG simulation is fairly straightforward since it involves the combination of two single DEGs with energy harvesting where the DEGs are 180 degrees out of phase with each other. Thus the velocity of the first DEG is given by,

$$
u_{1}(t)=-2 \pi f A \sin (2 \pi f t)
$$

And the velocity of the second DEG is given by,

$$
u_{2}(t)=-2 \pi f A \sin (2 \pi f t+\pi)
$$

The elastic forces for DEG $1\left(F_{S 1}\right)$ and DEG $2\left(F_{S 2}\right)$ are then added together resulting in the net elastic force:

$$
F_{S 1+S 2}=F_{S 1}+\left(-F_{S 2}\right)
$$

The negative accounts for the difference in direction. For the 4 element case, it turns out that even though $F_{S 1}$ and $F_{S 2}$ are nonlinear, their sum is linear and can be very closely approximated by least squares linear regression. Although the net elastic force in the 2 element case still retains some nonlinearity, it too can be roughly approximated using least squares linear regression. Thus, the elastic force of a coupled DEG configuration can be modeling using a linear spring.

Similarly, the net capacitance and the net voltage can be calculated by summing the capacitances and voltages of DEG 1 and DEG 2. As for the energy terms, since the two DEG's are identical, the average energy loss per cycle due to electrical losses and mechanical losses will be doubled as will the energy harvested. Further analysis of the results of the coupled DEG simulation revealed that the energy harvested per cycle can be accurately simulated using a resistor, $R_{H}$, in parallel with the film similar to how the electrical losses are simulated. The difference being that $R_{H}$ is a function of the oscillation frequency whereas $R_{L}$ is frequency invariant. This discovery is important in the use of the mobility analogy in the next section.

The following graphs show the operation of two coupled 4 element DEG's by first showing individual operation and then the combined operation. The first three 
graphs show the displacement, voltage and force of each DEG separately but plotted on the same graphs which give visual representations of the phase shift between the two DEG's. The following six graphs show the summed voltage, capacitance and force of the coupled DEG's in both the time and displacement domains. The final graph plots the elastic force of each DEG, the net elastic force and the fit for the net elastic force which shows how two nonlinear DEG's can be coupled so that their resulting net elastic force in linear.
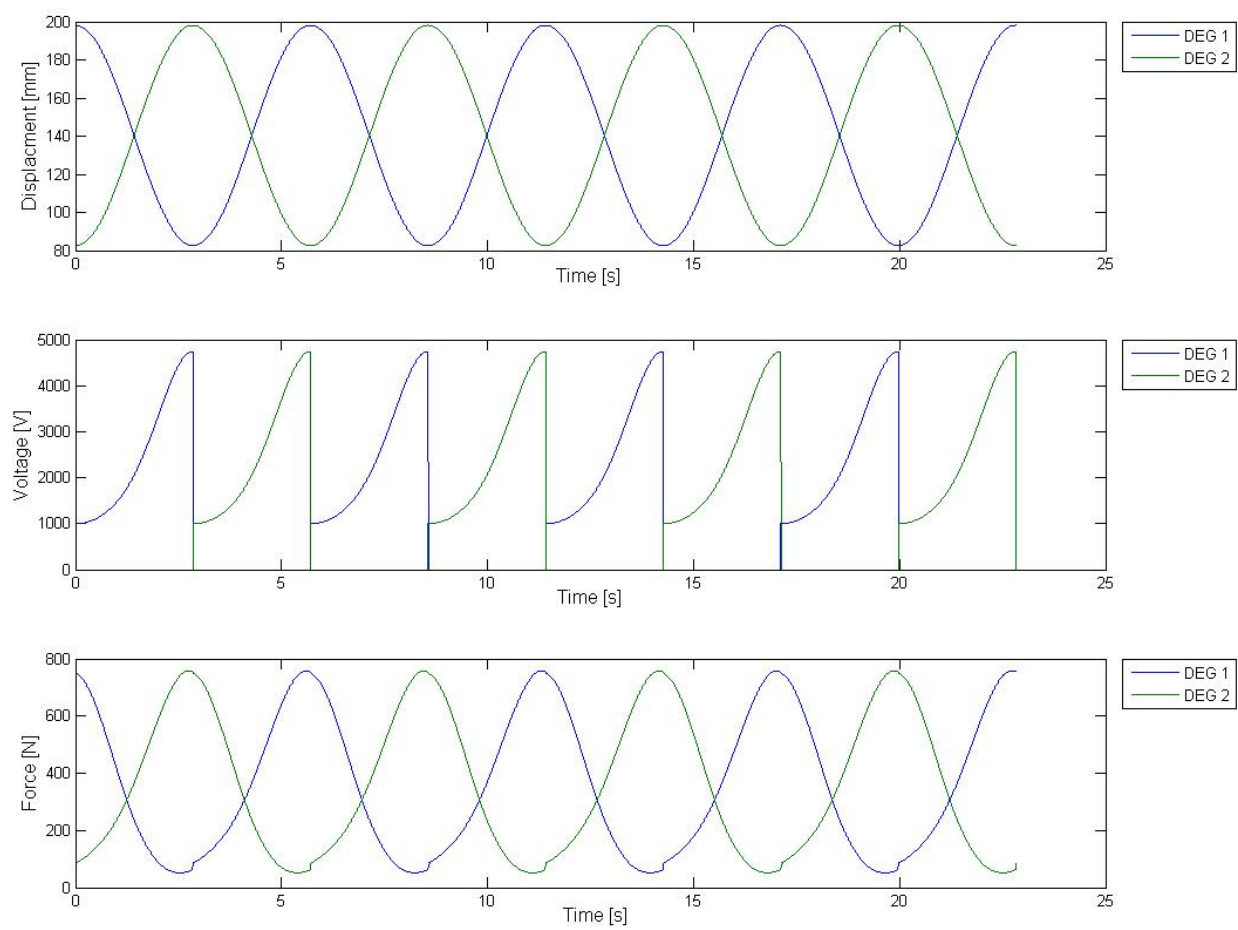

Figure AF - Displacement, voltage and force graphs in the time domain for two DEG's that are operating 180 degrees out of phase with each other. 

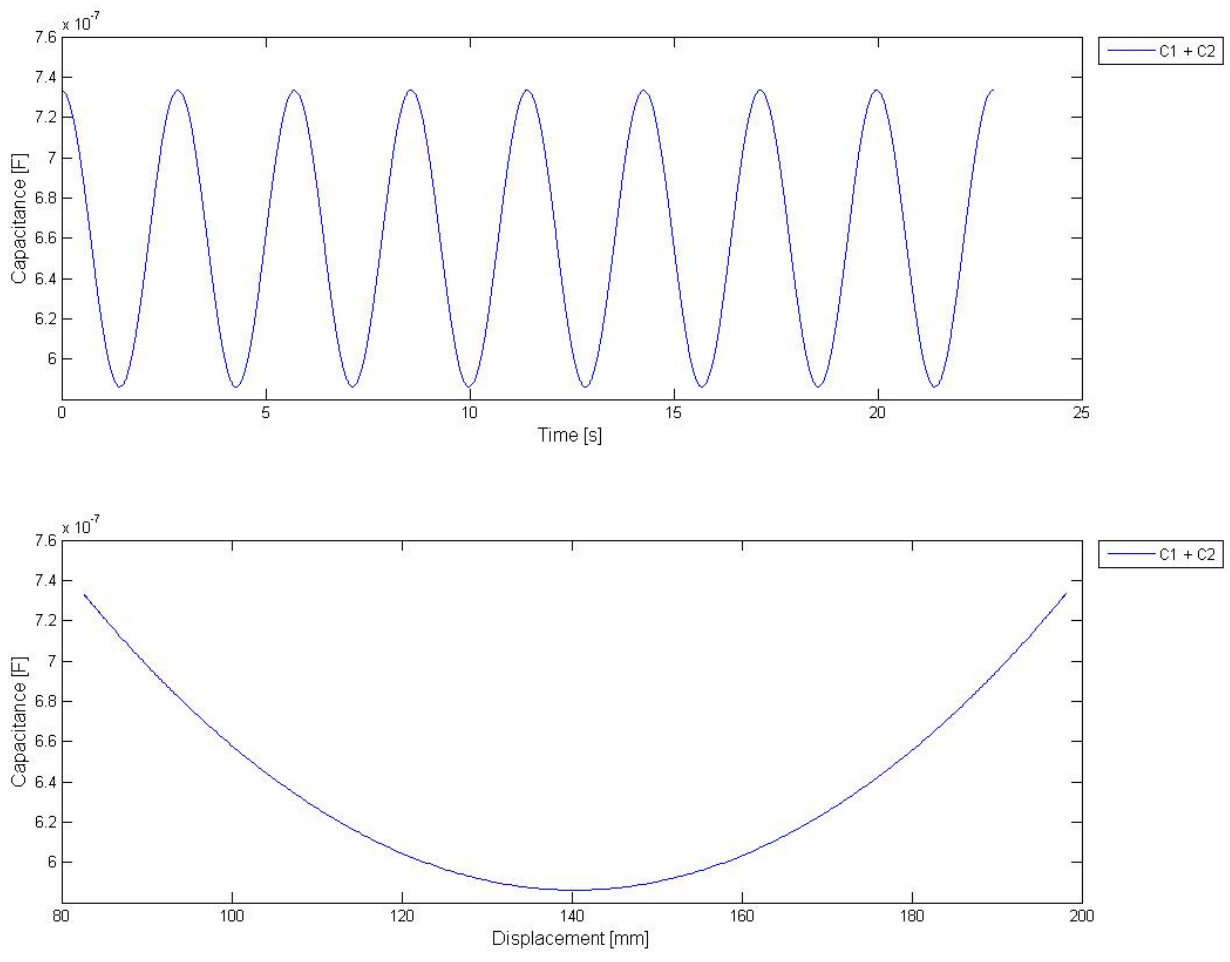

Figure AG - Capacitance in the time and displacement domains for the coupled DEG configuration. 

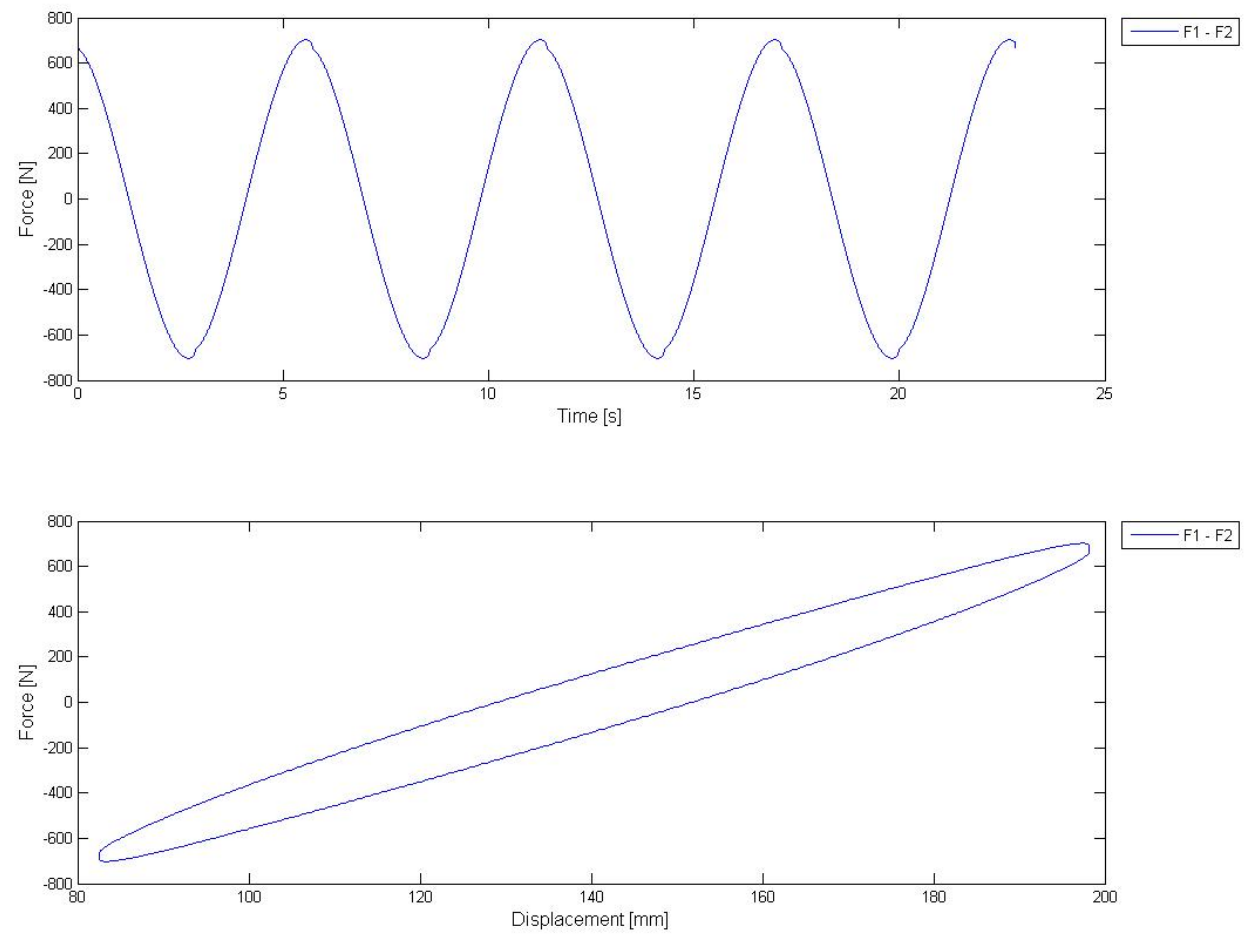

Figure AH - Force in the time and displacement domains for the coupled DEG configuration. 


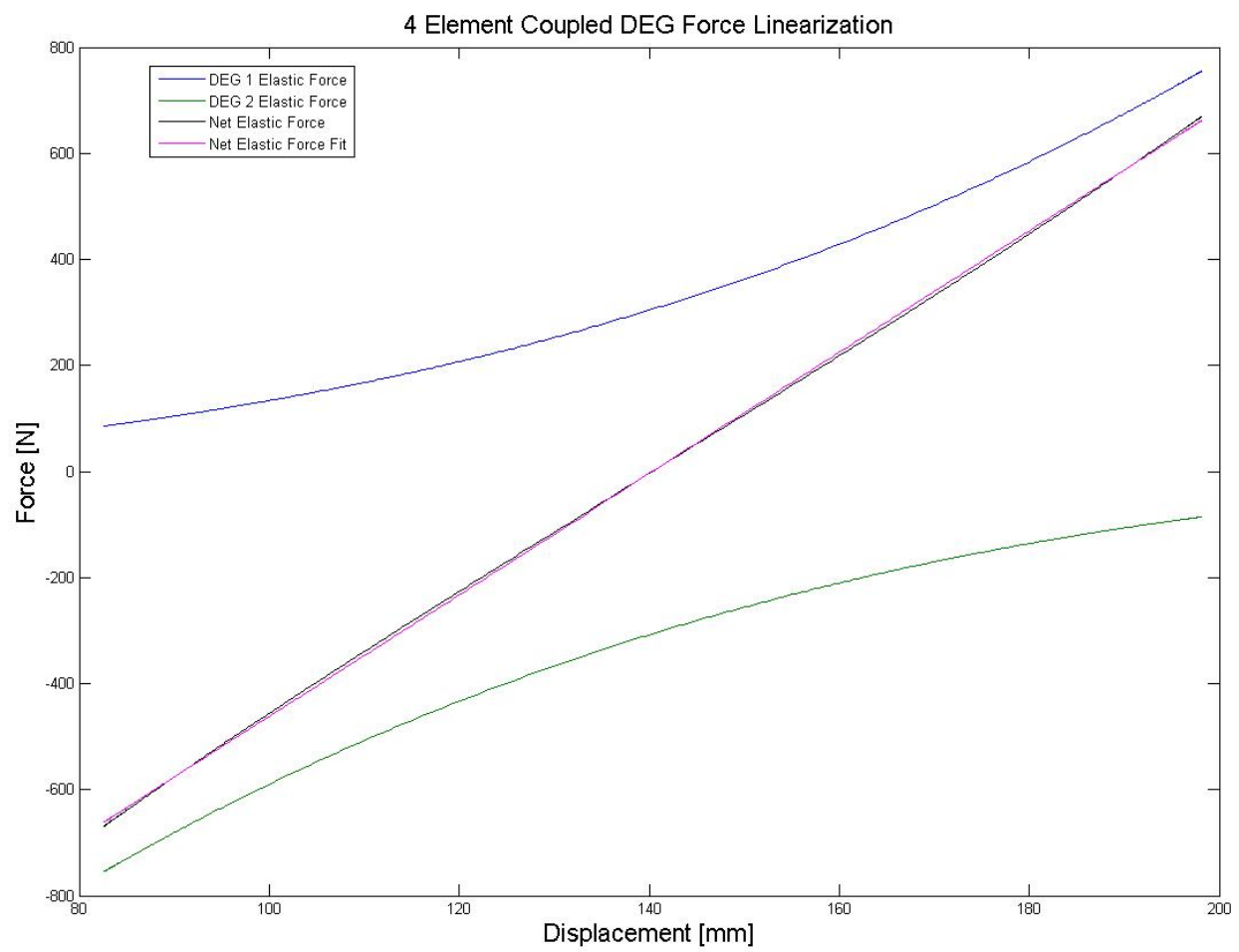

Figure AJ - This graph shows that the net force of two nonlinear DEG's results in a configuration that has a linear force. 

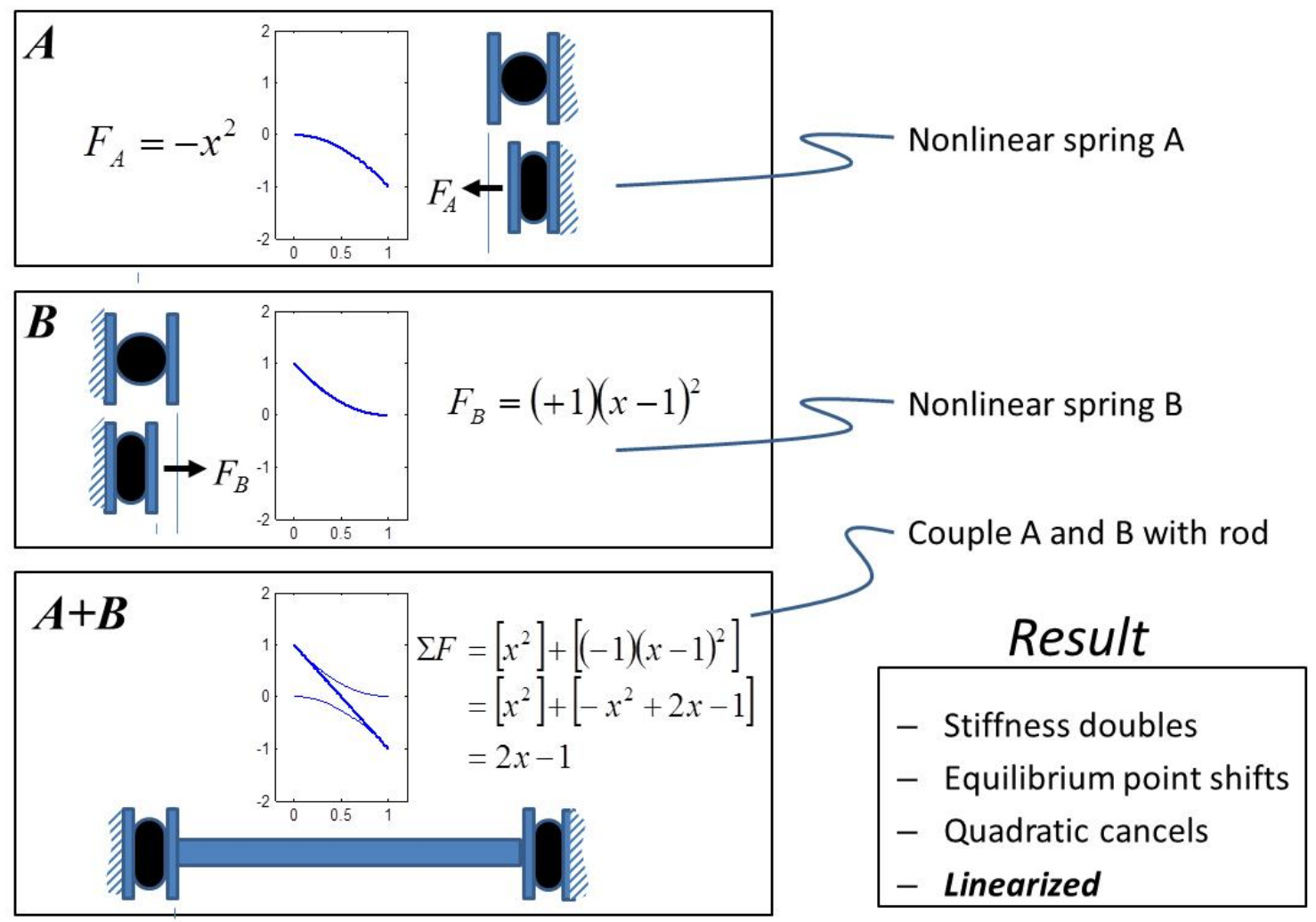

Figure AK -A diagram illustrates the technique used to linearize the generator spring rate. Opposing and coupling two matched quadratic spring elements (A) and (B) linearizes the spring rate $(A+B)$.

\section{DEG Modeling}

\section{Two-phase DEG Model and Best Fit Parameters:}

The previous two sections dealt with the details of the single DEG simulation and the coupled DEG simulation. However all of these details are not required when optimizing the DEG design. The operation of two coupled DEG's can be described using four characteristics: elastic force, mechanical losses, electrical losses and energy harvested. The next step is to find accurate methods of representing these characteristics. At present, the elastic force is represented by a spring, the mechanical losses are represented by viscous damping and the electrical losses and energy harvested are both represented by resistors. Although this representation can accurately simulate the coupled DEG scenario, it is not the most effective way of doing so. A simplification is accomplished using a mechanical to electrical equivalence method called the mobility analogy, also known as the inverse analogy. In this analogy, the force is proportional to the current and the velocity is proportional to the voltage. The analogy allows electrical circuit elements to be related to mechanical circuit elements and vice versa. The objective of using this analogy is to convert the four characteristics into mechanical representations. The elastic force and the mechanical losses are already represented mechanically, i.e., using a spring rate and a viscous damping term. It remains for the electrical losses and the energy harvested to be converted from their electrical representations as resistors to mechanical representations. The mechanical equivalent of a resistor is a dashpot (viscous damping) where the resistance $(R)$ is proportional to the 
mechanical responsiveness $(r)$.The damping term $(c)$ is equal to the inverse of the responsiveness.

$$
R \propto r=\frac{1}{c}
$$

To show this analogy, start with the equation for a resistor,

$$
V=I R
$$

Then apply the mobility analogy where $V \propto u, I \propto F$ and $R \propto r$ (assume, for the example, that the proportionality constants are 1) resulting in,

$$
u=F r
$$

When the previous equation is rearranged it is clear that the mechanical damping term is equal to the inverse of the mechanical responsiveness.

$$
F=\frac{u}{r} \text { therefore } c=\frac{1}{r}
$$

Theoretically, direct conversion between the electrical resistance and the mechanical damping term is possible if the proportionality constants in the voltagevelocity and current-force relations are known. However in the DEG system, these relations are arbitrarily set based on the input parameters. For example, the simulation allows for the velocity to remain the same while the seed voltage is changed, thus changing the proportionality relations. Therefore a more effective way of converting the electrical resistance to mechanical damping is to use the energy terms. In the second section the average electrical energy loss per cycle was calculated using the following equation,

$$
W_{E L}=\frac{\int V^{2} d t}{R_{E L} N}
$$

Which can be rewritten as,

$$
W_{E L}=\frac{V_{r m s}^{2}}{R_{E L} f}
$$

A similar equation can be defined for the energy harvested per cycle using the resistor, $R_{H}$,

$$
W_{H}=\frac{V_{r m s}^{2}}{R_{H} f}
$$

If the energy loss terms are known then the previous equations can be rearranged to calculate the equivalent resistances for electrical energy losses and energy harvested, 


$$
R_{E L}=\frac{V_{r m s}^{2}}{W_{E L} f} \text { and } R_{H}=\frac{V_{r m s}^{2}}{W_{H} f}
$$

Now apply the mobility analogy to get,

$$
r_{E L}=\frac{u_{r m s}^{2}}{W_{E L} f} \text { and } r_{H}=\frac{u_{r m s}^{2}}{W_{H} f}
$$

The damping term, $c_{E L}$, for the dashpot that represents electrical energy loss and the damping term, $c_{H}$, for the dashpot that represents energy harvesting are the inverses of $r_{E L}$ and $r_{H}$ respectively. This is summarized in the following table.

\begin{tabular}{|c|c|c|c|c|}
\hline Model & $\begin{array}{c}\text { Mechanical } \\
\text { Losses }\end{array}$ & Elastic Force & $\begin{array}{c}\text { Electrical } \\
\text { Losses }\end{array}$ & $\begin{array}{c}\text { Energy } \\
\text { Harvested }\end{array}$ \\
\hline $\begin{array}{c}\text { DEG } \\
\text { Simulation }\end{array}$ & $F=c_{M L} u$ & $F=k x$ & $W_{E L}$ & $W_{H}$ \\
\hline $\begin{array}{c}\text { Mechanical } \\
\text { Model }\end{array}$ & $c_{M L}$ & $k$ & $r_{E L}=\frac{u_{r m s}^{2}}{W_{E L} f}$ & $r_{H}=\frac{u_{r m s}^{2}}{W_{H} f}$ \\
& & $c_{E L}=\frac{1}{r_{E L}}$ & $c_{H}=\frac{1}{r_{H}}$ \\
\hline
\end{tabular}

Table 8 -Coupled DEG mechanical equivalents.

The conclusion being that the coupled DEG simulation can be models as a spring and three dashpots in a parallel mechanical circuit.

The following tables give the values that can be used to simulate the coupled DEG simulation using the mechanical equivalents.

Spring Rate, $k\left[\frac{N}{m}\right]$, voltage and frequency invariant:

\begin{tabular}{|c|c|}
\hline 2 Element & 4 Element \\
\hline$k=2516.2$ & $k=11268.3$ \\
\hline
\end{tabular}

Table 9 - Spring rate.

Mechanical damping term, $c_{M L}\left[\frac{N s}{m}\right]$, voltage invariant: 


\begin{tabular}{|c|c|}
\hline 2 Element & 4 Element \\
\hline$c_{M L}=3.58 u_{r m s}^{-1.39}$ & $c_{M L}=62.6 u_{r m s}^{-1.1}$ \\
\hline
\end{tabular}

Table 10 - Mechanical losses.

Electrical loss damping term, $c_{E L}\left[\frac{N s}{m}\right]$, frequency invariant:

\begin{tabular}{|c|c|c|}
\hline Voltage [V] & 2 Element & 4 Element \\
\hline 500 & $c_{E L}=0.866$ & $c_{E L}=1.338$ \\
\hline 1000 & $c_{E L}=5.234$ & $c_{E L}=12.188$ \\
\hline 1500 & $c_{E L}=19.23$ & $c_{E L}=48.192$ \\
\hline
\end{tabular}

Table 11 - Electrical losses.

Energy harvesting damping term, $c_{H}\left[\frac{N s}{m}\right]$ :

\begin{tabular}{|c|c|c|}
\hline Voltage [V] & 2 Element & 4 Element \\
\hline 500 & $c_{H}=3.65 f^{-1}$ & $c_{H}=8.76 f^{-1}$ \\
\hline 1000 & $c_{H}=14.64 f^{-1}$ & $c_{H}=34.57 f^{-1}$ \\
\hline 1500 & $c_{H}=32.16 f^{-1}$ & $c_{H}=75.26 f^{-1}$ \\
\hline
\end{tabular}

Table 12 - Energy harvested.

\section{Scaling Equations:}

The last item to discuss in the modeling of a coupled DEG scenario is how to scale the four defining characteristics with mechanical power. For example, if the optimization requires four times the power, how do the spring rate $(k)$ and damping terms $\left(c_{M L}, c_{E L}, c_{H}\right)$ change in response? For the coupled DEG system, the three parameters that influence the mechanical power are the force, stroke and frequency as shown below:

$$
P=F u=-2 \pi F f A \sin (2 \pi f t)
$$

Where $u$ is the velocity $\left[\frac{m}{s}\right], F$ is the force $[\mathrm{N}], f$ is the frequency [Hz], $t$ is the time [s], and $A$ is the oscillation amplitude [m] (the stroke is twice the amplitude). Changing one of these parameters and keeping the other two the same will results in a change in the power. The scaling equations give the relationship between a scaling in any one of the parameters and the resulting scaling in the power, spring rate and damping terms.

Determining the scaling equations requires the analysis of three cases. One case for when each of the parameters is scaled and the other two are held constant. Since the four characteristics of the coupled DEG's simulation are modeled by springs and dashpots, the following will develop the scaling equations for a generic spring and 
dashpot and then relate to the four characteristics afterwards. The equations for a spring and dashpot are,

$$
F=k x \rightarrow k=\frac{F}{x} \text { and } F=c v \rightarrow c=\frac{F}{v}
$$

Case 1: Scale the force by $B_{1}$ and use the same stroke and frequency:

$$
B_{1} P=\left(B_{1} F\right) u=-2 \pi\left(B_{1} F\right) f A \sin (2 \pi f t)
$$

If the force is scaled by $B_{1}$ then the spring rate and the damping term are similarly scaled as shown below,

$$
B_{1} k=\frac{B_{1} F}{x} \text { and } B_{1} c=\frac{B_{1} F}{u}
$$

Case 2: Scale the stroke by $B_{2}$ and use the same force and frequency.

$$
B_{2} P=F\left(B_{2} u\right)=-2 \pi F f\left(B_{2} A\right) \sin (2 \pi f t)
$$

If the stroke is scaled by $B_{2}$ then the spring rate and damping term are inversely scaled as shown below,

$$
\frac{k}{B_{2}}=\frac{F}{B_{2} x} \text { and } \frac{c}{B_{2}}=\frac{F}{B_{2} u}
$$

Case 3: Scale the frequency by $B_{3}$ and use the same force and stroke.

$$
B_{3} P=F\left(B_{3} u\right)=-2 \pi F\left(B_{3} f\right) A \sin \left[2 \pi\left(B_{3} \mathrm{f}\right) \mathrm{t}\right]
$$

If the frequency is scaled by $B_{3}$ then the damping term is inversely scaled as shown below,

$$
k=\frac{F}{x} \text { and } \frac{c}{B_{3}}=\frac{F}{B_{3} u}
$$

The conclusion is that if the force is scaled by $B_{1}$, the stroke is scaled by $B_{2}$ and the frequency is scaled by $B_{3}$ then the power, spring rate and damping term are scaled by the following,

$$
B_{1} B_{2} B_{3} P \text { and } \frac{B_{1} k}{B_{2}} \text { and } \frac{B_{1} c}{B_{2} B_{3}}
$$

These equations can now be related back to the four characteristics of a coupled DEG simulation. The scaling relations are given in the following table when the force is scaled by $B_{1}$, the stroke is scaled by $B_{2}$ and the frequency is scaled by $B_{3}$, 


\begin{tabular}{|c||c|c|c|c|c|}
\hline Scaling Relations & $\begin{array}{c}\text { Mechanical } \\
\text { Power }\end{array}$ & Spring Rate & $\begin{array}{c}\text { Mechanical } \\
\text { Damping }\end{array}$ & $\begin{array}{c}\text { Electrical } \\
\text { Losses }\end{array}$ & $\begin{array}{c}\text { Energy } \\
\text { Harvested }\end{array}$ \\
\hline \hline Representation & $P$ & $k$ & $c_{M L}$ & $c_{E L}$ & $c_{H}$ \\
\hline Scaling & $B_{1} B_{2} B_{3} P$ & $\frac{B_{1} k}{B_{2}}$ & $\frac{B_{1} c_{M L}}{B_{2} B_{3}}$ & $\frac{B_{1} c_{E L}}{B_{2} B_{3}}$ & $\frac{B_{1} c_{H}}{B_{2} B_{3}}$ \\
\hline
\end{tabular}

Table 13 - Scaling Relations.

\section{Combined System Modeling}

\section{Coupling a DEG to Hydrodynamic Model of Galloping Bluff Body:}

A hydrodynamic model of the system has been presented in the Computational Fluid Dynamics section. This model has been implemented in PSPICE and is shown in Figure AL. In addition, scaled model parameters for a DEG are also included in the model.

Referring to Figure AL, the mass of the body is represented by C1. The spring rate of the DEG is represented by the inductor L1. The lift force is determined by the water flow velocity (Vflow) and the body velocity (Vbody) and the nonlinear lift curve function.

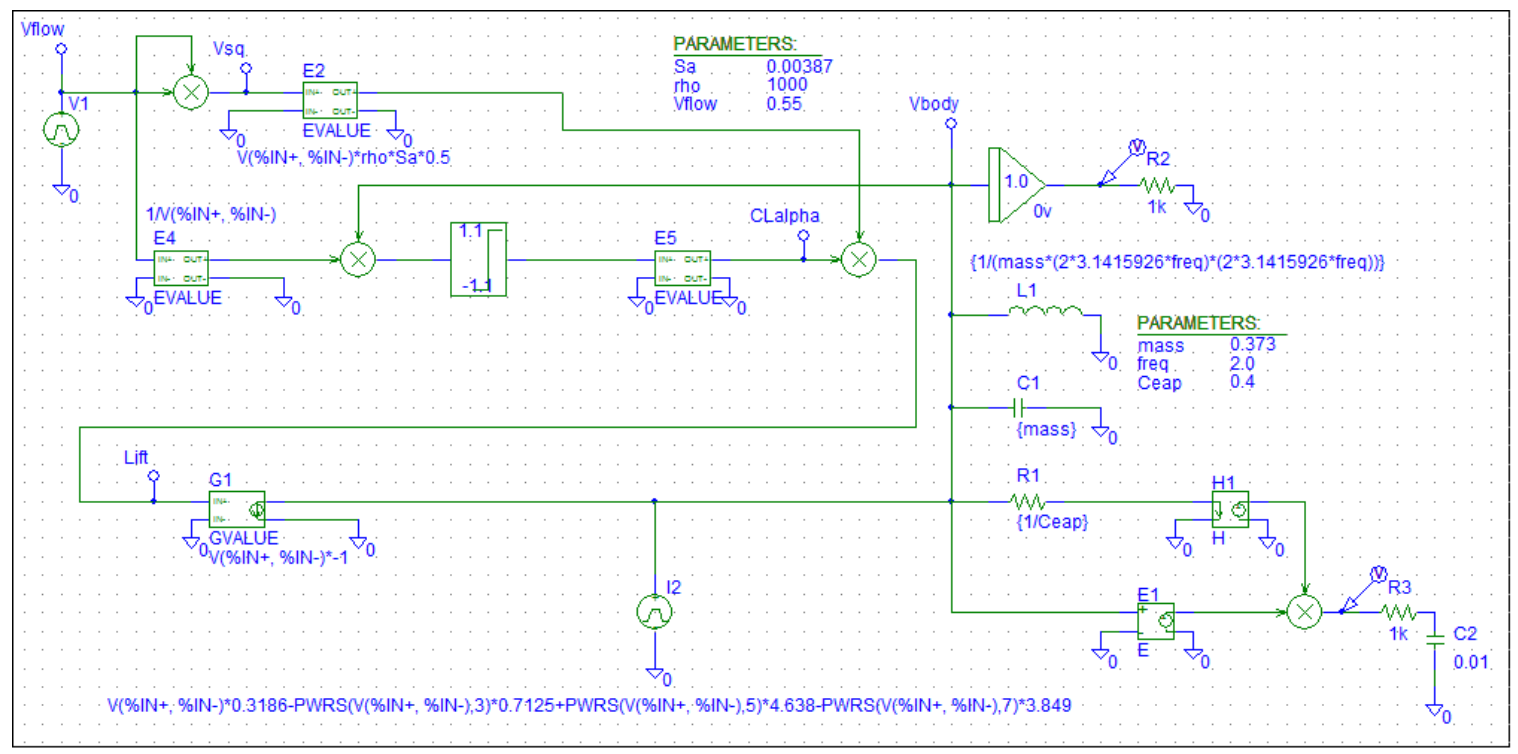

Figure AL - PSPICE model of the coupled system.

The first analysis case is using the system configuration presented in the Computational Fluid Dynamics section. The model substitutes a scaled DEG for the electromagnetic generator used in flume testing. In this case the model shows a similar displacement and 
mechanical power output of the system. Figure AM shows a simulated displacement and mechanical power production versus time.

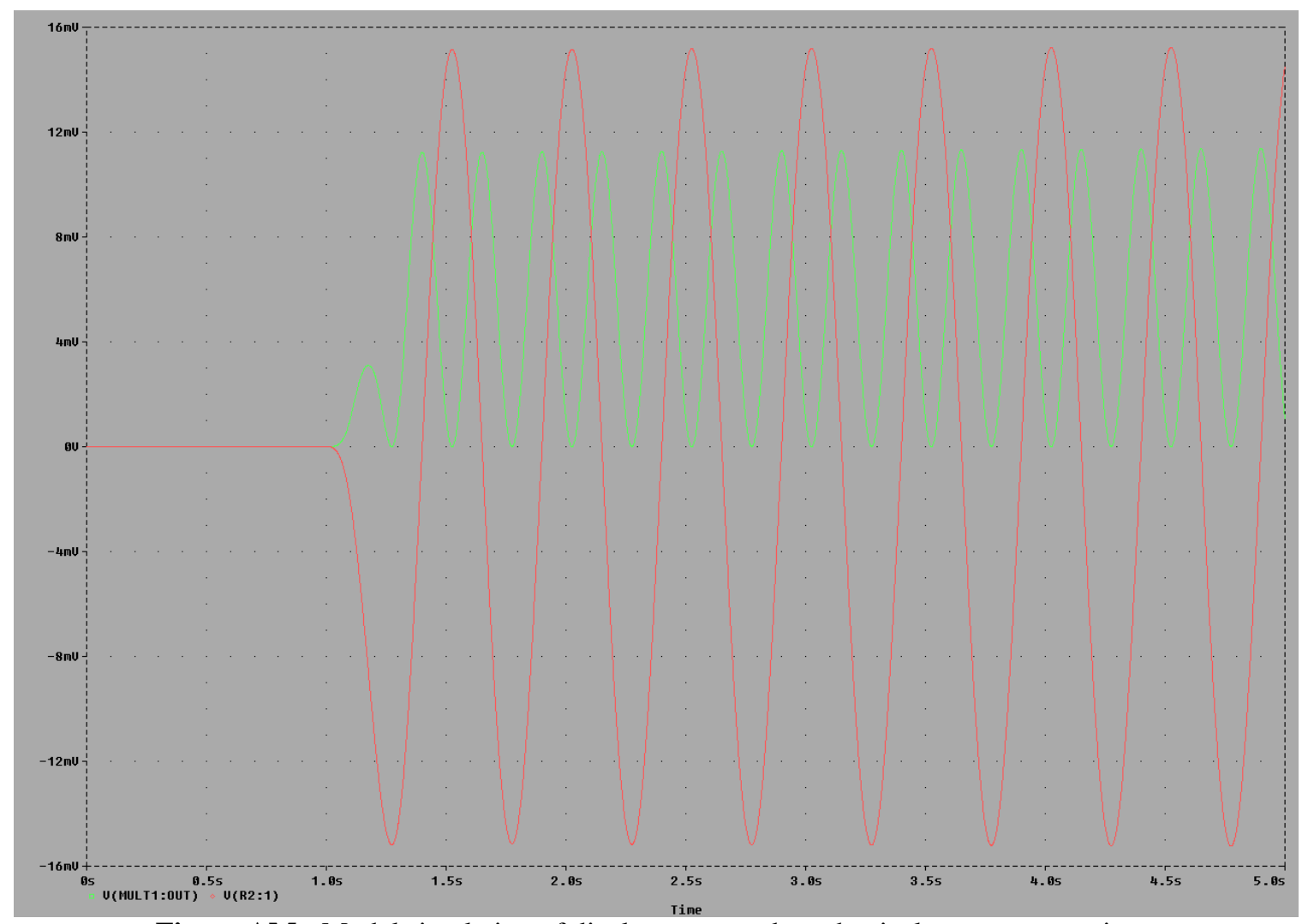

Figure AM - Model simulation of displacement and mechanical power versus time.

The model may also be used to show conditions of instability as demonstrated in Figure AN. Here, the mechanical load (and hence the DEG load) is changed to attempt to extract more mechanical power out of the system than shown above. The results are shown in figure below and indicate that if the mechanical load requires more power, the system fails to oscillate and is damped out. 


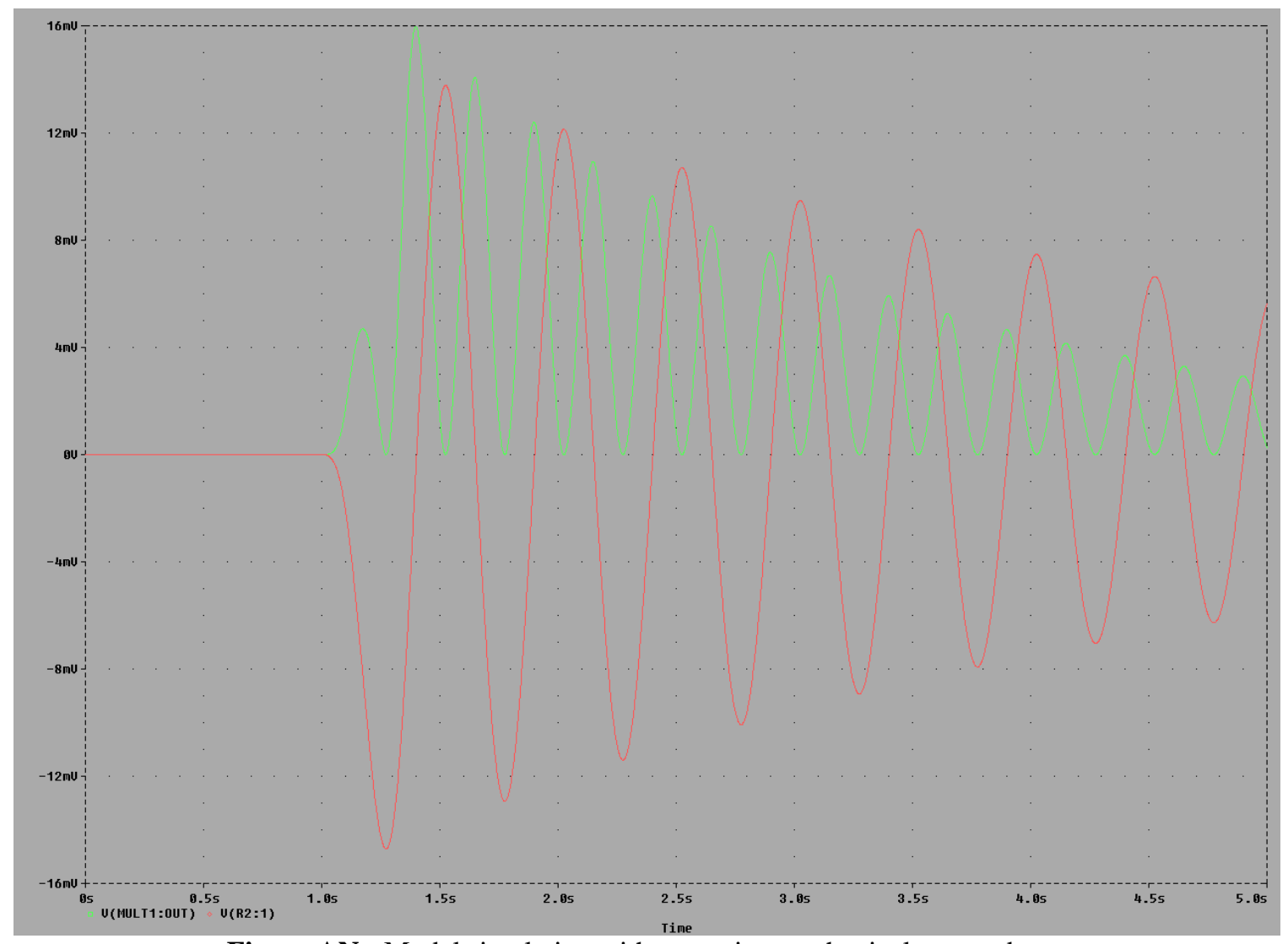

Figure AN - Model simulation with excessive mechanical power draw.

\section{Sizing a 1 Kilowatt Demonstration Unit:}

Using the models developed in the previous sections, extrapolation of model parameters can be made to scale up to a 1000 watt level design. At this point this scaling is meant to be instructive and not necessarily practical. Future experimental investigations will help guide practical scaling efforts while this model will help understand the interaction between different components of a complete system.

As an example, a 1000 watt system may be configured by using a GHEED with an area of 1.4 square meters and placed in a fast flowing river at 2 meters per second. The mass might be 28 kilograms and this model is shown in Figure AP. The DEG in this system is assumed to have been improved significantly and operates at approximately $60 \%$ efficiency. This requires a mechanical input power (from the GHEED) of 1667 watts to produce a rated output power of 1000 watts. For this simulation, the oscillation frequency is kept at 2 hertz but this parameter could change significantly in the future as this technology evolves. 


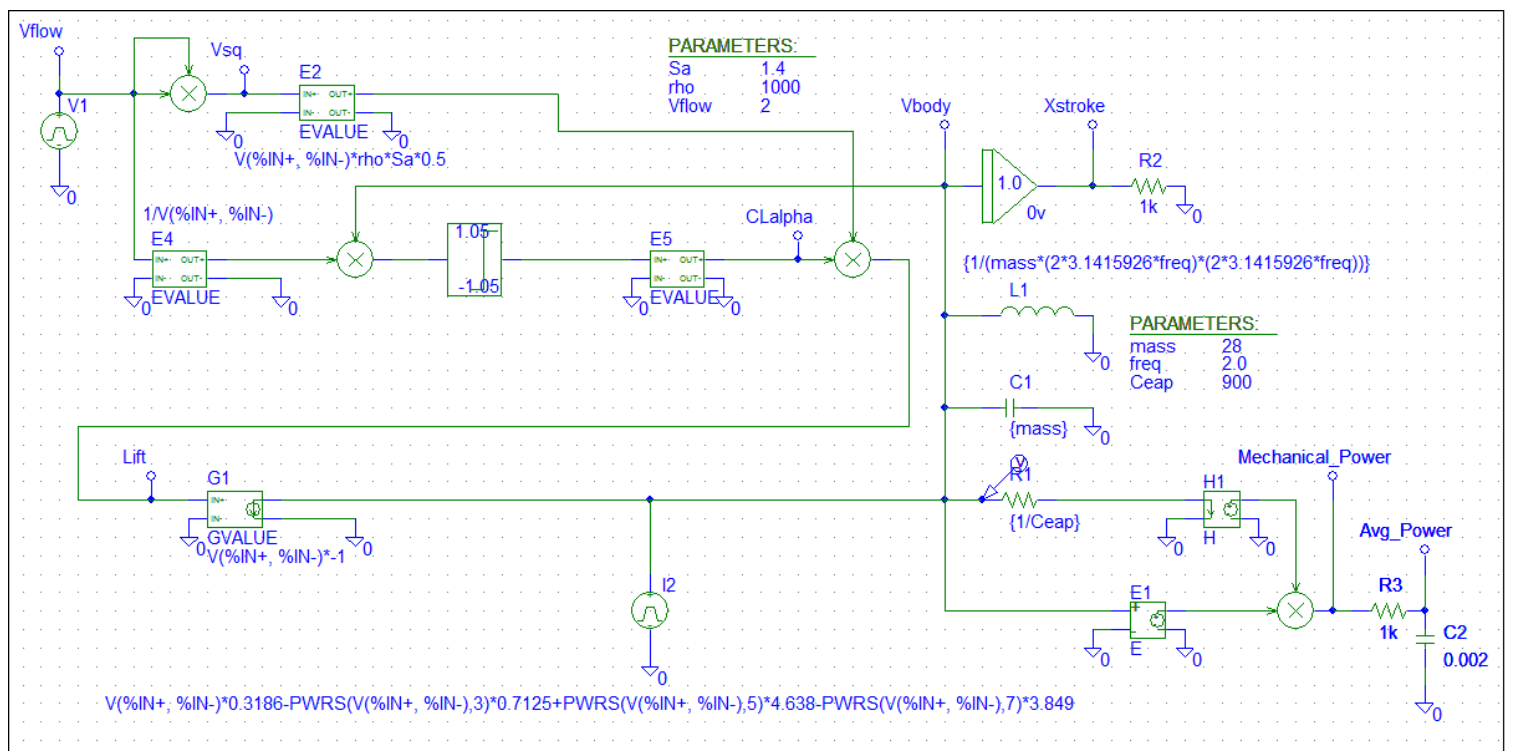

Figure AP - PSPICE model of a 1000 watt system.

Figure AQ shows a simulation of displacement and mechanical power versus time. The peak to peak stroke is approximately 0.35 meters and the average power generated is 1670 watts. The DEG would convert 1000 watts of the mechanical power into useable electrical power. Advances in DEG materials will be necessary to achieve these types of results. This model can be used to explore operational conditions that may arise in typical use by changing some of the variables in the model. 


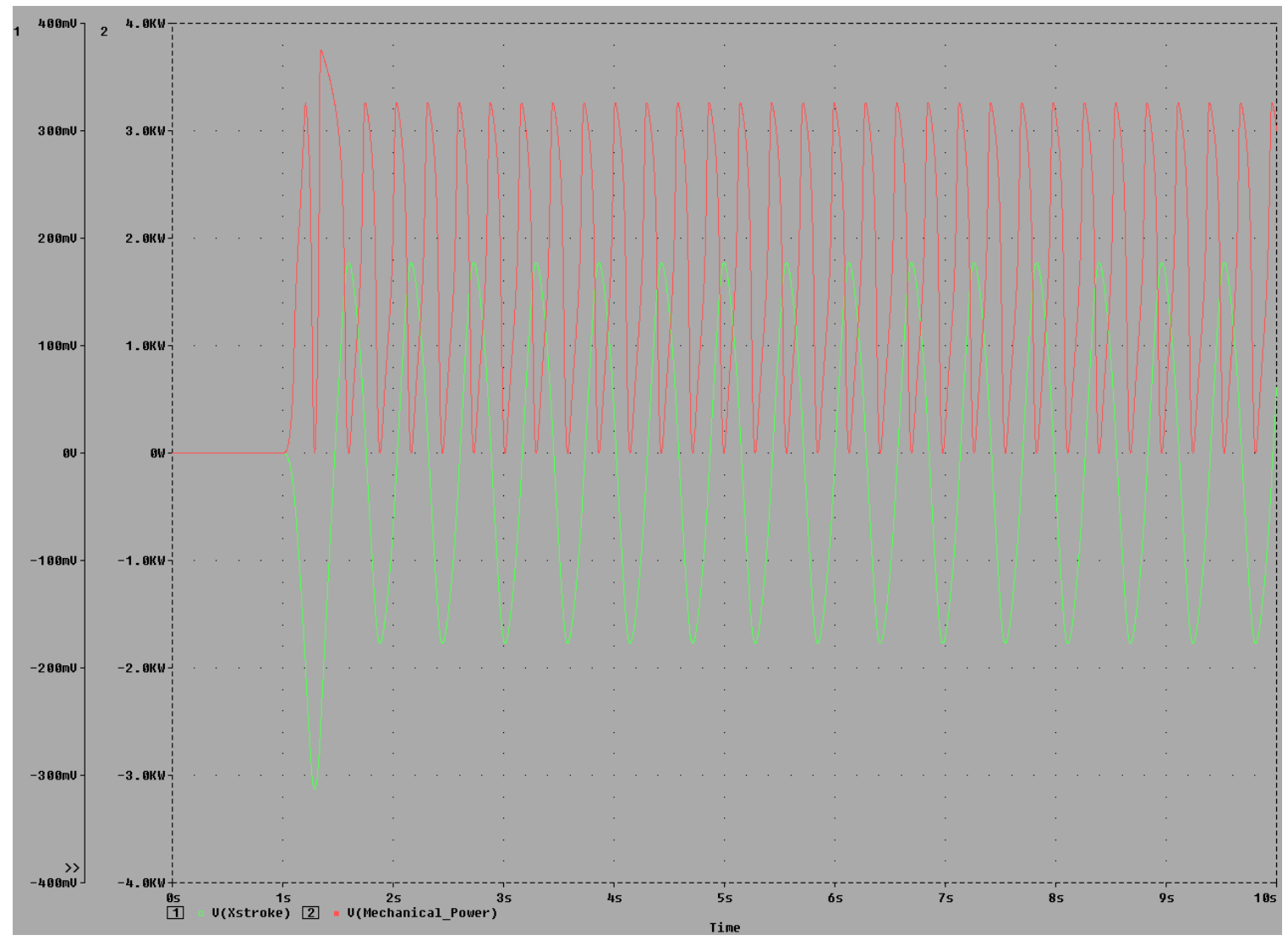

Figure AQ - Simulation results showing displacement and mechanical power versus time of a 1000 Watt system.

\section{LESSONS LEARNED}

The lessons learned in this program are best described from the perspective of future R\&D, which could also be described as, "What we would do next in light of what we now know." The future work envisioned for this overall concept is broken down into the key component areas associated with the GHEED:

\section{Galloping Body}

Application of limit cycle oscillations (gallop) was previously considered undesirable because of inherent instability for the purpose of extracting energy from unstable flows. In essence, the concept argues that stable mechanics, such as that of a turbine, should be employed in stable flows, while the mechanics of inherently unstable phenomena are best suited to inherently unstable flow regimes. During the recently concluded program it has been illustrated that a galloping device can be employed to reliably harvest electrical energy from an irregular flow. 


\section{Future Experiments:}

1. Field testing is clearly warranted. In the early months of the recently closed program, based purely on curiosity, an impromptu in-stream test was performed with the Gen-1 device (the impromptu test lasted only an hour or two, with the device being hand positioned in the stream). While this device worked quite well in the laboratory flume, it performed poorly in the real river environment. This prompted a design change toward a single pedestal that allows the free flow that is far more amenable to actual river conditions. But not yet characterized are fundamental in-service issues such as fowling, debris strikes, and flora/fauna interactions. For this a prototype will need to be installed for an extended performance assessment.

2. Testing so far shows that enough energy was extracted from a flow representing an untapped resource that there is reason to believe a device could be created with a desirable energy output. It is the team's opinion that this would be in the $1 \mathrm{KW}$ and upward range to be attractive for a commercial device which would be able to power a small radio or other similar device. The size and life expectancy of a device such as this are also areas that require further investigation and experiments. These can be very involved issues which were not explored due to the TRL of this project.

3. Other shapes not considered here may be of interest and could provide enhanced performance compared to the limited sampling utilized in this project and should be investigated.

\section{Other Structural Oscillation Modes:}

In the current research, a translational galloping motion was investigated. Other modes of oscillation might also be appropriate.

1. Torsion mode galloping: One mode might be a torsional mode. This may be more straightforward in terms of the fluid-structure interaction (hydrodynamics). The connection to an electrical generator may be simpler. The implications for impedance matching between the structural motion and the electrical generator may be better.

2.Flutter modes: Multi-mode flutter that couples plunge and twist motions may result in a limited cycle oscillation (LCO) that is more robust to changes in operating conditions. Typical flutter modes have an obvious cut-in speed, but this could be designed to be low enough to flutter at very low speeds. An advantage of multi-mode flutter is that there are more parameters to change including the relationship between an elastic axis, the center of gravity, and moments of inertia of a prism. As such, it may be easier to tune a device appropriately. Also, a device can be made that has an obvious hydrofoil shape, thus minimizing losses to turbulence and potentially generating more energy. 


\section{Shape Morphing}

Regarding shape morphing, the recently completed program clearly established that the few pre-existing data bases on the effects of varying the shape of a galloping control surface are generally not useful in this context - largely because the past studies have focused on steady flow conditions. As a result, an Edisonian approach will ultimately be required to down-select the ideal shape morphing strategy, and will likely evolve over an extended time frame. This data base should continue to evolve, but it also important that at any given moment in time those shapes which have already been studied can be interfaced in a way that optimizes performance, probably for a specific site application.

\section{$\underline{\text { DEG }}$}

A development pathway for Artificial Muscle is to provide dielectric elastomer generator elements for testing in GHEED prototypes. Artificial Muscle is currently developing tooling that makes self-reinforcing dielectric elastomer modules that are 10-fold larger than modules currently in mass production. The size, aspect ratio, and terminals of the modules are well-suited for use in prototype generators.

To extend the operating life of generators made from these modules, prior work on arrays of conductive polymer fuses should be further developed, and brought from bench-top to pilot scale. Electrode formulations optimized for interlayer adhesion and low resistivity are also desirable. Effort also needs to be directed toward development of electronics for robust and efficient energy conversion.

Application of DEGs for conversion of the mechanical energy into electrical energy, where the DEGs can be uniquely tuned to the dynamics of the mechanical system, and in addition because DEGs are inherently low frequency/large stroke, they are expected to be far better suited to these dynamics than age-old EMI strategies which favor very high frequency/small stroke. During the recently concluded program formation of a database has been initiated that is uniquely suited to integrating DEGs with GHEEDs.

1. (Mechanical) impedance matching of DEGs: The stiffness of the DEG is rather high. A more careful investigation of the required impedance between the mechanical and electrical sides of the device should be made. For small perturbations about some nominal LCO the action of the DEG makes it appear as a transformer. As such, it transforms impedances (mechanical to/from electrical) between the two energy domains. A proper investigation of this impedance matching should be made so that the DEG is designed to result in an LCO with desired characteristics (amplitude and frequency) and that the resulting (electrical) 
load has the desired characteristics. These two properties may be at odds with each other depending upon the LCO and electrical load.

2. Harvesting electronics: The electronics to harvest electricity need to be made and integrated with the GEH; that is, integrated with the structure in particular. How this device is controlled to switch as necessary needs to be designed. The specifics of how much energy must be transmitted to a particular load must be realized.

3. Other methods of transformation: The current research investigated DEGs as a primary focus and electro-magnetic generators in flume testing. While these two options are the most obvious, there may be other methods for electric generation that could be considered.

\section{Total System}

\section{Design \& Analysis:}

Additional research could be made into design rules and analysis techniques for galloping energy harvesters (GEHs). While the current effort investigated many of the techniques, a more formalized approach to GEH design could be made.

1.Development of design equations and scaling laws: A more formalized approach to GEH design should be made. This includes the necessary constitutive equations to describe the limit cycle oscillation (LCO) or gallop, the expected amplitude and power generated for a given design, and how power generation changes with operating condition. These relationships should be generalized and correlated with scaled models. Appropriate scaling laws should be made. Various higher order effects should be identified, and the regime identified where they are important to GEH performance.

2. Improved flume studies: The flume experiments made in this research were for a flume $\sim 6$ inches wide and $\sim 12$ inches deep. One difficulty with this setup is that wall, floor, and surface effects influence the galloping motion and other hydrodynamic measurements. A more thorough investigation of the performance of a GEH in a flume should be made. Such a study should take into account edge and other parasitic effects.

\section{Larger Prototype}

The practical question to be answered going forward is this - can the GHEED design eventually offer savings in magnets and maintenance that will more than offset any difference in efficiency? To begin answering this question, the next step is to design and build a reasonably-sized, bearing-free prototype, on the order of 500 Watt, optimized for simplicity.

A “large-scale” prototype energy harvester should be built and tested. Such testing could happen in a river or large flume. Even if a full-scale device cannot be made, one 
that is more representative of a real device in terms of aspect ratio, critical dimensions, and characteristic frequency should be tested.

\section{COST REDUCTION PATHWAYS}

At its inception this program proposed to advance the GHEED-DEG concept from TRL1 to TRL3. By its definition, it is premature to meaningfully discuss cost reduction pathways at TRL3; however, it is appropriate to identify which aspects of the technology are likely to drive overall cost. For instance, the varied properties required for development of shape morphing prisms can be accommodated with simple geometric strategies, and thus these components are not expected to dominate cost. In contrast, the generator is likely to be prominent among the costs of construction and implementation, and thus attention should be focused there. This can be achieved through direct inspection of the generator as well as through inspection of the interface. For the latter and per the above results section, it was found that varying the suspension stiffness of the GHEED had small if any effect on power generation, but could be used to reduce amplitude, and subsequently to reduce the required size of the generator.

Direct strategies for reducing generator costs should also be considered and begin with adopting dielectrics with lower hysteresis than acrylic. Reasonable candidate materials like silicone and polyurethane have lower hysteresis losses than acrylic, and therefore higher electromechanical efficiency. When less material is required to transact an electromechanical given amount of electric energy per cycle, cost can be reduced. Packaging can also be simplified, since there is less heat load to transfer out of the generator.

Robust, fault-tolerant dielectric elastomer cartridge constructions are another costreduction pathway. Fault-tolerant cartridges support operation at higher energy density energies that cause the occasional dielectric fault - enable more power to be transacted with less material, and therefore less expense .

Development of pilot-scale automatic manufacturing equipment and process is a third cost-reduction pathway. Single-layer cartridges hand-built on rapid-prototyped fixtures are the current state of the art in generator cartridges at Artificial Muscle, and are adequate for testing. Improved equipment and methods for pilot-scale manufacturing will lower the cost of producing working alpha-prototypes, and will refine estimates of dielectric generator costs achievable with volume production, steps part of the movement to Technology Readiness Levels 4 and 5. 


\section{CONCLUSION}

This project has developed a hydrokinetic energy generator that uses a novel energy collection system that is well suited to operation in fast moving streams and rivers. The energy-harvesting device is termed a Galloping Hydroelectric Energy Extraction Device (GHEED), which uses a galloping prism as a primer mover for energy generation and electric power. The GHEED combines a galloping device that converts the flow of water into an oscillating motion that will drive a dielectric elastomer generator (DEG). The DEG converts the mechanical motion of the oscillation into electricity. A phenomenon called galloping has been observed in electrical power lines in the presence of ice and/or rain. Wind blowing across ice-covered power lines creates lift causing the lines to oscillate. Galloping occurs when these oscillations increase to a sustained limit cycle where the oscillations are consistent and powerful. In this research, we have exploited these oscillations to create mechanical motion necessary to drive an electric generator. The galloping device relies on a limit-cycle oscillation that results from the nonlinear fluid-structure interaction between the water flow and the device. The galloping motion is induced by the water flow across the prism, and its effectiveness at different operating conditions depends upon the shape of the prism interacting with the flow. Controlling the shape of the prism can further enhance device performance. Ideally, the prism can be designed such that its shape changes with flow speed, ensuring that the proper conditions are maintained to encourage the galloping behavior.

The galloping oscillation of the device has been combined with a DEG, chosen because DEGs are well suited to converting rectilinear motion into electricity. For energy generation, the DEG acts as an elastic capacitor. It will be charged while in a strained state. Once charged, the linear motion of the oscillator will allow the DEG to relax, and excess energy is harvested from the DEG in its relaxed state. Finally, the motion of the oscillator once again strains the DEG and the cycle begins again.

This research effort investigated various aspects related to the device's operation and performance: device hydro-dynamics and mechanics, its characteristics and operation, prism shape change, the characteristics and design of a DEG generator, and the modeling, interaction, and integration of the galloping device and DEG generator together in a hydrokinetic energy harvesting system.

The program was divided into three separate research efforts with the goal of creating an integrated description of the final device useful for predicting both performance and cost.

1. Oscillator development and design

This effort was focused on characterizing the device's behavior analytically, numerically, and in a flume at the University of Pittsburgh. A non-linear, describing function model of the oscillatory behavior of the galloping device was made. The key information that is needed for this model is a description of the lift curve for the particular prism shape being investigated. Such lift curves could be measured in a flume, but the flume at the University of Pittsburgh spatially constrains the flow making such measurements difficult to achieve. An alternate method is numerical simulation using CFD (computational fluid dynamics). In an unfunded effort associated with this project, we investigated CFD simulations of various cross-sectional shapes including: a square prism and D-shaped prism for 
which hydrodynamic data exists in the literature, an equilateral triangle, and a trapezoidal prism. While this numerical route could potentially be used to find suitable shapes and tune known shapes for improved performance, the investigation was inconclusive regarding its expediency versus experimental investigations of a broad assortment of prism shapes.

Various shapes, including the square, D-shape, equilateral triangle, and trapezoid mentioned above were tested on a prototype, galloping device. The performance of the various shapes was compared for their ability to induce the limit-cycle oscillation and their ability to generate power. For the power generation experiments, an electro-mechanical generator was use as this was the most direct path to coupling mechanical and electrical domains. A DEG generator was not available for testing during this project.

Shape change of the prism was demonstrated. This shape change was induced by the water flow, and shows the potential to allow the device to change its shape automatically to suit the flow speed. Furthermore, the performance of the device was shown to improve slightly. It is possible for an optimized prism shape, consideration of the flow speeds experience in rivers, and proper design of the elastic characteristics of the prism itself that a GHEED device could be further optimized to enhance its power generation abilities.

2. DEG characterization and modeling

In this effort, DEGs were evaluated. Single DEGs were simulated, and these simulations were calibrated to data. DEGs exhibit non-linear behavior in both force and capacitance with displacement. Furthermore, the materials themselves exhibit losses, and these losses depend upon the RMS velocity (fortunately, they decrease with increasing RMS velocity). DEGs can be coupled to make a device with linear force-displacement curves, making their implementation in a GHEED straightforward, although material losses may still be significant. Numerical models of the single and coupled DEGs were developed.

\section{GHEED system modeling and integration}

The numerical models of the galloping device and the DEG were combined to generate a composite model of the GHEED. This model could be used to fully define the operating parameters and performance capabilities of a generator based on the GHEED design. The model was used to size a $1 \mathrm{~kW}$ demonstration unit. Additionally, this information could be used in the next phase of development, integrating the basic technological components for a TRL 4+ demonstration. Optimized working conditions for a GHEED device depend primarily on matching the impedance of the load to the impedance of the rest of the device, and this impedance match is a function of flow. Although not investigated as part of this effort, various impedance matching schemes could be used to achieve the proper impedance match.

At the start of the project the technology was TRL 1, the lowest level of technology readiness and scientific research was beginning translation to applied R\&D. The concept of a galloping energy harvester was conceived at the University of Pittsburgh, and 
theoretical analysis of the device had been performed. The objective of this research was to take the technology to TRL 3 by initiating active R\&D including further analytical studies and laboratory experiments to physically demonstrate and validate predictions of the separate elements of the technology. This goal has been achieved. A next step for the technology is progression to higher technology readiness levels.

\section{References}

1. $\{$ Hall $\}$ Hall, Douglas, et.al., Water Resources of the United States with an Emphasis on Low Head/Low Power Resources, Idaho National Engineering and Environmental Laboratory, April 2004

2. Leo L. Beranek, “Acoustics,” McGraw-Hill (1954).

3. \{Parkinson1964\} G. V. Parkinson, G. and J. D. Smith. The square prism as an aeroelastic non-linear oscillator. The Quarterly Journal of Mechanics and Applied Mathematics, 17(2):225-239, 1964.

4. \{Novak1972\} Milos Novak. Galloping oscillations of prismatic structures. Journal of the Engineering Mechanics Division, 98:27-46, 1972.

5. \{FLUTHY2010\} ANSYS. ANSYS Fluent Theory Guide, release 13.0 edition, November 2010.

6. Michael Kristufek, "On the use of computational fluid dynamics for evaluation of nonlinear hydrodynamic galloping energy harvester performance." M.S. Thesis, University of Pittsburgh, 2013.

\section{Acknowledgements}

The US Department of Energy for a grant (DE-EE0004571) to fund experiments related to River Devices to Recover Energy with Advanced Materials. The authors wish to acknowledge the contributions of the entire team:

Artificial Muscle Inc. (A Bayer MaterialScience Company) - Roger Hitchcock, James Biggs, Kurt Thompson (Intern)

University of Pittsburgh - Dr. Daniel Cole, Dr. Lisa Weiland, Michael Kristufek, Timothy Bagatti

Stanford Research Institute - Roy Kornbluh, Ron Pelrine, Brian McCoy

Bayer MaterialScience LLC - Daniel P. McMahon

\section{Disclaimer}

This report was prepared as an account of work sponsored by an agency of the United States Government. Neither the United States Government nor any agency thereof, nor any of their employees, makes any warranty, express or implied, or assumes any legal liability or responsibility for the accuracy, completeness, or usefulness of any information, apparatus, product, or process disclosed, or represents that 
its use would not infringe privately owned rights. Reference herein to any specific commercial product, process, or service by trade name, trademark, manufacturer, or otherwise does not necessarily constitute or imply its endorsement, recommendation, or favoring by the United States Government or any agency thereof. The views and opinions of authors expressed herein do not necessarily state or reflect those of the United States Government or any agency thereof. 